\title{
Composition of Commission 4
}

\section{Ephemerides/Ephémérides}

\author{
PRESIDENT: $\quad$ Chapront Jean \\ VICE-PRESIDENT: Krasinsky George A
}

\section{ORGANIZING COMMITTEE:}

Abalakin Victor $\mathrm{K}$

Arlot Jean-Eudes

Fukushima Toshio

He Miao-fu

\section{MEMBERS:}

Aoki Shinko

Bandyopadhyay A

Bangert John A

Bec-Borsenberger Annick

Bhatnagar Ashok Kumar

Bretagnon Pierre

Brumberg Victor A

Capitaine Nicole

Chapront-Touze Michelle

Chollet Fernand

Coma Juan Carlos

Davies Merton E

de Castro Angel

de Greiff J Arias

Deprit Andre

Di Xiaohua

Dickey Jean O'Brien

Duncombe Raynor L

Dunham David W

Eroshkin Georgiy I

Fiala Alan D

Fominov Alexandr $M$

Fu Yanning

Fursenko Margarita A

Glebova Nina I
Hohenkerk Catherine

Kaplan George H

Kinoshita Hiroshi

Lieske Jay $\mathrm{H}$

Gondolatsch Friedrich
Harper David
Henrard Jacques
Hilton James Lindsay
Ilyas Mohammad
Janiczek Paul M
Johnston Kenneth J
Klepczynski William J
Kolaczek Barbara
Kubo Yoshio
Lara Martin
Laskar Jacques
Lederle Trudpert
Lehmann Marek
Li Gi Man
Li Hyok Ho
Li Neng-Yao
Liu Bao-Lin
Lopez Moratalla Teodoro
Majid Abdul Bin A H
Morrison Leslie V
Mueller Ivan I
Newhall X X
Nguyen Mau Tung
O'Handley Douglas A

Schwan Heiner

Seidelmann P Kenneth

Standish E Myle

Pitjeva Elena

Reasenberg Robert D

Romero Perez M Pilar

Rossello Gaspar

Salazar Antonio

Shapiro Irwin I

Shiryaev Alexander A

Simon Jean-Louis

Sinzi Akira M

Sochilina Alla S

Soma Mitsuru

Thuillot William

Ting Yeou-Tswen

Tong $\mathrm{Fu}$

Van Flandern Tom

Wielen Roland

Wilkins George A

Williams Carol A

Williams James G

Winkler Gernot M R

Wytrzyszczak Iwona

Xian Ding-Zhang

Yallop Bernard D 


\section{Composition of Commission 5}

Documentation \& Astronomical Data/Documentation \& données astronomiques

PRESIDENT: $\quad$ Dluzhnevskaya Olga B

VICE-PRESIDENT: Genova Francoise

\section{ORGANIZING COMMITTEE:}

Bessell Michael S

Dickel Helene R

Hauck Bernard

Jenkner Helmut

Malkov Oleg Yu

\section{MEMBERS:}

A'Hearn Michael F

Abalakin Victor $\mathrm{K}$

Abt Helmut A

Aizenman Morris L

Alvarez Pedro

Andernach Heinz

Baker Norman $\mathrm{H}$

Benacchio Leopoldo

Benn Chris R

Bouska Jiri

Boyce Peter B

Cheung Cynthia Y

Chu Yaoquan

Cogan Bruce C

Coluzzi Regina

Corbin Brenda G

Creze Michel

Davis Morris S

Davis Robert J

de Boer Klaas Sjoerds

Dewhirst David W

Dixon Robert S

Dubois Pascal

Ducati Jorge Ricardo

Duncombe Raynor L

Egret Daniel

Eichhorn Guenther

Garstang Roy $\mathrm{H}$

Green David

Greisen Eric

Griffin Roger F

Grosbol Preben Johnson

Guibert Jean
Murtagh Fionn

Nakajima Koichi

Norris Raymond Paul

Ochsenbein Francois

Guo Hongfeng

Hanisch Robert J

Harvel Christopher Alvin

Hearn Anthony G

Heck Andre

Hefele Herbert

Heinrich Inge

Heintz Wulff D

Helou George

Huang Bi-kun

Kadla Zdenka I

Kalberla Peter

Kaplan George H

Kharin Arkadiy S

Kleczek Josip

Kuin Nicolaas Paulus M

Lantos Pierre

Lederle Trudpert

Lequeux James

Lesteven Soizick

Linde Peter

Liu Jinming

Lonsdale Carol J

Lortet Marie-Claire

Matz Steven Micheal

McLean Brian J

McNally Derek

Mcnamara Delbert $\mathrm{H}$

Mead Jaylee Montague

Meadows A Jack

Mein Pierre

Mermilliod Jean-Claude

Mitton Simon
Riegler Guenter R

Wells Donald C

Wielen Roland

Zhao Jun Liang

Nishimura Shiro Emer

Pamyatnykh Alexsey A

Pasian Fabio

Pasinetti Laura E

Paturel Georges

Pecker Jean-Claude

Pence William D

Philip A G Davis

Piskunov Anatoly E

Pizzichini Graziella

Polechova Pavla

Pucillo Mauro

Quintana Hernan

Raimond Ernst

Ratnatunga Kavan U

Remy Battiau Liliane G A

Renson P F M

Roman Nancy Grace

Rots Arnold H

Russo Guido

Sarasso Maria

Schilbach Elena

Schlesinger Barry $M$

Schlueter A

Schmadel Lutz D

Schmidt K H

Schmitz Marion

Schneider Jean

Serrano Alfonso

Shakeshaft John R

Shcherbina-Samojlova I

Sokolsky Andrej G

Spite Francois M 
Terashita Yoichi

Teuben Peter J

Tritton Susan Barbara

Tsvetkov Milcho K

Turner Kenneth $\mathrm{C}$
Uesugi Akira

Wallace Patrick T

Warren Jr Wayne $\mathrm{H}$

Weidemann Volker

Wenger Marc
Westerhout Gart

Wilkins George A

Wright Alan E

Zhao Yongheng 


\title{
Composition of Commission 6
}

\section{Astronomical Telegrams/Télégrammes astronomiques}

\author{
PRESIDENT: $\quad$ Marsden Brian G
}

VICE-PRESIDENT: Aksnes Kaare

\section{ORGANIZING COMMITTEE:}

Gilmore Alan C Nakano Syuichi

Green Daniel William E Roemer Elizabeth

\section{MEMBERS:}

Biraud Francois

Corbin Brenda G

Filippenko Alexei $\mathrm{V}$

Grindlay Jonathan E

Hers Jan
Isobe Syuzo

Kouveliotou Chryssa

Liu Jinming

Mattei Janet Akyuz

Nakamura Tsuko
West Richard M

Phillips Mark M

Pounds Kenneth A Tholen David J

Tsvetkov Milcho K

Yamaoka Hitoshi 


\section{Composition of Commission 7}

\section{Celestial Mechanics \& Dynamical Astronomy Mécanique céleste $\&$ astronomie dynamique}

$\begin{array}{ll}\text { PRESIDENT: } & \text { Hadjidemetriou John D } \\ \text { VICE-PRESIDENT: } & \text { Milani Andrea } \\ \text { SECRETARY: } & \text { Lemaitre Anne }\end{array}$

\section{ORGANIZING COMMITTEE:}

Burns Joseph A

Dvorak Rudolf

Froeschle Claude

Fukushima Toshio

\section{MEMBERS:}

Abad Alberto J

Abalakin Victor $\mathrm{K}$

Ahmed Mostafa Kamal

Akim Efraim L

Aksnes Kaare

Alexander Murray E

Anosova Joanna

Antonacopoulos Greg

Aoki Shinko

Balmino Georges G

Barberis Bruno

Barbosu Mihail

Batrakov Yuri V

Beauge Christian

Bec-Borsenberger Annick

Benest Daniel

Bettis Dale G

Beutler Gerhard

Bhatnagar K B

Bois Eric

Borderies Nicole

Boss Alan P

Bozis George

Branham Richard L

Breiter Slawomir

Bretagnon Pierre

Brieva Eduardo

Brookes Clive J

Broucke Roger

Brumberg Victor A

Brunini Adrian
Gerasimov Igor A

Heggie Douglas C

Knezevic Zoran

Muzzio Juan C

Calame Odile

Caranicolas Nicholas

Carpino Mario

Cefola Paul J

Chambers John E

Chapront Jean

Chapront-Touze Michelle

Chen Zhen

Choi Kyu-Hong

Cid Palacios Rafael

Colin Jacques

Contopoulos George

Cook Alan $\mathrm{H}$

Counselman Charles C

Cui Douxing

Danby J M Anthony

Davis Morris S

Deprit Andre

Descamps Pascal

Dikova Smiliana D

Dormand John Richard

Dourneau Gerard

Drozyner Andrzej

Duncombe Raynor L

Duriez Luc

Edelman Colette

El Bakkali Larbi

Elipe Sanchez Antonio

Emelianov Nikolaj V

Erdi B

Fernandez Silvia M
Sidlichovsky Milos

Soffel Michael

Sun Yisui

Ferraz-Mello Sylvio

Ferrer Martinez Sebastian

Fiala Alan D

Floria Luis

Fong Chugang

Galibina Irini $\mathrm{V}$

Galletto Dionigi

Gaposchkin Edward M

Gaska Stanislaw

Giacaglia Giorgio E

Giordano Claudia M

Giuliatti Winter Silvia M

Goldreich Peter

Gomes Rodney D S

Gonzalez Camacho Antonio

Goudas Constantine L

Grebenikov Evgenij A

Greenberg Richard

Groushinsky Nikolai P

Hallan Prem P

Hamid S El Din

Hanslmeier Arnold

He Miao-fu

Helali Yhya E

Henon Michel C

Henrard Jacques

Hori Genichiro

Hu Xiaogong

Huang Cheng

Huang Tianyi

Hut Piet 
Ivanova Violeta

Janiczek Paul M

Jefferys William $\mathrm{H}$

Journet Alain

Jovanovic Bozidar

Jupp Alan H

Kalvouridis Tilemahos

Kammeyer Peter C

Kholshevnikov Konstatin V

King-Hele Desmond G

Kinoshita Hiroshi

Klioner Sergei A

Klokocnik Jaroslav

Kovalevsky Jean

Kozai Yoshihide

Krasinsky George A

Krivov Alexander

Kuznetsov Eduard

Lala Petr

Laskar Jacques

Lazovic Jovan P

Lecavelier des Etangs Alain

Levine Stephen

Liao Xinhao

Lieske Jay H

Lin Douglas N C

Lissauer Jack J

Liu Wenzhong

Lu Ben ui

Lundquist Charles A

Ma Jingyuan

Maciejewski Andrzej J

Makhlouf Amar

Marchal Christian

Markellos Vassilis V

Marsden Brian G

Martinet Louis

Matas Vladimir R

Mavraganis A G

Meire Raphael

Melbourne William G

Merman G A

Message Philip J

Michel Patrick

Mignard Francois

Mikkola Seppo

Mioc Vasile

Morbidelli Alessandro

Mulholland John Derral
Musen Peter

Myachin Vladimir F

Nacozy Paul E

Nahon Fernand

Nobili Anna M

Noskov Boris N

Novoselov Victor $S$

O'Handley Douglas A

Ollongren A

Omarov Tuken B

Orellana Rosa Beatriz

Orus Juan J

Osorio Isabel Maria T V P

Osorio Jose J S P

Pal Arpad

Parv Bazil

Pauwels Thierry

Peale Stanton J

Petit Jean-Marc

Petrovskaya Margarita S

Pierce A Keith

Robinson William J

Rodriguez-Eillamil R

Roy Archie E

Ryabov Yu A

Sansaturio Maria E

Sato Massae

Scholl Hans

Schubart Joachim

Sconzo Pasquale

Segan Stevo

Sehnal Ladislav

Seidelmann P Kenneth

Sein-Echaluce M Luisa

Shapiro Irwin I

Sharaf Sh G

Sima Zdislav

Simo Charles

Simon Jean-Louis

Sinclair Andrew T

Skripnichenko Vladimir

Sokolov Viktor G

Sokolsky Andrej G

Sorokin Nikolai A

Souchay Jean

Standish E Myles

Stellmacher Irene

Steves Bonita Alice

Sultanov G F
Taborda Jose Rosa

Tatevyan S K

Tawadros Maher Jacoub

Taylor Donald Boggia

Thiry Yves R

Thuillot William

Tong Fu

Tremaine Scott Duncan

Tsuchida Masayoshi

Valsecchi Giovanni B

Valtonen Mauri J

Varvoglis $\mathrm{H}$

Vashkov'Yak Sof-Ya N

Vassiliev Nikolay $\mathrm{N}$

Veillet Christian

Vieira Martins Roberto

Vienne Alain

Vilhena Rodolpho Moraes R

Vondrak Jan

Walch Jean-Jacques

Walker Ian Walter

Watanabe Noriaki

Whipple Arthur L

Williams Carol A

Winter Othon Cabo

Wnuk Edwin

Wu Lianda

Wytrzyszczak Iwona

$\mathrm{Xia} \mathrm{Yi}$

$\mathrm{Xu}$ Jihong

$X u$ Pinxin

Yarov-Yarovoj M S

Yi Zhaohua

Yokoyama Tadashi

Yoshida Haruo

Yoshida Junzo

Yuasa Manabu

Zafiropoulos Basil

Zare Khalil

Zhang Sheng-Pan

Zhao Changyin

Zhdanov Valery

Zheng Jia-Qing

Zheng Xuetang

Zhou Hongnan

Zhou Ji-Lin

Zhu Wenyao 


\section{Composition of Commission 8}

Astrometry/Astrométrie

PRESIDENT: $\quad$ Jin Wen-Jing

VICE-PRESIDENT: Platais Imants K

\section{ORGANIZING COMMITTEE:}

Costa Edgardo

Ducourant Christine

Lattanzi Mario G

\section{MEMBERS:}

Abad Hiraldo Carlos

Andrei Alexandre $\mathrm{H}$

Andronova Anna A

Arenou Frederic

Argyle Robert William

Arias Elisa Felicitas

Bakhtigaraev Nail

Bakry Abdel-aziz

Ballabh Goswami Mohan

Bastian Ulrich

Belizon Fernando

Benedict George F

Benevides-Soares Pauolo

Bernstein Hans Heinrich

Bien Reinhold

Bougeard Mireille L

Branham Richard L

Bronnikova Nina $M$

Brosche Peter

Brouw W N

Bucciarelli Beatrice

Bunclark Peter Stephen

Carrasco Guillermo

Chen $\mathrm{Li}$

Chiumiento Giuseppe

Corbin Thomas Elbert

Creze Michel

Crifo Francoise

Cudworth Kyle McCabe

Dahn Conard Curtis

de Vegt Chr

Dejaiffe Rene J

Delmas Christian

Devyatkin Alexander V

Dick Steven J
Lu Chunlin

Roeser Siegfried

Schilbach Elena

Dick Wolfgang

Dommanget $J$

Duma Dmitrij P

Duncombe Raynor L

Einicke Ole H

Evans Dafydd Wyn

Fabricius Claus V

Fan $\mathrm{Yu}$

Feissel Martine

Firneis Maria G

Fomin Valery

Fomin Valery A

Franz Otto G

Fredrick Laurence W

Fresneau Alain

Froeschle Michel

Fujishita Mitsumi

Gatewood George

Gaume Ralph A

Gauss F Stephen

Geffert Michael

Gontcharov Gueorgui A

Gouda Naoteru

Goyal A N

Grudler Pierre

Guibert Jean

Guseva Irina S

Hajian Arsen R

Hanson Robert B

Hartkopf William I

Helmer Leif

Hemenway Paul D

Hering Roland

Heudier Jean-Louis

Hill Graham
Schwan Heiner

Stone Ronald Cecil

Turon Catherin

Hoeg Erik

Ianna Philip A

Ilin Alexei E

Irwin Michael John

Jackson Paul

Jahreiss Hartmut

Jefferys William $\mathrm{H}$

Johnston Kenneth $\mathbf{J}$

Jones Burton

Jones Derek H P

Jordi Carme

Journet Alain

Kanayev Ivan I

Kaplan George $\mathrm{H}$

Kharchenko Nina

Kharin Arkadiy S

Kislyuk Vitalij S

Klemola Arnold R

Klock B L

Kovalevsky Jean

Kurzynska Krystyna

Kuzmin Andrei V

Latypov A A

Le Poole Rudolf S

Lenhardt Helmut

Li Qi

Li Zhigang

Lindegren Lippincott Lennart

Lopez Carlos

Lopez Jose A

Lu Phillip K

Ma Wenzhang

MacConnell Darrell J

Marschall Laurence A

Mavridis L N 
McAlister Harold A

McLean Brian J

Mioc Vasile

Miyamoto Masanori

Molotaj Olexandr

Monet David G

Morbidelli Roberto

Muinos Haro Jose L

Murray C Andrew

Nakajima Koichi

Nefedeva Antonina I

Nikoloff Ivan

Noel Fernando

Nunez Jorge

Ohnishi Kouji

Oja Tarmo

Olsen Fogh H J

Osborn Wayne

Osorio Jose J S P

Pakvor Ivan

Pannunzio Renato

Pascu Dan

Pauwels Thierry

Perryman Michael A C

Pinigin Gennadij I

Polozhentsev Dimitrij D

Poma Angelo

Popescu Petre

Proverbio Edoardo

Pugliano Antonio

Rafferty Theodore J
Raimond Ernst

Reynolds John

Rizvanov Naufal G

Roemer Elizabeth

Russell Jane L

Sadzakov Sofija

Sanchez Manuel

Sanders Walt L

Santamaria Raffaele

Sarasso Maria

Sato Koichi

Schildknecht Thomas

Schmeidler $F$

Scholz Ralf Dieter

Schwekendiek Peter

Seidelmann P Kenneth

Shelus Peter J

Shen Kaixian

Shokin Yurij A

Soderhjelm Staffan

Solaric Nikola

Soma Mitsuru

Sovers Ojars J

Stavinschi Magdalena

Stein John William

Steinert Klaus Guenter

Stock Jurgen D

Teixeira Ramachrisna

Telnyuk-Adamchuk

Volodymyr

Tsujimoto Takuji
Upgren Arthur R

Urban Sean Eugene

Valbousquet Armand

Vallejo Miguel

van Altena William $F$

van Leeuwen Floor

Vass Gheorghe

Vertypolokh Olexander $Y$

Vilkki Erkki U

Volyanskaya Margarita Yu

Wallace Patrick T

Walter Hans G

Wan Lai

Wang Jiaji

Wang Zhengming

Wasserman Lawrence $\mathrm{H}$

Westerhout Gart

White Graeme Lindsay

Wielen Roland

Xia Yifei

$\mathrm{Xu}$ Jiayan

$\mathrm{Xu}$ Tong-Qi

Yan Haojian

Yang Tinggao

Yasuda Haruo

Yatsenko Anatolij I

Yatskiv Ya S

Ye Shuhua

Yoshizawa Masanori

Zacharias Norbert

Zhu Zi 


\section{Composition of Commission 9}

Instrumentation \& Techniques/Instrumentation \& Techniques

PRESIDENT: $\quad$ Su Ding-Qiang

VICE-PRESIDENT: Dennefeld Michel

\section{ORGANIZING COMMITTEE:}

Armstrong John Thomas

Brosch Noah

Cannon Russell D

Cui Xiangqun

Cullum Martin

Glass Ian Stewart

\section{MEMBERS:}

Abdul Aziz

Ahmad Zaharim

Ables Harold D

Ai Guoxiang

Aime Claude

Albrecht Rudolf

Alvarez Pedro

Andersen Torben E

Andreic Zeljko

Aparici Juan

Arnaud Jean-Paul

Arp Halton

Ashley Michael

Ashok N M

Assus Pierre

Atherton Paul David

Baba Naoshi

Babcock Horace W

Baffa Carlo

Bagrov Alexander

Ballesteros Ezequiel

Bao Keren

Baranne Andre

Barbieri Cesare

Barcia Alberto

Barden Samuel Charles

Barroso Jr Jair

Baruch John

Barwig Heinz

Baum William A

Becklin Eric E
Iye Masanori

Jacoby George $H$

Lawson Peter R

Lelievre Gerard

McLean Ian S

Nishimura Shiro Emer
O'Byrne John

Perryman Michael A C

Redfern Michael R

Saxena A K

Tango William J

Tsvetkov Milcho K
Beer Reinhard

Bensammar Slimane

Bhattacharyya J C

Bingham Richard G

Bonanno Alfio

Bonanno Giovanni

Bonnarel Francois

Bonneau Daniel

Borgnino Julien

Boyce Peter B

Brault James W

Breckinridge James B

Bridgeland Michael

Budiz-Joergensen Carl

Burton W Butler

Cao Changxin

Carter Bradley Darren

Chang Jin

Chelli Alain

Choudhary Debi Prasad

Christy James Walter

Citterio Oberto

Clarke David

Cohen Richard S

Cooke John Alan

Cornejo Alejandro A

Cosentino Rosario

Crawford David L

Cuevas Salvador C

Currie Douglas $\mathrm{G}$

Dall-Oglio Giorgio
Dan Xhixiang

Davis John

Denishchik Yurii

Desai Jyotindra N

Didkovsky Leonid

Diego Francisco

Dohlen Kjetil

Dokuchaeva Olga D

Douglas Nigel

Dravins Dainis

Dreher John W

Duchesne Maurice

Dudinov Volodymyr N

Dunkelman Lawrence

Edwin Roger P

Engels Dieter

Engvold Oddbjoern

Fabricant Daniel G

Fehrenbach Charles

Felenbok Paul

Fletcher J Murray

Fomenko Alexandr F

Ford Jr W Kent

Fort Bernard P

Foy Renaud

Fu Delian

Galan Maximino J

Gao Bilie

Gauss F Stephen

Gay Jean

Gibson David Michael 
Gillanders Gerard

Gillingham Peter

Gong Shou-shen

Gray Peter Murray

Griffiths Richard E

Grigorjev Victor M

Grosbol Preben Johnson

Guibert Jean

Gunji Shuichi

Gutcke Dietrich

Hadley Brian W

Hallam Kenneth L

Hammerschlag Robert $\mathrm{H}$

Hanisch Robert J

Hao Yunxiang

Harmer Charles F W

Harmer Dianne L

Heckathorn Harry $M$

Henden Arne Anthon

Heudier Jean-Louis

Hewitt Anthony V

Hilliard Ron

Hodapp Klaus-Werner

Honeycutt R Kent

Hough James

Hu Ning-Sheng

Huang Tieqin

Humphries Colin M

Hutter Donald John

Huygen Eric

Hysom Edmund J

Ilyas Mohammad

Irbah Abdanour

Ivchenko Vasily

Iyengar Srinivasan Rama

Jayarajan A P

Jeffers Stanley

Jenkner Helmut

Jiang Shengtao

Jiang Shi-Yang

Jones Barbara

Jordan Carole

Joseph Charles Lynn

Kaiser Mary E

Kalytis Romualdas

Karachentsev Igor D

Karpov Nikolai V

Kentischer Thomas

Kipper Tonu

Kissell Kenneth E
Klock B L

Klocok Lubomir

Koechlin Laurent

Koehler $\mathrm{H}$

Koehler Peter

Korovyakovskij Yurij P

Koshiishi Hideki

Kovachev B J

Kreidl Tobias J N

Kuehne Christoph F

Kulkarni Prabhakar V

Kuz'kov Vladimir P

Labeyrie Antoine

Laques Pierre

Lei Chengming

Lemaitre Gerard R

Li Depei

Li Ting

Li Xiaoqing

Li Xinnan

Li Zhigang

Lin Guanqing

Liu Zhong

Livingston William C

Lochman Jan

Lofdahl Mats $\mathrm{G}$

Louys Mireille Y

Lu Ruwei

Lynch David K

Mack Peter

Madau Piero

Mahra H S

Maillard Jean-Pierre

Major John

Malin David F

Malkamaeki Lauri J

Martins Donald Henry

Matsuura Shuji

Matz Steven Micheal

McGregor Peter John

McMullan Dennis

Megevand Denis

Meistas Edmundas $\mathrm{G}$

Meng Xinmin

Merkle Fritz

Mertz Lawrence N

Millikan Allan G

Minarovjech Milan

Morgan Brian Lealan

Morris Michael C
Morton Donald C

Murray Stephen S

Nakai Yoshihiro

Nakajima Tadashi

Nelson Jerry E

Newton Gavin

Niemi Aimo

Nishikawa Jun

Noumaru Junichi

O'Dell Charles R

Odgers Graham J

Ohtsubo Junji

O'Sullivan Creidhe

Owner-Petersen Mette M

Parker Neil

Pasian Fabio

Pati A K

Penny Alan John

Petford A David

Petrov Peter P

Picat Jean-Pierre

Povel Hanspeter

Pritchet Christopher J

Prokof'eva Valentina V

Pucillo Mauro

Qiu Puzhang

Queloz Didier

Racine Rene

Rakos Karl D

Ramsey Lawrence W

Reay Newrick K

Richardson Eric Harvey

Ring James

Robertson Norna

Robinson Lloyd B

Roddier Claude

Roddier Francois

Roellig Thomas L

Rosvick Joanne M

Rountree Janet

Ruder Hanns

Rupprecht Gero

Rusconi Luigia

Saha Swapan Kumar

Salez Morvan C

Sault Robert

Schneider Nicholas M

Schroeder Daniel J

Schultz Alfred Bernard

Schultz G V 
Schumann Joerg Dieter

Scuderi Salvatore

Sedmak Giorgio

Seifert Walter

Sergeev Aleksandr V

Servan Bernard

Shakhbazyan Yurij L

Shcheglov P V

Shen Changjun

Shen Parnan

Shi Shengcai

Shivanandan Kandiah

Shortridge Keith

Sim Elizabeth Mary

Skoda Petr

Slovak Mark Haines

Smyth Michael J

Snezhko Leonid I

Soltau Dirk

Steshenko N V

Storey John W V

Swings Jean-Pierre

Tapde Suresh Chandra

Title Alan Morton

Traub Wesley Arthur
Tueg Helmut

Tull Robert G

Tuthill Peter G

Ueno Munetaka

Ulich Bobby Lee

Vakili Farrokh

Valnicek Boris

Valtonen Mauri J

van Citters Gordon W

Velkov Kiril

Voges Wolfgang $\mathrm{H}$

Vrba Frederick J

Walker Alistair Robin

Walker David Douglas

Walker Gordon A H

Walker Merle F

Wallace Patrick T

Wampler E Joseph

Wang Chuanjin

Wang Lan-Juan

Wang Ya'nan

Wang Yiming

Wang Yong

Wang Zhengming

Ward Henry
Watson Frederick Garnett

Wei Wenren

Weiss Werner W

Welsh William $F$

West Richard M

Westphal James A

Windhorst Rogier A

Wlerick Gerard

Woehl Hubertus

Worden Simon P

Worswick Susan

Wu Linxiang

Wyller Arne A

Xiong Yoaheng

Yang Shijie

Yao Zhengqiu

Ye Binxun

Yin Xinhui

Zealey William J

Zhang Baozhou

Zhang Xiuzhong

Zhang Youyi

Zhao Zhaowang

Zheng Weimin

Zhu Nenghong 


\section{Composition of Commission 10}

Solar Activity/Activité solaire

PRESIDENT: Benz Arnold O

VICE-PRESIDENT: Melrose Donald B

\section{ORGANIZING COMMITTEE:}

Ai Guoxiang

Gopalswamy N

Harrison Richard A

Hood Alan

\section{MEMBERS:}

Abbasov Alik R

Abdelatif Toufik

Aboudarham Jean

Abramenko Valentina

Ahluwalia Harjit Singh

Alissandrakis $\mathrm{C}$

Almleaky Yasseen

Altrock Richard C

Altschuler Martin D

Aly Jean-Jacques

Amari Tahar

Ambastha A K

Ambroz Pavel

Anastasiadis Anastasios

Anderson Kinsey A

Antalova Anna

Antiochos Spiro Kosta

Antonucci Ester

Anzer Ulrich

Aschwanden Markus

Atac Tamer

Athay R Grant

Aurass Henry

Avignon Yvette

Babin Arthur

Babin Valerij G

Bagala Liria $G$

Bagare S P

Ballester Jose Luis

Baranovsky Edward A

Barrow Colin H

Batchelor David Allen

Beckers Jacques M

Bedding Timothy
Jackson Bernard V

Kozlovsky B Z

Poletto Giannina

Schrijver C J

Beebe Herbert A

Bell Barbara

Belvedere Gaetano

Benevolenskaya Elena

Berger Mitchell

Bergeron Jacqueline A

Berghmans David

Berrilli Francesco

Bhatnagar Arvind

Bocchia Romeo

Bogdan Thomas J

Bommier Veronique

Bondal Krishna Raj

Bornmann Patricia L

Bougeret Jean-Louis

Boyer Rene

Brajsa Roman

Brandenburg Axel

Brandt Peter N

Braun Douglas Clifford

Bray Robert J

Brekke Pal Ording lie

Bromage Barbara J I

Brooke John M

Brosius Jeffrey William

Brown John C

Browning Philippa

Bruner Marilyn E

Bruns Andrey V

Bruzek Anton

Buecher Alain

Buechner Joerg

Bumba Vaclav

Cadez Vladimir
Shibata Kazunari

Stepanian N N

van Driel Gesztzlyi L

Venkatakrishnan P

Cally Paul S

Cane Hilary Vivien

Cargill Peter J

Carlquist Per A

Cauzzi Gianna

Chambe Gilbert

Chandra Suresh

Chaoudhuri Arnab

Chapman Gary A

Charbonneau Paul

Chen Pengfei

Chen Zhencheng

Chernov Gennadij P

Chertok Ilia M

Chertoprud Vladim E

Chiuderi-Drago Franca Pr

Chiueh Tzihong

Choudhary Debi Prasad

Choudhuri Arnab Rai

Chupp Edward L

Cliver Edward W

Coffey Helen E

Collados Manuel

Conway Andrew J

Cook John W

Correia Emilia

Costa Joaquim E R

Coutrez Raymond A J

Covington Arthur E

Craig Ian Jonathan D

Cramer Neil F

Crannell Carol Jo

Culhane Leonard

Datlowe Dayton 


\begin{tabular}{|c|c|c|}
\hline Davila Joseph & Galsgaard Klaus & Holman Gordon D \\
\hline de Groot T & Gan Weiqun & Holzer Thomas Edward \\
\hline de Jager Cornelis & Garcia Howard A & Hong Hyon Ik \\
\hline Del Toro Iniesta Jose Carlos & Garcia Jose I de la rosa & Houdebine Eric \\
\hline Demoulin Pascal & Gary Gilmer Allen & Howard Robert F \\
\hline Deng Yuan-Yong & Gelfreikh Georgij B & Hoyng Peter \\
\hline Dennis Brian Roy & Gergely Tomas Esteban & Hudson Hugh S \\
\hline Dere Kenneth Paul & Ghizaru Mihai & Hughes David W \\
\hline Deubner Franz-Ludwig & Gibson David Michael & Hurford Gordon James \\
\hline Dezso Lorant & Gill Peter B J & Hyder C L \\
\hline Dialetis Dimitris & Gilliland Ronald Lynn & Ioshpa Boris $\mathrm{A}$ \\
\hline Ding Mingde & Gilman Peter A & Ireland Jack \\
\hline Ding Youji & Glatzmaier Gary A & Isliker Heinz \\
\hline Dinulescu Simona & Godoli Giovanni & Ivanchuk Victor I \\
\hline Dizer Muammer & Goedbloed Johan P & Ivanov Evgeny I \\
\hline Dollfus Audouin & Gokhale Moreshwar Hari & Ivchenko Vasily \\
\hline Dorch Soeren Bertil F & Gomez Daniel O & Jain Rajmal \\
\hline Dryer Murray & Gontikakis Constantin & Jakimiec Jerzy \\
\hline Dubau Jacques & Goossens Marcel & Jardine Moira Mary \\
\hline Dubois Marc A & Gopasyuk S I & Ji Haisheng \\
\hline Duldig Marcus Leslie & Grandpierre Attila & Jimenez Mancebo A j \\
\hline Dulk George A & Gray Norman & Jockers Klaus \\
\hline Dumitrache Cristana & Grib Sergey A & Jones Harrison Price \\
\hline Duncan Robert A & Gu Xiaoma & Jordan Stuart D \\
\hline Dunn Richard B & Guhathakurta Madhulika & Joselyn Jo Ann c \\
\hline Dwivedi Bhola Nath & Gurman Joseph B & Jovanovic Bozidar \\
\hline Dzubenko Nikolai & Gyori Lajos & Kaburaki Osamu \\
\hline Eddy John A & Hagen John P & Kahler Stephen W \\
\hline Efimenko Volodymyr M & Hagyard Mona June & Kalman Bela \\
\hline Elste Gunther H & Hammer Reiner & Kane Sharad R \\
\hline Emslie A Gordon & Hanaoka Yoichiro & Kang Jin Sok \\
\hline Engvold Oddbjoern & Hanasz Jan & Karlicky Marian \\
\hline Enome Shinzo & Hansen Richard T & Karpen Judith T \\
\hline Erdelyi Robert & Hanslmeier Arnold & Kaufmann Pierre \\
\hline Falchi Ambretta & Hara Hirohisa & Keppens Rony \\
\hline Falciani Roberto & Harvey John W & Kim Iraida $\mathrm{S}$ \\
\hline Fang Cheng & Hasan Saiyid Strajul & Kim Kap-sung \\
\hline Farnik Frantisek & Hathaway David H & Kiplinger Alan L \\
\hline Feibelman Walter A & Hayward John & Kitai Reizaburo \\
\hline Ferriz-Mas Antonio & Heinzel Petr & Kjeldseth-Moe Olav \\
\hline Fisher George Hewitt & Henoux Jean-Claude & Kleczek Josip \\
\hline Foing Bernard $\mathrm{H}$ & Herdiwijaya Dhani & Klein Karl Ludwig \\
\hline Fontenla Juan Manuel & Hermans Dirk & Kliem Bernhard \\
\hline Forbes Terry $\mathrm{G}$ & Hiei Eijiro & Klimchuk James A \\
\hline Fortini Teresa & Hildebrandt Joachim & Klvana Miroslav \\
\hline Fossat Eric & Hildner Ernest & Knoska Stefan \\
\hline Gabriel Alan H & Hirayama Tadashi & Kondrashova Nina $\mathrm{N}$ \\
\hline Gaizauskas Victor & Hoeksema Jon Todd & Kopecky Miloslav \\
\hline Galal A A & Hohenkerk Catherine & Kostik Roman I \\
\hline Galloway David & Hollweg Joseph V & Kotrc Pavel \\
\hline
\end{tabular}


Koutchmy Serge

Kovacs Agnes

Krivsky Ladislav

Krucker Sam

Krueger Albrecht

Kryshtal Alexander N

Kryvodubskyj Valery

Kubota Jun

Kucera Ales

Kuenzel Horst

Kuklin Georgly V

Kundu Mukul R

Kuperus Max

Kurochka Eugenia V

Kurochka L N

Kurokawa Hiroki

Landman Donald Alan

Lang Kenneth $\mathrm{R}$

Lantos Pierre

Lawrence John Keeler

Lazrek Mohamed

Leibacher John

Leroy Bernard

Leroy Jean-Louis

Li Chun-Sheng

Li Hui

Li Kejun

Li Son Jae

Li Wei

Lin Yuanzhang

Liritzis Ioannis

Liu Xinping

Livshits Mikhail A

Longbottom Aaron

Low Boon Chye

Lozitskij Vsevolod

Lundstedt Henrik

Luo Xianhan

Lustig Guenter

Machado Marcos

Mackay Duncan $\mathrm{H}$

MacKinnon Alexander L

MacQueen Robert M

Makarov Valentine I

Makita Mitsugu

Malherbe Jean-Marie

Malitson Harriet $\mathrm{H}$

Maltby Per

Malville J Mckim

Manabe Seiji
Mandrini Cristina Hemilse

Mann Gottfried

Maris Georgeta

Mariska John Thomas

Markova Eva

Martens Petrus C

Martres Marie-Josephe

Mason Glenn M

Masuda Satoshi

Matsuura Oscar T

Mattig W

Maxwell Alan

McCabe Marie K

McIntosh Patrick S

McKenna Lawlor Susan

McLean Donald J

Meerson Baruch

Mein Pierre

Mendes Da Costa Aracy

Messerotti Mauro

Mogilevskij Eh I

Mohan Anita

Moiseev I G

Moreno-Insertis Fernando

Moreton G E

Moriyama Fumio

Motta Santo

Muller Richard

Musatenko Sergij

Musielak Zdzislaw E

Nakagawa Yoshinari

Nakajima Hiroshi

Nakariakov Valery

Namba Osamu

Narain Udit

Neidig Donald F

Nelson Graham John

Neukirch Thomas

Neupert Werner M

Nishi Keizo

Nocera Luigi

Noens Jacques-Clair

Noyes Robert W

Nussbaumer Harry

Obridko Vladimir N

Oekten Adnan

Ofman Leon

Ohki Kenichiro

Ozguc Atila

Padmanabhan Janardhan
Paletou Frederic

Pallavicini Roberto

Palle Pere-Lluis

Palus Pavel

Pan Liande

Pap Judit

Parfinenko Leonid D

Park Young-Deuk

Parkinson John $\mathrm{H}$

Parkinson William H

Parnell Clare E

Pasachoff Jay M

Paterno Lucio

Pedersen Bent M

Peres Giovanni

Petrosian Vahe

Petrovay Kristof

Pevtsov Alexei A

Pflug Klaus

Phillips Kenneth J H

Pick Monique

Piddington Jack H Res Fel

Pneuman Gerald W

Poedts Stefaan

Pohjolainen Silja $\mathrm{H}$

Poland Arthur I

Poquerusse Michel

Porter Jason G

Preka-Papadema $\mathrm{P}$

Priest Eric R

Proctor Michael R E

Prokakis Theodore $\mathrm{J}$

Raadu Michael A

Rabin Douglas Mark

Rao A Pramesh

Raoult Antoinette

Rayrole Jean R

Rees David Elwyn

Reeves Edmond M

Reeves Hubert

Regulo Clara

Rieger Erich

Rijnbeek Richard

Robinson Jr Richard D

Roca Cortes Teodoro

Roemer Max

Romanchuk Pavel R

Rompolt Bogdan

Rosa Dragan

Roudier Thierry 
Rovira Marta Graciela

Roxburgh Ian W

Rozelot Jean-Pierre

Ruderman Michael S

Ruediger Guenther

Rusin Vojtech

Rust David M

Ruzdjak Vladimir

Ruzickova-Topolova B

Rybansky Milan

Ryutova Margarita $P$

Saemundson Thorsteinn

Sahal-Brechot Sylvie

Saito Kuniji

Sakao Taro

Sakurai Kunitomo

Sakurai Takashi

Sanchez Almeida Jorge

Saniga Metod

Sattarov Isroil

Sawyer Constance B

Schatten Kenneth $\mathrm{H}$

Schindler Karl

Schlueter A

Schmahl Edward J

Schmelz Joan T

Schmidt H U

Schmieder Brigitte

Schober Hans J

Schroeter Egon $\mathrm{H}$

Schuessler Manfred

Semel Meir

Shapley Alan H

Shea Margaret A

Sheeley Neil R

Shibasaki Kiyoto

Shine Richard A

Silberberg Rein

Simnett George M

Simon Guy

Sinha K

Smaldone Luigi Antonio

Smith Dean F

Smol-Kov Gennadij Ya

Sobotka Michal

Solanki Sami K

Soliman Mohamed Ahmed

Somov Boris V

Sotirovski Pascal

Spadaro Daniele
Spicer Daniel Shields

Spruit Henk C

Steiner Oskar

Stellmacher Goetz

Stenflo Jan O

Stepanov Alexander V

Steshenko N V

Stewart Ronald T

Stix Michael

Stoker Pieter H

Strong Keith T

Sturrock Peter A

Subramanian K R

Sukartadiredja Darsa

Sun Kai

Svestka Zdenek

Sykora Julius

Sylwester Barbara

Sylwester Janusz

Szalay Alex

Takakura Tatsuo Emer

Takano Toshiaki

Talon Raoul

Tamenaga Tatsuo

Tandberg-Hanssen Einar A

Tang Yuhua

Tapping Kenneth $\mathrm{F}$

Ternullo Maurizio

Teske Richard G

Thomas John H

Thomas Roger $\mathbf{J}$

Tifrea Emilia

Tlamicha Antonin

Tlatov Andrew $G$

Tobias Steven M

Tomczak Michal

Trellis Michel

Treumann Rudolf A

Tripathy Sushanta C

Tritakis Basil P

Trottet Gerard

Tsinganos Kanaris

Tsubaki Tokio

Tsubota Yukimasa

Tsuneta Saku

Tuominen Ilkka V

Uddin Wahab

Underwood James $\mathrm{H}$

Urpo Seppo I

Valnicek Boris van Allen James A

van den Oord Bert H J

van der Linden Ronald

van Hoven Gerard

van't-Veer Frans

Vaughan Arthur H

Veck Nicholas

Vekstein Gregory

Velkov Kiril

Velli Marco

Ventura Rita

Vergez Madeleine

Verheest Frank

Verma V K

Vial Jean-Claude

Vilmer Nicole

Vinluan Renato

Vinod S Krishan

Vitinskij Yurij I

Voitenko Yuriy M

Vrsnak Bojan

Walsh Robert

Wang Haimin

Wang Huaning

Wang Jia-Long

Wang Jingxiu

Wang Yi-ming

Webb David F

Wentzel Donat G

White Stephen Mark

Wiehr Eberhard

Wild John Paul

Wilson Peter R

Wittmann Axel D

Woehl Hubertus

Wolfson Richard

Woltjer Lodewijk

Wu De Jin

Wu Linxiang

Wu Mingchan

Wu Shi Tsan

Xu Aoao

Yan Yihua

Ye Shi-hui

Yeh Tyan

Yoshimura Hirokazu

You Jianqi

Yukhimuk Adam K

Yun Hong-Sik

Zachariadis Theodosios 
Zappala Rosario Aldo Zelenka Antoine

Zhang Heqi

Zharkova Valentina
Zhong Hongqi

Zhou Daoqi

Zhugzhda Yuzef D

Zirin Harold
Zlobec Paolo

Zou Yixin 


\section{Composition of Commission 12}

Solar Radiation \& Structure/Radiation \& structure solaires

PRESIDENT: $\quad$ Solanki Sami K

VICE-PRESIDENT: Bogdan Thomas J

\section{ORGANIZING COMMITTEE:}

Cauzzi Gianna

Christensen-Dalsgaard J

Cram Lawrence Edward

Dravins Dainis

\section{MEMBERS:}

Aboudarham Jean

Acton Loren W

Adam Madge $G$

Ai Guoxiang

Aime Claude

Alissandrakis C

Altrock Richard C

Altschuler Martin D

Ando Hiroyasu

Ansari S M Razaullah

Antia $\mathbf{H ~ M}$

Arnaud Jean-Paul

Artzner Guy

Athay R Grant

Ayres Thomas R

Baliunas Sallie L

Balthasar Horst

Barnes Graham

Beckers Jacques M

Beckman John E

Beebe Herbert A

Bendlin Cornelia

Benford Gregory

Bhatnagar Arvind

Bhattacharyya J C

Blackwell Donald E

Blamont Jacques-Emile

Bocchia Romeo

Boehm Karl-Heinz

Boehm-Vitense Erika

Bommier Veronique

Bonanno Alfio

Bonnet Roger $\mathrm{M}$

Book David L
Foukal Peter V

Gaizauskas Victor

Heinzel Petr

Kosovichev Alexander

Bornmann Patricia L

Bougeret Jean-Louis

Brandt Peter N

Brault James W

Bray Robert J

Breckinridge James B

Brosius Jeffrey William

Bruls Jo H

Bruner Marilyn E

Bruning David H

Bruzek Anton

Bumba Vaclav

Cadez Vladimir

Cavallini Fabio

Ceppatelli Guido

Chambe Gilbert

Chan Kwing Lam

Chapman Gary A

Chen Biao

Chertok Ilia M

Chistyakov Vladimir E

Clark Thomas Alan

Clette Frederic

Collados Manuel

Cook John W

Cox Arthur N

Craig Ian Jonathan D

Cramer Neil F

Dara Helen

de Jager Cornelis

Degenhardt Detlev

Del Toro Iniesta Jose Carlos

Delbouille Luc

Deliyannis Jean
Mariska John Thomas

Rovira Marta Graciela

Venkatakrishnan P

Wang Jingxiu

Demarque Pierre

Deming Leo Drake

Deubner Franz-Ludwig

Dezso Lorant

Ding Mingde

Diver Declan Andrew

Dogan Nadir

Dumont Simone

Dunkelman Lawrence

Dunn Richard B

Duvall Jr Thomas L

Dzubenko Nikolai

Ehgamberdiev Shurat

Einaudi Giorgio

Elliott Ian

Elste Gunther H

Epstein Gabriel Leo

Esser Ruth

Evans J V

Falciani Roberto

Fang Cheng

Feldman Uri

Fiala Alan D

Fisher George Hewitt

Fleck Bernhard

Fofi Massimo

Fomichev Valeri V

Fontenla Juan Manuel

Fossat Eric

Frazier Edward N

Froehlich Claus

Gabriel Alan H

Garcia Howard A

Garcia-Berro Enrique 
Gaur V P

Glatzmaier Gary A

Godoli Giovanni

Gokdogan Nuzhet

Goldman Martin V

Gomez Maria Theresa

Gopalswamy N

Gopasyuk S I

Gordon Charlotte

Grevesse Nicolas

$\mathrm{Gu}$ Xiaoma

Guhathakurta Madhulika

Hagyard Mona June

Hammer Reiner

Harvey John W

Hein Righini Giovanna

Hejna Ladislav

Hiei Eijiro

Hildner Ernest

Hill Frank

Hirayama Tadashi

Hoang Binh Dy

Holweger Hartmut

Hotinli Metin

House Lewis L

Howard Robert F

Hoyng Peter

Huang Guangli

Illing Rainer M E

Ivanov Evgeny I

Jabbar Sabeh Rhaman

Jackson Bernard V

Jefferies Stuart

Jones Harrison Price

Jordan Carole

Jordan Stuart D

Joshi G C

Kalkofen Wolfgang

Kalman Bela

Karlicky Marian

Karpen Judith T

Kato Shoji

Kaufmann Pierre

Kawaguchi Ichiro

Keil Stephen L

Khetsuriani Tsiala S

Kim Iraida S

Kim Yong-Cheol

Klein Karl Ludwig

Kneer Franz
Knoelker Michael

Kononovich Edward V

Kopecky Miloslav

Kostik Roman I

Kotov Valery

Kotrc Pavel

Koutchmy Serge

Krueger Albrecht

Kryvodubskyj Valery

Kubicela Aleksandar

Kucera Ales

Kuklin Georgly V

Kulcar Ladislav

Kundu Mukul R

Kuperus Max

Kurochka L N

La Bonte Barry James

Labs Dietrich

Landi Degl-Innocenti E

Landman Donald Alan

Landolfi Marco

Lantos Pierre

Lanzafame Alessandro C

Leibacher John

Leroy Jean-Louis

Li Linghuai

Lin Yuanzhang

Linsky Jeffrey L

Livingston William C

Locke Jack L

Lopez-Arroyo M

Luest Reimar

Lustig Guenter

Makarov Valentine I

Makita Mitsugu

Mandrini Cristina Hemilse

Marilli Ettore

Marmolino Ciro

Martinez Pillet Valentin

Mattig W

McKenna Lawlor Susan

Mein Pierre

Melrose Donald B

Mewe $\mathrm{R}$

Meyer Friedrich

Michard Raymond

Mihalas Dimitri

Milkey Robert W

Monteiro Mario J P F G

Moore Ronald L
Moreno-Insertis Fernando

Moriyama Fumio

Mouradian Zadig M

Muller Richard

Munro Richard H

Namba Osamu

Neckel Heinz

Nesis Anastasios

New Roger

Nicolas Kenneth Robert

Nishi Keizo

Nordlund Aake

Noyes Robert W

Oster Ludwig F

Owocki Stanley Peter

Padmanabhan Janardhan

Palle Pere-Lluis

Palus Pavel

Pande Mahesh Chandra

Papathanasoglou D

Parkinson William H

Pasachoff Jay M

Pecker Jean-Claude

Petrovay Kristof

Peyturaux Roger $\mathrm{H}$

Pflug Klaus

Phillips Kenneth J H

Pierce A Keith

Poquerusse Michel

Povel Hanspeter

Priest Eric R

Prokakis Theodore $\mathrm{J}$

Qu Zhong Quan

Rabin Douglas Mark

Radick Richard R

Raoult Antoinette

Rees David Elwyn

Reeves Edmond M

Regulo Clara

Roca Cortes Teodoro

Roddier Francois

Rodriguez Hildago Ines I

Roland Ginette

Roudier Thierry

Rusin Vojtech

Rutten Robert J

Rybansky Milan

Sakai Junichi

Sakurai Takashi

Samain Denys 
Sanchez Almeida Jorge

Saniga Metod

Sauval A Jacques

Schleicher Helmold

Schmahl Edward J

Schmidt Wolfgang

Schmieder Brigitte

Schmitt Dieter

Schober Hans J

Schuessler Manfred

Schwartz Steven Jay

Seaton Michael J

Semel Meir

Severino Giuseppe

Shallis Michael J

Shchukina Nataliya

Sheeley Neil R

Sheminova Valentina A

Shen Longxiang

Shine Richard A

Simon George W

Simon Guy

Singh Jagdev

Sinha K

Sivaraman K R

Skumanich Andre

Smith Peter L

Sotirovski Pascal
Souffrin Pierre B

Spicer Daniel Shields

Stathopoulou Maria

Staude Juergen

Stebbins Robin

Steffen Matthias

Steiner Oskar

Stenflo Jan O

Stix Michael

Suematsu Yoshinori

Svestka Zdenek

Swensson John W

Tandberg-Hanssen Einar A

Teplitskaya R B

Thomas John H

Torelli M

Tripathi B M

Trujillo Bueno Javier

Tsap T T

Tsiropoula Georgia

Tsubaki Tokio

Uchida Yutaka

Unno Wasaburo

Uus Undo

van Hoven Gerard

Vasileva Galina J

Vaughan Arthur H

Vial Jean-Claude
Vilmer Nicole

Vitinskij Yurij I

Volonte Sergio

von der Luehe Oskar

Vukicevic K M

Warwick James W

Weiss Nigel $O$

Wentzel Donat G

Wilson Peter R

Wittmann Axel D

Woehl Hubertus

Worden Simon P

Wu Hsin-Heng

Wu Linxiang

Wyller Arne A

Yoshimura Hirokazu

You Jianqi

Youssef Nahed $\mathrm{H}$

Yun Hong-Sik

Zampieri Luca

Zarro Dominic M

Zelenka Antoine

Zhou Daoqi

Zhugzhda Yuzef D

Zirin Harold

Zirker Jack B 


\section{Composition of Commission 14}

\section{Atomic \& Molecular Data/Données atomiques \& moléculaires}

PRESIDENT: $\quad$ Smith Peter L

VICE-PRESIDENT: Johansson Sveneric

\section{ORGANIZING COMMITTEE:}

Adelman Saul J

Berrington Keith Adrian

Biemont Emile

Feautrier Nicole

MEMBERS:

Aggarwal Kanti Mal

Allard Nicole

Allen Jr John E

Arduini-Malinovsky Monique

Artru Marie-Christine

Balanca Christian

Barnbaum Cecilia

Barrow Richard F

Bartaya R A

Bely-Dubau Francoise

Black John Harry

Boechat-Roberty Heloisa M

Bommier Veronique

Borysow Aleksandra

Branscomb L M

Brault James W

Bromage Gordon E

Burgess Alan

Carbon Duane F

Carroll P Kevin

Carver John $\mathrm{H}$

Chance Kelly V

Cook Alan $\mathrm{H}$

Corliss $\mathrm{C} \mathrm{H}$

Cornille Marguerite

Czyzak Stanley J

Dalgarno Alexander

Davis Sumner P

de Frees Douglas J

Delsemme Armand $\mathrm{H}$

Desesquelles Jean

d'Hendecourt Louis

Diercksen Geerd H F

Dimitrijevic Milan
Federman Steven Robert

Grevesse Nicolas

Martin William C

Mickelson Michael E

Dubau Jacques

Dufay Maurice

Dulieu Francois

Eidelsberg Michele

Epstein Gabriel Leo

Faucher Paul

Federici Luciana

Fillion Jean-Hugues

Fink Uwe

Flower David R

Fuhr Jeffrey Robert

Gabriel Alan H

Gallagher III John S

Gargaud Muriel

Garstang Roy H

Garton W R S

Glagolevskij Juri V

Goldbach Claudine

Grant Ian P

Heddle Douglas W O

Herold Heinz

Hesser James E

Hoang Binh Dy

House Lewis L

Huber Martin C E

Huebner Walter F

Iliev Ilian

Irwin Alan W

Jamar Claude A J

Johnson Donald R

Johnson Fred $M$

Joly Francois

Jordan Carole

Jordan H L Direktor
Rostas Francois

Ryabchikova Tanya

Stehle Chantal

Jorgensen Henning E

Jorgensen Uffe Graae

Kato Takako

Kennedy Eugene T

Kielkopf John F

Kim Zong Dok

Kingston Arthur E

Kipper Tonu

Kirby Kate $\mathbf{P}$

Kohl John L

Kroto Harold

Kurucz Robert L

Lambert David L

Landman Donald Alan

Lang James

Langhoff Stephenie Robert

Launay Francoise

Launay Jean-Michel

Lawrence G M

Layzer David

Le Bourlot Jacques

Le Floch Andre

Leach Sydney

Leger Alain

Lemaire Jean-louis

Lesage Alain

Loulergue Michelle

Lovas Francis John

Lutz Barry L

Maillard Jean-Pierre

Mason Helen E

McWhirter R W Peter

Mewe R

Mumma Michael Jon 
Nave Gilliam

Newsom Gerald $\mathbf{H}$

Nicholls Ralph W

Nollez Gerard

Nussbaumer Harry

Obi Shinya

O'Brian Thomas R

Oetken L

Oka Takeshi

Omont Alain

Orton Glenn S

Ozeki Hiroyuki

Palmeri Patrick

Parkinson William $\mathrm{H}$

Peach Gillian

Pei Chunchuan

Petrini Daniel

Petropoulos Basil Ch

Pettini Marco

Pfennig Hans $\mathrm{H}$

Phillips John G

Piacentini Ruben

Pradhan Anil

Querci Francois R

Quinet Pascal
Richter Johannes

Rogers Forrest J

Roncin Jean-Yves

Ross John E R

Roueff Evelyne M A

Ruder Hanns

Rudzikas Zenonas B

Sahal-Brechot Sylvie

Savanov Igor $\mathrm{S}$

Schrijver Johannes

Seaton Michael J

Sharp Christopher

Shore Bruce W

Sinha K

Smith Wm Hayden

Somerville William B

Sorensen Gunnar

Spielfiedel Annie

Stark Glen

Steenman-Clark Lois

Strachan Leonard Jr

Strelnitski Vladimir

Summers Hugh $P$

Swings Jean-Pierre

Takayanagi Kazuo
Tatum Jeremy B

Tchang-Brillet Lydia

Tozzi Gian Paolo

Tran Minh Nguyet

Trefftz Eleonore E

van Dishoeck Ewine F

van Regemorter Henri

van Rensbergen Walter

Varshalovich Dimitrij

Voelk Heinrich J

Volonte Sergio

Vujnovic Vladis

Weniger Schame

Wiese Wolfgang L

Wilson Robert

Winnewisser Gisbert

Wunner Guenter

Yoshino Kouichi

Young Louise Gray

$\mathrm{Yu}$ Yan

Zeippen Claude

Zeng Qin

Zirin Harold 


\title{
Composition of Commission 15
}

\author{
Physical Study of Comets \& Minor Planets \\ Etude physique des comètes $\&$ petites planètes
}

$\begin{array}{ll}\text { PRESIDENT: } & \text { Keller Horst Uwe } \\ \text { VICE-PRESIDENT: } & \text { Tedesco Edward F }\end{array}$

\section{ORGANIZING COMMITTEE:}

Capria Maria Teresa

Kiselev Nikolai N

McFadden Lucy Ann

Meech Karen

\section{MEMBERS:}

A'Hearn Michael F

Allegre Claude

Altwegg Kathrin

Andrienko Dmitry A

Arnold James R

Arpigny Claude

Axford W Ian

Babadzhanov Pulat B

Bailey Mark Edward

Barker Edwin S

Barriot Jean-Pierre

Barucci Maria A

Bell Jeffrey $F$

Belskaya Irina $\mathrm{N}$

Belton Michael J S

Binzel Richard $P$

Birch Peter

Blamont Jacques-Emile

Bockelee-Morvan Dominique

Boehnhardt Hermann

Boice Daniel Craig

Bonev Tanyu

Bouska Jiri

Bowell Edward L G

Brandt John C

Brecher Aviva

Brown Robert Hamilton

Brownlee Donald E

Brunk William E

Buie Marc W

Buratti Bonnie J

Burlaga Leonard $F$

Burns Joseph A
Michalowski Tadeusz

Muinonen Karri

Sykes Mark Vincent

Campins Humberto

Capaccioni Fabrizio

Carruthers George R

Carsenty Uri

Carusi Andrea

Cellino Alberto

Ceplecha Zdenek

Cerroni Priscilla

Chandrasekhar T

Chapman Clark R

Chapman Robert D

Chen Daohan

Chernykh N S

Clairemidi Jacques

Clayton Geoffrey C

Clayton Robert $\mathrm{N}$

Clube S V M

Cochran Anita L

Cochran William David

Colom Pierre

Combi Michael R

Consolmagno Guy Joseph

Cosmovici Batalli C

Cremonese Gabriele

Cristescu Cornelia G

Crovisier Jacques

Cruikshank Dale P

Cuypers Jan

Danks Anthony C

Davies John K

de Almeida Amaury A

de Pater Imke

de Sanctis Giovanni
Watanabe Jun-ichi

West Richard M

Zappala Vincenzo

De Sanctis M Cristina

Debehogne Henri Sc

Delsemme Armand $\mathrm{H}$

Dermott Stanley F

Deutschman William A

Di Martino Mario

Donn Bertram D

Dotto Elisabetta

Dryer Murray

Dzhapiashvili Victor P

Encrenaz Therese

Ershkovich Alexander

Eviatar Aharon

Feldman Paul Donald

Fernandez Julio A

Ferrin Ignacio

Festou Michel C

Fitzsimmons Alan

Forti Giuseppe

Foryta Dietmar William

Froeschle Christiane D

Fujiwara Akira

Fulchignoni Marcello

Gammelgaard Peter

Gehrels Tom

Geiss Johannes

Gerard Eric

Gibson James

Giovane Frank

Gradie Jonathan Carey

Grady Monica M

Green Simon F

Greenberg J Mayo 
Greenberg Richard

Gronkowski Piotr M

Grossman Lawrence

Gruen Eberhard

Gustafson Bo A S

Hajduk Anton

Halliday Ian

Hanner Martha S

Hapke Bruce W

Harris Alan William

Hartmann William K

Harwit Martin

Hasegawa Ichiro

Haser Leo N K

Haupt Hermann F

Helin Eleanor Francis

Hestroffer Daniel

Huebner Walter F

Hughes David W

Huntress Wesley T

Ibadinov Khursandkul

Ibadov Subhon

Ip Wing-huen

Irvine William M

Isobe Syuzo

Ivanova Violeta

Jackson William M

Jockers Klaus

Johnson Torrence $\mathrm{V}$

Kaasalainen Mikko K

Karttunen Hannu

Keay Colin S 1

Keil Klaus

Kidger Mark R

Klacka Jozef

Kliem Bernhard

Knacke Roger F

Knezevic Zoran

Koeberl Christian

Kohoutek Lubos

Konopleva Varvara P

Korsun Pavlo P

Kowal Charles Thomas

Kozasa Takashi

Kresakova Margita

Krishna Swamy K S

Kristensen Leif Kahl

Lagage Pierre-Olivier

Lagerkvist Claes-Ingvar

Lamy Philippe
Lancaster Brown Peter

Lane Arthur Lonne

Larson Harold P

Larson Stephen M

Lazzarin Monica

Lebofsky Larry Allen

Lee Thyphoon

Levasseur-Regourd A.-C.

Liller William

Lillie Charles F

Lindsey Charles Allan

Lipschutz Michael E

Lissauer Jack J

Lopes-Gautier Rosaly

Lumme Kari A

Lupishko Dmitrij F

Lutz Barry L

Luu Jane

Ma Yuehua

Magnusson Per

Malaise Daniel J

Maran Stephen P

Marcialis Robert

Marsden Brian G

Marzari Francesco

Matson Dennis L

Matsuura Oscar T

McCord Thomas B

McCrosky Richard E

McDonnell J A M

McKenna Lawlor Susan

Meisel David D

Mendis Devamitta Asoka

Merline William J

Milani Andrea

Milet Bernard L

Millis Robert L

Moehlmann Diedrich

Moore Elliott P

Moroz Vasilis I

Morrison David

Mukai Tadashi

Mumma Michael Jon

Nakamura Akiko M

Nakamura Tsuko

Napier William M

Neff John S

Neukum G

Newburn Jr Ray L

Niedner Malcolm B
Ninkov Zoran

Noll Keith Stephen

O'Dell Charles R

Paolicchi Paolo

Parisot Jean-Paul

Pellas Paul

Pendleton Yvonne Jean

Perez de Tejada H A

Piironen Jukka O

Pilcher Carl Bernard

Pillinger Colin

Pittich Eduard M

Prialnik-Kovetz Dina

Proisy Paul E

Reitsema Harold J

Remy Battiau Liliane G A

Revelle Douglas Orson

Rickman Hans

Roemer Elizabeth

Rousselot Philippe

Russell Kenneth S

Russel Sara S

Sagdeev Roald Z

Saito Takao

Samarasinha Nalin $\mathrm{H}$

Scaltriti Franco

Schleicher David G

Schloerb F Peter

Schmidt H U

Schmidt Maarten

Schober Hans J

Scholl Hans

Schubart Joachim

Schulz Rita M

Sekanina Zdenek

Sharma A Surjalal

Sharp Christopher

Shimizu Mikio

Shkodrov V G

Shor Viktor A

Shul-man L M

Sivaraman K R

Sizonenko Yuri V

Smith Bradford A

Snyder Lewis E

Solc Martin

Spinrad Hyron

Steel Duncan I

Stern S Alan

Surdej Jean M G 
Svoren Jan

Swade Daryl Allen

Szego Karoly

Tacconi-Garman Lowell E

Takeda Hidenori

Tanabe Hiroyoshi

Tancredi Gonzalo

Tao Jun

Tatum Jeremy B

Terentjeva Alexandra K

Tholen David J

Tomita Koichiro

Toth Imre

Tozzi Gian Paolo

Valdes Sada Pedro A
Van Flandern Tom

Veeder Glenn J

Veverka Joseph

Vilas Faith

Walker Alistair Robin

Wallis Max K

Wasson John T

Wdowiak Thomas $\mathbf{J}$

Weaver Harold F

Wehinger Peter A

Weidenschilling S J

Weissman Paul Robert

Wells Eddie Neil

Wetherill George W

Whipple Fred L
Wilkening Laurel L

Williams Iwan $P$

Wood John A

Woolfson Michael M

Woszczyk Andrzej

Wyckoff Susan

Yabushita Shin A

Yang Jongmann

Yavnel Alexander A

Yeomans Donald $\mathrm{K}$

Zarnecki John Charles

Zellner Benjamin $\mathrm{H}$

Zhu Jin 


\section{Composition of Commission 16}

Physical Study of Planets \& Satellites/Etude physique des planètes \& satellites

PRESIDENT: $\quad$ Cruikshank Dale P

VICE-PRESIDENT: Courtin Regis

ORGANIZING COMMITTEE:

Belton Michael J S

Blanco Carlo

Consolmagno Guy Joseph

Coradini Angioletta

\section{MEMBERS:}

Akabane Tokuhide

Akimov Leonid

Alexandrov Yuri V

Appleby John F

Arthur David W G

Atkinson David $\mathrm{H}$

Atreya Sushil K

Barrow Colin $\mathrm{H}$

Batson Raymond Milner

Battaner Eduardo

Baum William A

Bazilevsky Alexandr T

Beebe Reta Faye

Beer Reinhard

Bell III James F

Bender Peter L

Ben-Jaffel Lofti

Berge Glenn L

Bergstralh Jay T

Bertaux Jean-Loup

Bezard Bruno G

Bhatia R K

Billebaud Francoise

Binzel Richard P

Blamont Jacques-Emile

Bondarenko Ludmila N

Bosma Pieter B

Boss Alan P

Boyce Peter B

Brahic Andre

Brecher Aviva

Broadfoot A Lyle

Brown Robert Hamilton

Brunk William E de Bergh Catherine

Ksanfomaliti Leonid V

McGrath Melissa Ann

Noll Keith Stephen

Buie Marc W

Buratti Bonnie J

Burba George A

Burns Joseph A

Calame Odile

Caldwell John James

Cameron Winifred S

Camichel Henri

Campbell Donald B

Capria Maria Teresa

Carsmaru Maria M

Catalano Santo

Chamberlain Joseph W

Chapman Clark R

Chen Daohan

Chevrel Serge

Clairemidi Jacques

Cochran Anita L

Colombo G

Combi Michael R

Connes Janine

Counselman Charles C

Davies Merton E

de Pater Imke

Dermott Stanley F

Dickel John R

Dickey Jean O'Brien

Dlugach Zhanna M

Dollfus Audouin

Drake Frank D

Drossart Pierre

Durrance Samuel $\mathrm{T}$

Dzhapiashvili Victor P

El Baz Farouk
Owen Tobias C

Spencer John R

Tejfel Viktor G

Elliot James L

Elston Wolfgang E

Encrenaz Therese

Epishev Vitali $P$

Eshleman Von R

Esposito Larry W

Ferrari Cecile

Fielder Gilbert

Fink Uwe

Fox Kenneth

Fox W E

Fujiwara Akira

Gautier Daniel

Gehrels Tom

Geiss Johannes

Gerard Jean-Claude M C

Giclas Henry L

Gierasch Peter J

Gold Thomas

Goldreich Peter

Goldstein Richard M

Goody R M

Gorenstein Paul

Gor'kavyi Nikolai

Goudas Constantine L

Green Jack

Grossman Lawrence

Guerin Pierre

Guest John E

Gulkis Samuel

Gurshtein Alexander A

Hagfors Tor

Halliday Ian

Hammel Heidi B 
Hanninen Jyrki

Harris Alan William

Hide Raymond

Holberg Jay B

Horedt Georg Paul

Hovenier J W

Hubbard William B

Hunt G E

Hunten Donald M

Irvine William M

Iwasaki Kyosuke

Johnson Torrence V

Jurgens Raymond $F$

Kiladze R I

Kim Yongha

Kislyuk Vitalij S

Kowal Charles Thomas

Kumar Shiv S

Kurt Vitaliy G

Kuzmin Arkadii D

Lane Arthur Lonne

Lara Luisa $\mathrm{M}$

Larson Harold P

Larson Stephen $\mathrm{M}$

Leikin Grigerij A

Lewis $\mathbf{J} \mathbf{S}$

Lissauer Jack J

Lockwood G Wesley

Lopes-Gautier Rosaly

Lopez-Moreno Jose Juan

Lopez-Puertas Manuel

Lopez-Valverde M A

Lumme Kari A

Lutz Barry L

Mahra H S

Marcialis Robert

Marov Mikhail Ya

Matson Dennis L

Matsui Takafumi

Mayer Cornell $\mathrm{H}$

McCord Thomas B

McElroy M B

McKinnon William Beall

Meadows A Jack
Mickelson Michael E

Mihalov John D

Mikhail Joseph Sidky

Millis Robert L

Miyamoto Sigenori

Moehlmann Diedrich

Molina Antonio

Moore Patrick

Moreno Fernando

Moroz Vasilis I

Morozhenko A V

Morrison David

Mosser Benoit

Mulholland John Derral

Mumma Michael Jon

Murphy Robert E

Nakagawa Yoshitsugu

Ness Norman F

Neukum G

Ottelet I J

Pang Kevin

Paolicchi Paolo

Petit Jean-Marc

Petropoulos Basil Ch

Pettengill Gordon $\mathrm{H}$

Pillinger Colin

Pokorny Zdenek

Potter Andrew E

Predeanu Irina

Rao M N

Rodionova Janna $\mathrm{F}$

Rodrigo Rafael

Roques Francoise

Ruskol Eugenia L

Saissac Joseph

Sanchez-Lavega Agustin

Schleicher David G

Schloerb F Peter

Schneider Nicholas M

Shapiro Irwin I

Shevchenko Vladislav V

Shimizu Mikio

Shimizu Tsutomu Emer

Shkuratov Yurii
Sicardy Bruno

Sinton William M

Sjogren William L

Smith Bradford A

Soderblom Larry

Sonett Charles P

Sprague Ann Louise

Stern S Alan

Stoev Alexei

Stone Edward C

Strobel Darrell F

Strom Robert G

Synnott Stephen P

Tchouikova Nadejda A

Terrile Richard John

Tholen David J

Trafton Laurence $M$

Tran-Minh Francoise

Tyler Jr G Leonard

van Allen James A

Van Flandern Tom

Veiga Carlos Henrique

Veverka Joseph

Vidmachenko Anatoliy $\mathrm{P}$

Walker Alta Sharon

Walker Robert M A

Wallace Lloyd V

Wamsteker Willem

Wasserman Lawrence $\mathrm{H}$

Wasson John T

Weidenschilling S J

Weimer Theophile P F

Wells Eddie Neil

Wetherill George W

Whitaker Ewen A

Williams Iwan P

Williams James $G$

Wood John A

Woolfson Michael M

Woszczyk Andrzej

Yoder Charles F

Young Andrew $\mathrm{T}$

Young Louise Gray

Zharkov Vladimir N 
Composition of Commission 19

Rotation of the Earth/Rotation de la Terre

PRESIDENT: Capitaine Nicole

VICE-PRESIDENT: Dehant Veronique

ORGANIZING COMMITTEE:

Beutler Gerhard
Brosche Peter
Brzezinski Aleksander
Fukushima Toshio
Gambis Daniel
Gross Richard Sewart

MEMBERS:

Arabelos Dimitrios

Arias Elisa Felicitas

Bang Yong Gol

Banni Aldo

Barlier Francois E

Bender Peter $\mathrm{L}$

Bizouard Christian

Blinov Nikolai S

Boucher Claude

Bougeard Mireille L

Boytel Jorge Del Pino

Cazenave Anny

Chiumiento Giuseppe

De Biasi Maria S

Debarbat Suzanne V

Defraigne Pascale

Dejaiffe Rene J

Dick Wolfgang

Dickey Jean O'Brien

Dickman Steven R

Djurovic Dragutin M

El Shahawy Mohamad

Feissel Martine

Fliegel Henry F

Fong Chugang

Fujishita Mitsumi

Gao Buxi

Gaposchkin Edward M

Gayazov Iskander S

Gontier Anne-Marie

Gozhy Adam

Groten Erwin
Hefty Jan

Huang Cheng

Malkin Zinovy M

McCarthy Dennis D

Poma Angelo

Ray James R

Ron Cyril

Sidorenkov Nikolay S

Soffel Michael

Yatskiv Ya S

Morrison Leslie V

Mueller Ivan I

Naumov Vitalij A

Newhall X X

Niemi Aimo

Ooe Masatsugu

Paquet Paul Eg

Pejovic Nadezda R

Pesek Ivan

Petit Gerard

Picca Domenico

Pilkington John D H

Popelar Josef

Proverbio Edoardo

Randic Leo

Robertson Douglas S

Rochester Michael G

Roosbeek Fabian

Ruder Hanns

Rusu I

Rykhlova Lidija V

Sadzakov Sofija

Sanchez Manuel

Sasao Tetsuo

Sato Koichi

Schillak Stanislaw

Schutz Bob Ewald

Sekiguchi Naosuke

Sevarlic Branislav M

Sevilla Miguel J

Shapiro Irwin I

Shelus Peter J 
Smith Humphry M

Souchay Jean

Stanila George

Stavinschi Magdalena

Stephenson F Richard

Sugawa Chikara

Tapley Byron D

Tarady Vladimir K

Tsao Mo

Veillet Christian

Vicente Raimundo $\mathrm{O}$
Vondrak Jan

Wang Kemin

Wang Zhengming

Wilkins George A

Williams James $G$

Wilson P

Wu Shouxian

Wuensch Johann Jakob

Xiao Naiyuan

$\mathrm{Xu}$ Jiayan

$\mathrm{Xu}$ Tong-Qi
Yang Fumin

Ye Shuhua

Yokoyama Koichi

Yumi Shigeru

Zhang Guo-Dong

Zhang Zhongping

Zhao Ming

Zhong Min

Zhou Yonghong

Zhu Yaozhong

Zhu Yonghe 


\section{Composition of Commission 20}

Positions \& Motions of Minor Planets, Comets \& Satellites

Positions \& mouvements des petites planètes, des comètes $\&$ des satellites

PRESIDENT: $\quad$ Bowell Edward L G

VICE-PRESIDENT: Valsecchi Giovanni B

\section{ORGANIZING COMMITTEE:}

Aksnes Kaare

Arlot Jean-Eudes

Carusi Andrea

Fernandez Julio A

\section{MEMBERS:}

A'Hearn Michael F Abalakin Victor $\mathrm{K}$

Aikman G Chris 1

Babadzhanov Pulat B

Baggaley William J

Bailey Mark Edward

Batrakov Yuri V

Bec-Borsenberger Annick

Benest Daniel

Bien Reinhold

Blanco Carlo

Blow Graham L

Boerngen Freimut

Branham Richard L

Burns Joseph A

Calame Odile

Carpino Mario

Chapront-Touze Michelle

Chernykh N S

Chio Chol Zong

Chodas Paul Winchester

Cristescu Cornelia G

de Sanctis Giovanni

Debehogne Henri Sc

Delsemme Armand $\mathrm{H}$

Dollfus Audouin

Donnison John Richard

Dourneau Gerard

Doval Jorge Perez

Dunham David W

Dvorak Rudolf

Dybczynskif Piotr A

Edelman Colette
Lemaitre Anne

Marsden Brian G

Rickman Hans

Edmondson Frank K

Elliot James L

Elst Eric Walter

Emelianov Nikolaj V

Epishev Vitali P

Ferraz-Mello Sylvio

Ferreri Walter

Forti Giuseppe

Franklin Fred A

Fraser Brian D

Freitas Mourao R r

Froeschle Claude

Gehrels Tom

Gibson James

Giclas Henry L

Gilmore Alan C

Greenberg Richard

Hahn Gerhard J

Hainaut Olivier R

Harper David

Harris Alan William

Hasegawa Ichiro

Haupt Hermann F

He Miao-fu

Helin Eleanor Francis

Hemenway Paul D

Henrard Jacques

Hers Jan

Heudier Jean-Louis

Hurnik Hieronim

Hunukawa Kiitiro

Ianna Philip A

Isobe Syuzo
Shor Viktor A

Yeomans Donald K

Zhang Jiaxiang

Ivanova Violeta

Jacobson Robert A

Kazantsev Anatoly M

Khatisashvili Alfez Sh

Kiang Tao

Kilmartin Pamela

Kinoshita Hiroshi

Kisseleva Tamara P

Klemola Arnold R

Knezevic Zoran

Kohoutek Lubos

Kosai Hiroki

Kowal Charles Thomas

Kozai Yoshihide

Krasinsky George A

Kristensen Leif Kahl

Krolikowska-Soltan

Malgorzata

Kulikova Nelli V

Lagerkvist Claes-Ingvar

Lazzaro Daniela

Li Guangyu

Lieske Jay $\mathrm{H}$

Lindblad Bertil A

Lomb Nicholas Ralph

Lovas Miklos

Mahra H S

Manara Alessandro A

Matese John J

Maury Alain J

McCrosky Richard E

McMillan Robert S

McNaught Robert H 


Medvedev Yuriy D
Melita Mario D
Message Philip J
Milani Andrea
Milet Bernard L
Millis Robert L
Mintz Blanco Betty
Monet Alice K B
Moravec Zdenek
Morris Charles S
Muinonen Karri
Mulholland John Derral
Murray Carl D
Nacozy Paul E
Nakamura Tsuko
Nakano Syuichi
Neslusan Lubos
Nobili Anna M
Oterma Liisi
Overbeek Michiel Daniel
Owen Jr William Mann
Pandey A K
Pascu Dan
Pauwels Thierry
Pierce David Allen
Pittich Eduard M
Porubcan Vladimir
Pravec Petr
Protich Milorad B
Qiao Rongchuan
Qinchang Lin
Rajamohan R
Raju Vasundhara

Rapaport Michel
Reitsema Harold J
Roemer Elizabeth
Roeser Siegfried
Russell Kenneth S
Sato Isao
Sato Massae
Schmadel Lutz D
Schober Hans J
Scholl Hans
Schubart Joachim
Schuster William John
Seidelmann P Kenneth
Sekanina Zdenek
Shelus Peter J
Shen Kaixian
Shkodrov V G
Sinclair Andrew T
Sitarski Grzegorz
Sokolsky Andrej G
Solovaya Nina A
Soma Mitsuru
Standish E Myles
Steel Duncan I
Stellmacher Irene
Stokes Grant H
Sultanov G F
Svoren Jan
Synnott Stephen P
Tancredi Gonzalo
Tatum Jeremy B
Taylor Donald Boggia
Tholen David J

Thuillot William

Ticha Jana

Tomita Koichiro

Torkelsson Ulf J

Torres Carlos

Tsuchida Masayoshi

Van Flandern Tom

van Houten C J

van Houten-Groeneveld I

Vavrova Zdenka

Veillet Christian

Vieira Martins Roberto

Vienne Alain

Vu Duong Tuyen

Wasserman Lawrence $\mathrm{H}$

Weissman Paul Robert

West Richard M

Whipple Arthur L

Whipple Fred L

Wild Paul

Williams Gareth V

Williams Iwan P

Williams James $G$

Wroblewski Herbert

Yabushita Shin A

Yoshikawa Makoto

Yuasa Manabu

Zadunaisky Pedro E

Zagretdinov Renat

Zappala Vincenzo

Zhang Qiang

Zhu Jin

Ziolkowski Krzysztof 


\section{Composition of Commission 21}

\section{Light of the Night Sky/Lumière du ciel nocturne}

PRESIDENT: $\quad$ Lamy Philippe

VICE-PRESIDENT: Greenberg J Mayo

\section{ORGANIZING COMMITTEE:}

$\begin{array}{lll}\text { Bowyer C Stuart } & \text { Hanner Martha S } & \text { Matsumoto Toshio } \\ \text { Dwek Eli } & \text { Leinert Christoph } & \text { Mikhail Joseph Sidky } \\ \text { Gustafson Bo A S } & \text { Levasseur-Regourd A.-C. } & \text { Mukai Tadashi }\end{array}$

\section{MEMBERS:}

Abraham Peter

Angione Ronald J

Baggaley William J

Banos Cosmas J

Belkovich Oleg I

Blamont Jacques-Emile

Broadfoot A Lyle

Chamberlain Joseph W

Clairemidi Jacques

Dermott Stanley F

d'Hendecourt Louis

Dodonov Serguej

Dubin Maurice

Dufay Maurice

Dumont Rene

Dunkelman Lawrence

Eichhorn Guenther

Elsaesser Hans

Feldman Paul Donald

Fujiwara Akira

Gadsden Michael

Galperin Yuri I

Giovane Frank

Gruen Eberhard

Harwit Martin

Hauser Michael G

Hecht James $\mathrm{H}$

Henry Richard C

Hofmann Wilfried

Hong Seung Soo

Hurwitz Mark V

Ivanov Kholodny Goz S
Jackson Bernard V
James John F
Joubert Martine
Karygina Zoya V
Kopylov Alexander
Koutchmy Serge
Kulkarni Prabhakar V
Leger Alain
Lemke Dietrich
Lillie Charles F
Lopez-Gonzalez Maria J
Lopez-Moreno Jose Juan
Lopez-Puertas Manuel
Lumme Kari A
Maihara Toshinori
Mather John Cromwell
Mattila Kalevi
Maucherat J
McDonnell J A M
Misconi Nebil Yousif
Morgan David H
Muinonen Karri
Mukai Sonoyo
Nawar Samir
Neizvestny Sergei
Nishimura Tetsuo
Paresce Francesco
Perrin Jean-Marie
Pfleiderer Jorg
Radoski Henry R

Reach William

Renard Jean-Baptiste

Robley Robert

Rodrigo Rafael

Rozhkovskij Dimitrij A

Sanchez Francisco

Sanchez-Saavedra M Luisa

Saxena P P

Schlosser Wolfhard

Schwehm Gerhard

Shefov Nicolai N

Soberman Robert K

Sparrow James G

Staude Hans Jakob

Sykes Mark Vincent

Tanabe Hiroyoshi

Toller Gary N

Toroshlidze Teimuraz I

Tyson John Anthony

Vrtilek Jan M

Wallis Max K

Weinberg J L

Wesson Paul S

Wilson P

Witt Adolf N

Wolstencroft Ramon D

Woolfson Michael M

Yamamoto Tetsuo

Yamashita Kojun

Zerull Reiner $\mathrm{H}$ 


\section{Composition of Commission 22}

Meteors, Meteorites \& Interplanetary Dust

Météores, météorites \& poussière interplanétaire

PRESIDENT: $\quad$ Porubcan Vladimir

VICE-PRESIDENT: Mann Ingrid

ORGANIZING COMMITTEE:

Asher David J

Baggaley William J

Borovicka Jiri

Grady Monica M

MEMBERS:

Abbott William N

Alexandrov Alexander $\mathrm{N}$

Babadzhanov Pulat B

Belkovich Oleg I

Bhandari N

Brownlee Donald E

Carusi Andrea

Ceplecha Zdenek

Cevolani Giordano

Clifton Kenneth St

Clube S V M

Cooper Timothy

Djorgovski Stanislav

Dubin Maurice

Elford William Graham

Forti Giuseppe

Glass Billy Price

Goswami J N

Gruen Eberhard

Gustafson Bo A S

Hajduk Anton

Hajdukova Maria

Halliday Ian

Hanner Martha S

Harvey Gale A

Hasegawa Ichiro

Hawkins Gerald S

Helin Eleanor Francis

Hey James Stanley

Hodge Paul W

Hong Seung Soo

Hughes David W

Jennison Roger C
Hawkes Robert Lewis

Jenniskens Petrus

Matheus Marie

Spurny Pavel

Jones James

Jopek Tadeusz Jan

Kalenichenko Valentin

Kapisinsky Igor

Kashscheyev B L

Keay Colin S 1

Koeberl Christian

Kokhirova Gulchehra I

Kramer Kh N

Kresakova Margita

Kruchinenko Vitaliy G

Lemaire Joseph F

Levasseur-Regourd A.-C.

Lindblad Bertil A

Lovell Sir Bernard

Marvin Ursula B

Mason John William

McCrosky Richard E

McDonnell J A M

McIntosh Bruce A

Meisel David D

Miles Howard G

Misconi Nebil Yousif

Murray C Andrew

Murray Carl D

Nakamura Takuji

Nakazawa Kiyoshi

Napier William M

Newburn Jr Ray L

Nuth Joseph A III

Pecina Petr

Pillinger Colin

Plavec Zdenka
Steel Duncan I

Watanabe Jun-ichi

Williams Iwan P

Yano Hajime

Polnitzky Gerhard

Poole Graham

Quesada Vinicio

Rajchl Jaroslav

Revelle Douglas Orson

Rickman Hans

Ripken Hartmut W

Russell John A

Sekanina Zdenek

Shao Cheng-yuan

Simek Milos

Soberman Robert K

Svestka Jiri

Svoren Jan

Tatum Jeremy B

Taylor Andrew

Tedesco Edward F

Terentjeva Alexandra K

Tomita Koichiro

Verniani Franco

Voloschuk Yuri I

Wang dechang

Weinberg $\mathrm{J} \mathrm{L}$

Wetherill George W

Whipple Fred L

Wood John A

Woolfson Michael M

$\mathrm{Xu}$ Pinxin

Yavnel Alexander A

Yeomans Donald K

Zhu Jin

Zvolankova Judita 
Composition of Commission 25

Stellar Photometry \& Polarimetry/Photométrie \& polarimétrie stellaire

PRESIDENT: $\quad$ Sterken Christiaan Leo

VICE-PRESIDENT: Landolt Arlo U

ORGANIZING COMMITTEE:

Adelman Saul J

Bastien Pierre

Bessell Michael S

Chen Wen Ping

Fabrika Sergei

Gilliland Ronald Lynn

MEMBERS:

Ables Harold D

Albrecht Rudolf

Anandaram Mandayam N

Angel J Roger P

Angione Ronald J

Anthony-Twarog Barbara J

Arnaud Jean-Paul

Arsenijevic Jelisaveta

Ashok N M

Aspin Colin

Axon David

Baldinelli Luigi

Baliyan Kiran S

Balona Luis Antero

Barnes III Thomas G

Barrett Paul Everett

Beck Rainer

Behr Alfred

Berdyugin Andrei V

Blanco Victor $M$

Blecha Andre Boris G

Bookmyer Beverly B

Borgman Jan

Borra Ermanno F

Breger Michel

Brown Douglas Nason

Buser Roland

Carney Bruce William

Carter Brian

Castelaz Micheal W

Celis Leopoldo

Connolly Leo Paul
Glass Ian Stewart

Graham John A

Hensberge Herman

Martinez Peter

Milone Eugene F

Cousins A W J

Coyne George V

Cramer Noel

Crawford David L

Cuypers Jan

Dachs Joachim

Dahn Conard Curtis

Danford Stephen C

Deshpande M R

Dolan Joseph F

Dubout Renee

Ducati Jorge Ricardo

Ducourant Christine

Efimov Yuri

Fabregat Juan

Feinstein Alejandro

Fernie J Donald

Forte Juan Carlos

Gallouet Louis

Gehrz Robert Douglas

Genet Russel M

Gerbaldi Michele

Ghosh S K

Golay Marcel

Goy Gerald

Grauer Albert D

Grenon Michel

Grewing Michael

Guetter Harry Hendrik

Gutierrez-Moreno A

Hall Douglas S

Hauck Bernard
Sekiguchi Kazuhiro

Stetson Peter B

Straizys V

Walker William S G

Warren Jr Wayne $\mathbf{H}$

Hayes Donald S

Heck Andre

Hilditch Ronald W

Huang Lin

Hubrig Swetlana

Huovelin Juhani

Hyland A R Harry

Irwin Alan W

Iyengar K V K

Jerzykiewicz Mikolaj

Jordi Carme

Joshi Umesh C

Kawara Kimiaki

Kazlauskas Algirdas S

Kepler S O

Kilkenny David

King Ivan R

Knude Jens Kirkeskov

Koch Robert H

Kornilov Victor G

Kulkarni Prabhakar V

Kunkel William E

Kurtz Donald Wayne

Labhardt Lukas

Landstreet John D

Laskarides Paul G

Lazauskaite Romualda

Lemke Michael

Lenzen Rainer

Leroy Jean-Louis

Li Sin Hyong

Linde Peter 
Lockwood G Wesley

Lub Jan

Luna Homero G

Maitzen Hans M

Manfroid Jean

Markkanen Tapio

Marraco Hugo G

Masani A

Maslennikov Kirill L

Mathys Gautier

Mayer Pavel

McLean Ian S

Mendoza V Eugenio E

Menzies John W

Mianes Pierre

Miller Joseph S

Mintz Blanco Betty

Moffett Thomas J

Moreno Hugo

Morris Stephen C

Mourard Denis

Muller Andre B

Mumford George $S$

Nicolet Bernard

Noguchi Kunio

Notni $\mathbf{P}$

Oblak Edouard

Oestreicher Roland

Orsatti Ana M

Page Arthur

Pedreros Mario

Pel Jan Willem
Penny Alan John

Perry Charles L

Pfau Werner

Pfeiffer Raymond J

Philip A G Davis

Piirola Vilppu E

Platais Imants $\mathrm{K}$

Pokrzywka Bartlomiej

Rao P Vivekananda

Raveendran A V

Reglero-Velasco Victor

Richardson Lorna Logan

Robb Russell M

Robinson Edward Lewis

Romanyuk Yaroslav O

Roslund Curt

Rufener Fredy G

Sarma M B K

Schmidt Edward G

Schoeneich W

Schuster William John

Shakhovskoj Nikolay M

Shawl Stephen J

Smyth Michael J

Snowden Michael

Steinlin Uli

Stock Jurgen D

Stockman Jr Hervey S

Stone Remington P S

Strohmeier Wolfgang

Sudzius Jokubas

Sullivan Denis John
Szkody Paula

Szymanski Michal

Tandon S N

Tapia-Perez Santiago

Tinbergen Jaap

Todoran Ioan

Tokunaga Alan Takashi

Tolbert Charles $\mathbf{R}$

Ulrich Bruce T

Ureche Vasile

Vardanian R A

Vaughan Arthur $\mathrm{H}$

Verma R P

Visvanathan Natarajan

Vrba Frederick J

Walker Alistair Robin

Walraven $\mathrm{Th}$

Wang Chuanjin

Weiss Werner W

Weistrop Donna

Wesselius Paul R

White Nathaniel $M$

Wielebinski Richard

Willstrop Roderick V

Winiarski Maciej

Woo Jong Ok

Wramdemark Stig S

Yamashita Yasumasa

Yao Yongqiang

Yin Jisheng

Young Andrew T

Ziznovsky Jozef 
Composition of Commission 26

Double \& Multiple Stars/Etoiles doubles \& multiples

PRESIDENT: $\quad$ Scarfe Colin D

VICE-PRESIDENT: Hartkopf William I

ORGANIZING COMMITTEE:

Armstrong John Thomas Ling Josefina F.

Valtonen Mauri J

Fekel Francis C

Mathieu Robert D

Zinnecker Hans

Lampens Patricia

MEMBERS:

Abt Helmut A

Allen Christine

Anosova Joanna Argyle P E

Hakkila Jon Eric

Halbwachs Jean Louis

Herrera Miguel Angel

Bacchus Pierre

Hershey John L

Hidayat Bambang

Bagnuolo Jr William G

Balega Yuri Yu

Batten Alan H

Hill Graham

Beavers Willet I

Bernacca Pietio L

Bonneau Daniel

Hindsley Robert Bruce

Hummel Christian Aurel

Hummel Wolfgang

Brosche Peter

Cabrita Ezequiel

Campbell Alison

Cester Bruno

Chen Wen Ping

Chen Zhen

Clarke Catherine

Couteau Paul

Culver Roger Bruce

Dadaev Aleksandr N

Docobo Jose A Durantez

Ianna Philip A

Jassur Davoud MZ

Kiselyov Alexej A

Kroupa Pavel

Latham David W

Lattanzi Mario G

Leinert Christoph

Lippincott Sarah Lee

Loden Kerstin R

Loden Lars Olof

Malkov Oleg Yu

Martin Eduardo

Dommanget J

Douglass Geoffrey G

Dunham David W

Ferrer Osvaldo Eduardo

Fletcher J Murray

Franz Otto G

Fredrick Laurence W

Mason Brian D

McAlister Harold A

Meyer Claude

Mikkola Seppo

Mohan Chander

Morbey Christopher L

Morbidelli Roberto

Freitas Mourao R r

Gatewood George

Morel Pierre-Jacques

Oblak Edouard

Gaudenzi Silvia

Geyer Edward H

Oswalt Terry D

Pannunzio Renato

Ghez Andrea

Peterson Deane $M$

Popovic Georgije

Poveda Arcadio

Prieto Cristina

Rakos Karl D

Reipurth Bo

Russell Jane L

Sagar Ram

Salukvadze G N

Scardia Marco

Schmidtke Paul C

Simon Michal

Sinachopoulos D

Smak Joseph I

Soderhjelm Staffan

Sowell James Robert

Stein John William

Szabados Laszlo

Tarasov Anatolii E

Terquem Caroline E

Tokovinin Andrej A

Torres Guillermo

Trimble Virginia L

Tsay Wean-Shun

Upgren Arthur R

Valbousquet Armand

van Altena William $F$

van der Hucht Karel A

van Dessel Edwin Ludo

Vaz Luiz Paulo Ribeiro

Walker Richard L

Wang Jiaji

Weis Edward W

Yan Lin-shan 


\section{Composition of Commission 27}

Variable Stars/Etoiles variables

\section{PRESIDENT: $\quad$ Christensen-Dalsgaard J}

VICE-PRESIDENT: Aerts Conny

\section{ORGANIZING COMMITTEE:}

Cacciari Carla

Cottrell Peter Ledsam

Guinan Edward Francis

Harmanec Petr

\section{MEMBERS:}

Abada-Simon Meil

Aizenman Morris L

Albinson James

Albrow Michael

Alfaro Emilio Javier

Allan David W

Alpar Ali

Ando Hiroyasu

Andrievsky Sergei

Antipova Lyudmila I

Antonello Elio

Antov Alexandar

Arellano Ferro Armando

Arkhipova Vera $P$

Arsenijevic Jelisaveta

Asteriadis Georgios

Avgoloupis Stavros

Baade Dietrich

Baglin Annie

Baker Norman $\mathrm{H}$

Balona Luis Antero

Barnes III Thomas G

Bartolini Corrado

Barwig Heinz

Bastien Pierre

Bateson Frank M OBE

Bath Geoffrey $T$

Bauer Wendy Hagen

Beaulieu Jean-Philippe R

Bedogni Roberto

Belmonte Aviles J A

Belserene Emilia P

Belvedere Gaetano
Kurtz Donald Wayne

Matthews Jaymie

Moskalik Pawel

Sasselov Dimitar D
Somasundaram Seetha

Torres Carlos Alberto

Welch Douglas L

Whitelock Patricia Ann

\begin{tabular}{|c|c|}
\hline Benkoe Jozsef M & Cook Kem Holland \\
\hline Benson Priscilla J & Coulson Iain M \\
\hline Berdnikov Leonid N & Coutts-Clement Christine \\
\hline Bersier David & Cox Arthur $N$ \\
\hline Berthomieu Gabrielle & Cutispoto Giuseppe \\
\hline Bessell Michael S & Cuypers Jan \\
\hline Bianchini Antonio & Danford Stephen C \\
\hline Bochonko D Richard & de Groot Mart \\
\hline Bolton C Thomas & Delgado Antonio Jesus \\
\hline Bond Howard E & Demers Serge \\
\hline Bopp Bernard W & Deng Li-Cai \\
\hline Boulon Jacques $\mathbf{J}$ & Deupree Robert G \\
\hline Bowen George H & Dickens Robert J \\
\hline Boyarchuk Alexander A & Diethelm Roger \\
\hline Boyarchuk Margarita $E$ & Donahue Robert Andrew \\
\hline Bradley Paul A. & Downes Ronald A \\
\hline Breger Michel & Dunlop Storm \\
\hline Brown Douglas Nason & Dupuy David L \\
\hline Buchler J Robert & Dziembowski Wojciech \\
\hline Burki Gilbert & Efremov Yuri N \\
\hline Busko Ivo C & El Basuny Ahmed Alawy \\
\hline Butler C John & Eskioglu A Nihat \\
\hline Butler Dennis & Evans Aneurin \\
\hline Caldwell John A R & Evans Nancy Remage \\
\hline Cameron Andrew Collier & Evren Serdar \\
\hline Casares Valazquez Jorge & Eyer Laurent \\
\hline Catchpole Robin M & Fadeyev Yuri A \\
\hline Chavira Enrique $\mathrm{Sr}$ & Feast Michael W \\
\hline Cherepashchuk Analily M & Feibelman Walter A \\
\hline Christy Robert F & Ferland Gary Joseph \\
\hline Cohen Martin & Fernie J Donald \\
\hline Connolly Leo P & Fitch Walter S \\
\hline Contadakis Michael & Friedjung Michael \\
\hline
\end{tabular}


Frolov Mikhail S

Gahm Goesta F

Garrido Rafael

Gascoigne S C B

Gasparian Lazar

Genet Russel M

Gershberg R E

Geyer Edward H

Gieren Wolfgang P

Gies Douglas R

Gillet Denis

Glagolevskij Juri V

Godoli Giovanni

Gorbatsky Vitalij G

Gosset Eric

Gough Douglas O

Goupil Marie-Jose

Graham John A

Grasdalen Gary L

Grinin Vladimir $\mathbf{P}$

Groenewegen Martin

Gurm Hardev S

Gursky Herbert

Guzik Joyce Ann

Hackwell John A

Haefner Reinhold

Haisch Bernhard Michael

Halbwachs Jean Louis

Hall Douglas S

Hamdy M A M

Handler Gerald

Hansen Carl J

Hao Jinxin

Hawley Suzanne Louise

Heiser Arnold M

Henden Arne Anthon

Herbig George $\mathrm{H}$

Hers Jan

Hesser James E

Hill Henry Allen

Hoffleit E Dorrit

Hojaev Alisher S

Horner Scott D

Houk Nancy

Howell Steve Bruce

Huenemoerder David P

Hutchings John B

Iben Jr Icko

Iijima Takashi

Ishida Toshihito

Ismailov Nariman $Z$
Jablonski Francisco

Jarzebowski Tadeusz

Jeffery Christopher S

Jerzykiewicz Mikolaj

Jewell Philip R

Jiang Biwei

Jiang Shi-Yang

Jones Albert F

Jurcsik Johanna

Kadouri Talib Hadi

Kambe Eiji

Kanamitsu Osamu

Kanbur Shashi

Kanyo Sandor

Karitskaya Eugenia A

Karovska Margarita

Karp Alan Hersh

Kawaler Steven D

Kazarovets Elena V

Kepler S O

Khaliullin Khabibrachman F

Kilkenny David

Kim Chulhee

Kim Tu-Whan

Kiplinger Alan L

Kippenhahn Rudolf

Kjeldsen Hans

Kjurkchieva Diana

Koen Marthinus

Koevari Zsolt

Kollath Zoltan

Kraft Robert $P$

Krautter Joachim

Kreiner Jerzy Marek

Krisciunas Kevin

Krzeminski Wojciech

Krzesinski Jerzy H

Kubiak Marcin A

Kuhi Leonard V

Kunjaya Chatief

Kunkel William E

Lago Maria Teresa V T

Lampens Patricia

Landolt Arlo U

Laney Clifton D

Lanning Howard Hugh

Lanza Antonino Francesco

Laskarides Paul G

Lawson Warrick

Lazaro Carlos

Le Bertre Thibaut $\mathbf{R}$
Leite Scheid Paulo

Leung Kam Ching

Li Yan

Li Zhiping

Little-Marenin Irene $\mathbf{R}$

Lockwood G Wesley

Longmore Andrew J

Lopez De Coca M D P

Lub Jan

Madore Barry Francis

Maeder Andre

Maffei Paolo

Mahmoud Farouk M A B

Mahra H S

Makarenko Ekaterina N

Mannino Giuseppe

Mantegazza Luciano

Margrave Jr Thomas Ewing

Martinez Peter

Masani A

Mattei Janet Akyuz

Mauche Christopher W

Mavridis L N

McGraw John T

Mcnamara Delbert $\mathrm{H}$

Melikian Norair D

Mennessier Marie-Odile

Metz Klaus

Michel Eric

Milone Eugene F

Milone Luis A

Minikulov Nasridin K

Mkrtichian David E

Moffett Thomas J

Mohan Chander

Morrison Nancy Dunlap

Mukai Koji

Mumford George S

Murdin Paul G

Nather R Edward

Neff John S

Niarchos Panayiotis

Nikolov Andrej

Nicolov Nikolai S

Nugis Tiit

Odgers Graham J

O'Donoghue Darragh

Olah Katalin

Opolski Antoni

Oswalt Terry D

Papaloizou John C B 
Paparo Margit

Papousek Jiri

Parsamyan Elma S

Parthasarathy Mudumba

Paterno Lucio

Pavlovski Kresimir

Percy John R

Petersen J Otzen

Petrov Peter $\mathbf{P}$

Pettersen Bjoern Ragnvald

Piirola Vilppu E

Pijpers Frank Peter

Plachinda Sergei I

Pollard Karen

Pont Frederic

Pop Alexandru Viorel

Pop Vasile

Pringle James E

Provost Janine

Pskovskij Juri P

Pugach Alexander F

Rakos Karl D

Rao N Kameswara

Reinsch Klaus

Renson P F M

Robinson Edward Lewis

Rodono Marcello

Rodriguez Eloy

Romano Giuliano

Romanov Yuri S

Rosenbush Alexander E

Rountree Janet

Russev Ruscho

Sadik Aziz R

Saha Abhijit

Samus Nikolai N

Sandmann William Henry

Sanyal Ashit

Sareyan Jean-Pierre

Sarma M B K

Sato Naonobu
Schlegel Eric Matthew

Schmidt Edward G

Schwartz Philip R

Schwarzenberg-Czerny A

Schwope Axel

Scuflaire Richard

Seeds Michael August

Shahul Hameed Mohin

Shakhovskaya Nadejda I

Shara Michael

Sharma Dharma Pal

Sherwood William A

Shobbrook Robert R

Sinvhal Shambhu Dayal

Smak Joseph I

Smeyers Paul

Smit Jan A

Smith Myron A

Soliman Mohamed Ahmed

Srivastava Ram Kumar

Starrfield Sumner

Stellingwerf Robert F

Stepien Kazimierz

Sterken Christiaan Leo

Stobie Robert S

Strassmeier Klaus G

Strohmeier Wolfgang

Strom Karen M

Strom Stephen E

Szabados Laszlo

Szatmary Karoly

Szecsenyi-Nagy Gabor

Szeidl Bela

Szkody Paula

Takata Masao

Takeuti Mine

Tammann Gustav Andreas

Tamura Shin'ichi

Tempesti Piero

Terzan Agop

Tjin-a-Djie Herman R E
Tremko Jozef

Tsioumis Alexandros

Tsvetkov Milcho $\mathrm{K}$.

Tsvetkova Katya

Turner David G

Tutukov A V

Tylenda Romuald

Udovichenko Sergei N

Usher Peter D

Valtier Jean-Claude

van Genderen Arnoud M

van Hoolst Tim

Ventura Rita

Verheest Frank

Viotti Roberto

Vogt Nikolaus

Waelkens Christoffel

Walker Edward N

Walker Merle F

Walker William S G

Wallerstein George

Walraven $\mathrm{Th}$

Warner Brian

Watson Robert

Webbink Ronald F

Wehlau Amelia

Weis Kerstin

Weiss Werner W

Williamon Richard M

Willson Lee Anne

Wilson Lionel

Wing Robert $\mathbf{F}$

Wood Peter R

Xiong Darun

Yudin Boris F

Zijlstra Albert

Zola Stanislaw

Zsoldos Endre

Zuckerman Ben M 


\section{Composition of Commission 28}

\section{Galaxies/Galaxies}

PRESIDENT: Okamura Sadanori

VICE-PRESIDENT: Sadler Elaine Margaret

\section{ORGANIZING COMMITTEE:}

Balkowski-Mauger Chantal

Bertola Francesco

Binney James J

Bruzual Gustavo

\section{MEMBERS:}

Aalto Susanne E

Ables Harold D

Abrahaman Hamlet V

Adler David Scott

Afanas'ev Viktor L

Aguero Estela L

Aguilar Luis A Chiu

Ahmad Farooq

Alcaino Gonzalo

Aldaya Victor

Alladin Saleh Mohamed

Allen Ronald J

Alloin Danielle

Alonso Maria Victoria

Amram Philippe

Andernach Heinz

Andrillat Yvette

Ann Hong Bae

Anosova Joanna

Aparicio Antonio

Arkhipova Vera $P$

Artamonov Boris $\mathrm{P}$

Athanassoula Evangelia

Ayani Kazuya

Azzopardi Marc

Bahcall John N

Bailey Mark Edward

Bajaja Esteban

Baldwin Jack A

Ballabh Goswami Mohan

Banhatti Dilip Gopal

Barbon Roberto

Barcons Xavier

Barthel Peter
Burstein David

de Zeeuw Pieter T

Fairall Anthony $P$

Heckman Timothy $M$

Bassino Lilia P
Basu Baidyanath
Battaner Eduardo
Battinelli Paolo
Baum William A
Beaulieu Sylvie F
Beck Rainer
Begeman Kor G
Bender Ralf
Benedict George F

Berczik Peter

Bergeron Jacqueline A

Bergvall Nils Ake Sigvard

Berkhuijsen Elly M

Berman Vladimir

Bettoni Daniela

Bian Yulin

Biermann Peter L

Bijaoui Albert

Binette Luc

Binggeli Bruno

Biretta John Anthony

Birkinshaw Mark

Bland-Hawthorn Jonathan

Blitz Leo

Block David Lazar

Blumenthal George R

Boisson Catherine

Boksenberg Alec

Bomans Dominik J

Borchkhadze Tengiz M

Borne Kirk D

Bosma Albert

Bottinelli Lucette
Lilly Simon J

Wielebinski Richard

Zou Zhenlong

Bower Gary Allen

Braccesi Alessandro

Braine Jonathan

Braun Robert

Brecher Kenneth

Bressan Alessandro

Bridges Terry J

Briggs Franklin

Brinkmann Wolfgang

Brinks Elias

Brodie Jean $P$

Brosch Noah

Brouillet Nathalie

Burbidge Eleanor Margaret

Burbidge Geoffrey R

Burns Jr Jack O'Neal

Buta Ronald J

Butcher Harvey R

Byrd Gene G

Byun Yong-Ik

Calderon Jesus

Calzetti Daniela

Campusano Luis E

Cannon Russell D

Canzian Blaise

Cao Xinwu

Capaccioli Massimo

Carigi Leticia

Carrillo Moreno Rene

Carswell Robert F

Carter David

Casoli Fabienne

Cayatte Veronique

Cellone Sergio Aldo 
Cepa Jordi

Chakrabarti Sandip K

Chamaraux Pierre

Chang Ruixiag

Charmandaris Vassilis

Chatterjee Tapan K

Chatzichristou Eleni T

Chen Jiansheng

Chen Zhencheng

Chiappini Moraes Leite

Cristina

Chiba Masashi

Chincarini Guido L

Chou Chih-Kang

Chu Yaoquan

Chugai Nikolai N

Cinzano Pierantonio

Clavel Jean

Cohen Ross D

Combes Francoise

Comte Georges

Contopoulos George

Cook Kem Holland

Corbin Michael R

Corwin Jr Harold G

Couch Warrick

Courtes Georges

Courvoisier Thierry J-L

Cowsik Ramanath

Crane Philippe

Cunow Barbara

da Costa Luiz A.N.

Danks Anthony C

Davidge Timothy J

Davidsen Arthur Falnes

Davies Rodney D

Davis Jonathan Ivor

Davis Marc

de Boer Klaas Sjoerds

de Bruyn A Ger

de Carvalho Reinaldo

de Silva L N K

Dejonghe Herwig Bert

Dekel Avishai

Demers Serge

Deng Zugan

Dennefeld Michel

Dettmar Ralf-Juergen

Diaz Angeles Isabel

Dickey John M
Dietrich Matthias

d'Odorico Sandro

Doi Mamoru

Dokuchaev Vyacheslav I

Donas Jose

Donner Karl Johan

D'Onofrio Mauro

Donzelli Carlos J

Dopita Michael A

Dottori Horacio A

Doyon Rene

Dressel Linda L

Dressler Alan

Drinkwater Michael J

Driver Simon P

Dufour Reginald James

Dultzin-Hacyan D

Dumont Anne-Marie

Durret Florence

Duval Marie-France

Edelson Rick

Edmunds Michael Geoffrey

Efstathiou George

Einasto Jaan

Ekers Ronald D

Elmegreen Debra Meloy

Elvis Martin S

Elvius Aina $M$

English Jayanne

Espey Brian Russell

Evans Robert

Fabbiano Giuseppina

Faber Sandra M

Fabricant Daniel G

Falco-Acosta Emilio E

Fall S Michael

Fan Junhui

Feast Michael W

Feinstein Carlos

Feitzinger Johannes

Ferland Gary Joseph

Ferrini Federico

Field George B

Filippenko Alexei V

Firmani Claudio A

Flin Piotr

Florsch Alphonse

Foltz Craig B

Forbes Duncan Alan

Ford Holland C Res
Ford Jr W Kent

Fouque Pascal

Fraix-Burnet Didier

Francis Paul

Freedman Wendy L

Freeman Kenneth C

Fricke Klaus

Fried Josef Wilhelm

Fritze Klaus

Fritze-von Alvensleben Uta

Frogel Jay Albert

Fuchs Burkhard

Fujita Yutaka

Fukugita Masataka

Funato Yoko

Gallagher III John S.

Galletta Giuseppe

Gamaleldin Abdulla I

Gardner Jonathan P

Garilli Bianca

Gascoigne S C B

Geller Margaret Joan

Georgiev Tsvetan

Gerhard Ortwin

Ghigo Francis D

Ghosh P

Giacani Elsa Beatriz

Gigoyan Kamo

Giovanardi Carlo

Giovanelli Riccardo

Glass Ian Stewart

Godlowski Wlodzimierz

Gonzalez Delgado Rosa M

Gonzalez Serrano J I

Goodrich Robert W

Gorgas Garcia Javier

Goss W Miller

Gottesman Stephen T

Gouguenheim Lucienne

Graham John A

Grebel Eva K.

Gregg Michael David

Griffiths Richard E

Gu Qiusheng

Gunn James E

Gurzadian Grigor A

Guseva Natalia G

Gyulbudaghian Armen L

Hagen-Thorn Vladimir A

Hamabe Masaru 
Hambrayan Valeri V

Hammer Francois

Han Cheongho

Hanami Hitoshi

Handa Toshihiro

Hara Tetsuya

Hardy Eduardo

Harms Richard James

Harnett Julieine

Hasan Hashima

He Xiang-Tao

Heidt Jochen

Held Enrico V

Helou George

Henning Patricia A

Henry Richard B C

Hensler Gerhard

Hewitt Adelaide

Hewitt Anthony V

Hickson Paul

Hintzen Paul Michael N

Hjalmarson Ake G

Hjorth Jens

Ho Luis C

Hodge Paul W

Hopp Ulrich

Horellou Cathy

Hou Jinliang

Hough James

Hu Fuxing

Hua Chon Trung

Huang Keliang

Huchra John Peter

Huchtmeier Walter $\mathrm{K}$

Humphreys Roberta M

Hunstead Richard W

Hunter Christopher

Hunter James $\mathrm{H}$

Ichikawa Shin-ichi

Ichikawa Takashi

Illingworth Garth D

Impey Christopher D

Infante Leopoldo

Irwin Judith

Israel Frank P

Issa Issa Aly

Ivison Robert J

Iwamuro Fumihide

Iye Masanori

Izotov Yuri
Jaffe Walter Joseph

Jang Minwhan

Jerjen Helmut

Jog Chanda J

Joly Monique

Jones Paul

Jones Thomas Walter

Jorgensen Inger

Joshi Umesh C

Joy Marshall J

Jugaku Jun

Jungwiert Bruno

Junkes Norbert

Junkkarinen Vesa $T$

Junor William

Kalinkov Marin P

Kalloglian Arsen $T$

Kandalian Rafik A

Kaneko Noboru

Karachentsev Igor D

Karachentseva Valentina

Karoji Hiroshi

Katgert Peter

Kaufman Michele

Keel William C

Kellermann Kenneth I

Kennicutt Robert C

Khachikian Edward Ye

Khanna Ramon

Khare Pushpa

King Ivan R

Kinman Thomas D

Kirshner Robert Paul

Klein Ulrich

Knapen Johan Hendrik

Knapp Gillian R

Kochhar Rajesh K

Kodaira Keiichi

Kodama Tadayuki

Kogoshvili Natela G

Kollatschny Wolfram

Kontizas Evangelos

Kontorovich Victor

Koo David C-Y

Koratkar Anuradha P

Koribalski Baerbel Silvia

Kormendy John

Kotilainen Jari

Kraan-Korteweg Renee C

Krause Marita
Krishna Gopal

Kron Richard G

Kumai Yasuki

Kunchev Peter

Kunth Daniel

Lancon Ariane

Larsen Soeren S

Larson Richard B

Laurikainen Eija

Layzer David

Le Fevre Olivier

Leacock Robert Jay

Ledlow Michael James

Lehto Harry $\mathbf{J}$

Leibundgut Bruno

Lequeux James

Li Jing

Li Xiaoqing

Lin Chia C

Lindblad Per Olof

Lo Kwok-Yung

Lopez Ericson D

Lopez Rosario

Lopez-Cruz Omar

Lord Steven Donald

Loup Cecile

Low Frank J

Lu Limin

Lugger Phyllis M

Luminet Jean-Pierre

Lynden-Bell Donald

Lynds Beverly $T$

Lynds Roger C

Ma Jun

Macalpine Gordon M

Maccagni Dario

Macchetto Ferdinando

Mackie Glen

Madden Suzanne

Madore Barry Francis

Magris C Gladis

Mahtessian Abraham P

Maiolino Roberto

Malagnini Maria Lucia

Malhotra Sageeta

Mannucci Filippo

Marcelin Michel

Marconi Alessandro

Marquez Isabel

Marr Jonathon M 
Marston Anthony Philip

Martin Maria Cristina

Martin Rene Pierre

Martinet Louis

Marziani Paolo

Masegosa Gallego J

Mathewson Donald S

Mauersberger Rainer

Maurice Eric N

Mayya Divakara

McBreen Brian Philip

McGaugh Stacy Sutton

Mediavilla Evencio

Meier David L

Meikle William P S

Meisenheimer Klaus

Mendes de Oliveira Claudia

Menon T K

Meusinger Helmut

Miley George K

Miller Hugh R

Miller Joseph S

Miller Richard H

Mirabel Igor Felix

Mizuno Takao

Moles Mariano J

Molinari Emilio

Moody Joseph Ward

Mori Masao

Moss Christopher

Mould Jeremy R

Mueller Volker

Munoz-Tunon Casiana

Muratorio Gerard

Murray Stephen S

Mushotzky Richard

Muzzio Juan C

Nair Sunita

Nakai Naomasa

Namboodiri P M S

Narlikar Jayant $\mathrm{V}$

Navarro Julio Fernando

Nedialkov Petko L

Ninkovic Slobodan

Nishikawa Ken-Ichi

Nityananda Ram

Noguchi Masafumi

Noonan Thomas W

Norman Colin A

Nulsen Paul
O'Connell Robert West

O'Dea Christopher P

Oemler Jr Augustus

Ohta Kouji

Olofsson Kjell

Oosterloo Thomas

Osman Anas Mohamed

Osterbrock Donald E

Pacholczyk Andrzej G

Palmer Philip

Palumbo Giorgio G C

Papayannopoulos Th

Park Jang-Hyun

Parker Quentin

Pastoriza Miriani G

Paturel Georges

Peimbert Manuel

Pello Roser Descayre

Perea-Duarte Jaime D

Perez Fournon Ismael

Perry Judith J

Peters William L III

Peterson Charles John

Petit Jean-Marc

Petrosian Artashes R

Petrov Georgy Trendafilov

Petuchowski Samuel J

Pfenniger Daniel

Phillips Mark M

Pikichian Hovhannes

Pizzella Alessandro

Pogge Richard William

Poggianti Bianca $M$

Popescu Cristina Carmen

Poveda Arcadio

Prabhu Tushar $\mathbf{P}$

Press William $\mathrm{H}$

Prevot-Burnichon MarieLouise

Prieur Jean-Louis

Pritchet Christopher J

Pronik I I

Pronik V I

Proust Dominique

Puerari Ivanio

Pustilnik Simon A

Quinn Peter

Quintana Hernan

Rafanelli Piero

Rampazzo Roberto
Rand Richard J

Raychaudhury Somak

Reaves Gibson

Reichert Gail Anne

Rephaeli Yoel

Reshetnikov Vladimir P

Richer Harvey B

Richstone Douglas O

Richter Gotthard

Rix Hans-Walter

Roberts Morton S

Roberts Jr William W

Roeser Hermann-Josef

Roos Nicolaas

Rosa Michael Richard

Rosado Margarita

Rose James Anthony

Rots Arnold H

Rozas Maite

Rubin Vera C

Rudnicki Konrad

Ryder Stuart

Sackett Penny

Sadat Rachida

Sadun Alberto Carlo

Sahibov Firuz H

Sakai Shoko

Sakamoto Kazushi

Sala Ferran

Salvador-Sole Eduardo

Sanahuja Blas

Sancisi Renzo

Sanders David B

Sanders Robert

Sanroma Manuel

Sansom Anne E

Sapre Ashok Kumar

Sarazin Craig $\mathbf{L}$

Sargent Wallace L W

Sasaki Minoru

Sasaki Toshiyuki

Saslaw William C

Sastry Shankara K

Savage Ann

Sawa Takeyasu

Schechter Paul L

Schmidt Maarten

Schmitt Henrique R

Schmitz Marion

Schucking Engelbert L 
Schultz G V

Schulz Hartmut

Schwarz Ulrich J

Schweizer Francois

Scorza de Appl Cecilia

Scoville Nicholas Z

Searle Leonard

Sellwood Jerry A

Sergeev Sergey G

Seshadri Sridhar

Setti Giancarlo

Shapovalova Alla I

Sharples Ray

Shaver Peter A

Shaya Edward J

Sherwood William A

Shields Gregory A

Shields Joseph C

Shimasaku Kazuhiro

Shostak G Seth

Shukurov Anvar

Sigurdsson Steinn

Sil'chenko Olga K

Sillanpaa Aimo Kalevi

Simien Francois

Simkin Susan M

Singh Kulinder Pal

Skillman Evan D

Slezak Eric

Smecker-Hane Tammy A

Smith Eric Philip

Smith Harding E

Smith Malcolm G

Smith Jr Harding E

Soares Domingos S L

Sobouti Yousef

Sohn Young-Jong

Soltan Andrzej Maria

Song Guoxuan

Sparks William Brian

Spinrad Hyron

Srinivasan G

Statler Thomas S

Staveley-Smith Lister

Steiman-Cameron Thomas

Stepanian A A

Stepanian Jivan A

Stiavelli Massimo

Stirpe Giovanna M

Stone Remington P S
Storchi-Bergman Thaisa

Strom Richard G

Strom Robert G

Subrahmanyam P V

Sugai Hajime

Sulentic Jack W

Sullivan Woodruff T

Sundin Maria I

Tacconi Linda J

Tacconi-Garman Lowell E

Tagger Michel

Takata Tadafumi

Takato Naruhisa

Tammann Gustav Andreas

Tanaka Yutaka D

Taniguchi Yoshiaki

Telles Eduardo

Terlevich Roberto Juan

Terzian Yervant

Thakur Ratna Kumar

Theis Christian

Thomasson Magnus

Thonnard Norbert

Thuan Trinh Xuan

Tiersch Heinz

Tifft William G

Tissera Patricia B

Tomita Akihiko

Tong $\mathrm{Yi}$

Toomre Alar

Tovmassian Hrant M

Toyama Kiyotaka

Traat Peeter

Tremaine Scott Duncan

Trimble Virginia L

Trinchieri Ginevra

Tsuchiya Toshio

Tsvetkov Dmitrii

Tully Richard Brent

Turner Edwin L

Tyson John Anthony

Ulrich Marie-Helene D

Urbanik Marek

Valentijn Edwin A

Vallenari Antonella

Valotto Carlos A

Valtonen Mauri J

van Albada Tjeerd S.

van den Bergh Sidney

van der Hulst Jan M van der Kruit Pieter C

van der Laan Harry

van der Marel Roeland P

van Driel Willem

van Gorkom Jacqueline $\mathrm{H}$

van Moorsel Gustaaf

van Woerden Hugo

Vansevicius Vladas

Varma Ram Kumar

Vauglin Isabelle

Vavilova Irina B

Veilleux Sylvain

Verdes-Montenegro Lourdes

Vermeulen Rene Cornelis

Veron Marie-Paule

Veron Philippe

Vigroux Laurent

Visvanathan Natarajan

Voglis Nikos

Vrtilek Jan M

Wada Keiichi

Wagner Stefan

Wakamatsu Ken-ichi

Walterbos Rene A M

Wang Tinggui

Wang Yiping

Ward Martin John

Weedman Daniel W

Weiler Kurt W

Welch Gary A

Westerlund Bengt $E$

White Simon David Manion

Whitmore Bradley C

Wielen Roland

Wiita Paul Joseph

Williams Barbara A

Williams Robert E

Williams Theodore B

Wills Beverley $\mathrm{J}$

Wills Derek

Wilson Andrew S

Windhorst Rogier A

Winkler Hartmut

Wisotzki Lutz

Wlerick Gerard

Woosley Stanley E

Worrall Diana Mary

Woudt Patrick A

Wozniak Herve

Wrobel Joan Marie 
Wynn-Williams C G

Xanthopoulos Emily

Xia Xiaoyang

Yamada Yoshiyaki

Yamagata Tomohiko

Yoshida Michitoshi

Young Judith Sharn
Zamorano Jaime

Zasov Anatole $\mathrm{V}$

Zavatti Franco

Zeilinger Werner W

Zepf Stephen Edward

Zhang Xiaolei

Zhang Yang
Zhou Xu

Zhou Youyuan

Ziegler Bodo L

Ziegler Harald

Zinn Robert J 


\section{Composition of Commission 29}

Stellar Spectra/Spectres stellaires

PRESIDENT: $\quad$ Mathys Gautier

VICE-PRESIDENT: $\quad$ Sneden Christopher A

ORGANIZING COMMITTEE:

Barbuy Beatriz

Friel Eileen D

Gratton Raffaela G

Heber Ulrich

MEMBERS:

Abhyankar Krishna D

Abia Carlos A

Abt Helmut A

Adelman Saul J

Aikman G Chris

Ake III Thomas Bellis

Alecian Georges

Aller Lawrence Hugh

Ambruster Carol

Andrillat Yvette

Appenzeller Immo

Arkharov Arkadi A

Artru Marie-Christine

Atac Tamer

Baade Dietrich

Baliunas Sallie L

Ballereau Dominique

Baratta Giovanni Battista

Basri Gibor B

Batalha Celso Correa

Bauer Wendy Hagen

Beckman John E

Bellas-Velidis Ioannis

Berger Jacques $G$

Bessell Michael S

Bikmaev Ilfan

Boehm Torsten D

Boesgaard Ann M

Boggess Albert

Bohlender David

Bond Howard E

Bonsack Walter $\mathrm{K}$

Bopp Bernard W

Bouvier Jerome
Landstreet John D

Molaro Paolo

Nissen Poul E

Parthasarathy Mudumba

Boyarchuk Alexander A

Brandi Elisande Estela

Breysacher Jacques

Briot Danielle

Brown Douglas Nason

Brown Paul James Frank

Bruhweiler Jr Fred C

Bruning David $\mathrm{H}$

Bues Irmela D

Burkhart Claude

Butler Keith

Carney Bruce William

Carpenter Kenneth G

Carter Bradley Darren

Cassatella Angelo

Castelli Fiorella

Catala Claude

Catalano Santo

Catchpole Robin M

Cayrel Roger

Cayrel de Strobel Giusa

Chavez-Dagostino Miguel

Cidale Lydia Sonia

Climenhaga John $\mathrm{L}$

Coluzzi Regina

Conti Peter S

Corbally Christopher

Cornide Manuel

Cottrell Peter Ledsam

Cowley Anne P

Cowley Charles R

Crowther Paul

Cunha Katia

da Silva Licio
Piskunov Nikolai E

Primas Francesca

Spite Francois M

Damineli Neto Augusto

Dawanas Djoni N

de Araujo Francisco $X$

de Castro Elisa

de Groot Mart

de Laverny Patrick

Divan Lucienne

Doazan Vera

Dolidze Madona V

Dragunova Alina V

Drake Natalia

Duncan Douglas Kevin

Dworetsky Michael M

Eenens Philippe

Faraggiana Rosanna

Feast Michael W

Felenbok Paul

Fernandez-Figueroa $\mathrm{M} \mathrm{J}$

Fitzpatrick Edward L

Floquet Michele

Foing Bernard $\mathrm{H}$

Foy Renaud

Franchini Mariagrazia

Francois Patrick

Frandsen Soeren

Freire Ferrero Rubens G

Friedjung Michael

Fujita Yoshio

Fullerton Alexander W

Garcia Lopez Ramon J

Garmany Catherine D

Garrison Robert F

Gautier Daniel

Gehren Thomas 
Gerbaldi Michele

Gershberg R E

Gesicki Krzysztof

Ghosh Kajal Kumar

Giampapa Mark S

Gilra Daya P

Giovannelli Franco

Glagolevskij Juri V

Glushneva Irina $\mathrm{N}$

Goebel John $\mathrm{H}$

Gonzalez Guillermo

Gopka Vera F

Grady Carol Anne

Gray David F

Greenstein Jesse L

Griffin R Elizabeth

Griffin Roger F

Gustafsson Bengt

Guthrie Bruce N G

Hack Margherita

Hanuschik Reinhard

Harmer Charles F W

Harmer Dianne L

Hartmann Lee William

Hashimoto Osama

Hearnshaw John B

Heintze J R W

Henrichs Hubertus $F$

Herbig George $\mathrm{H}$

Heske Astrid

Hessman Frederic Victor

Hill Grant

Hinkle Kenneth $\mathrm{H}$

Hirai Masanori

Hirata Ryuko

Hoeflich Peter

Houk Nancy

Houziaux Leo

Hron Josef

Huang Changchung

Hubeny Ivan

Hubert-Delplace A.-M.

Hubrig Swetlana

Huenemoerder David P

Hunger Kurt

Hyland A R Harry

Izumiura Hideyuki

Jiang Shi-Yang

Johnson Hollis R

Jordan Carole
Jugaku Jun

Khokhlova Vers L

Kipper Tonu

Kitchin Christopher R

Klochkova Valentina

Kodaira Keiichi

Kogure Tomokazu

Kolka Indrek

Kordi Ayman S

Kotnik-Karuza Dubravka

Koubsky Pavel

Kovachev B J

Kovtyukh Valery $\mathrm{V}$

Kraft Robert P

Krempec-Krygier Janina

Kwok Sun

Lago Maria Teresa V T

Lagrange Anne-Marie

Laird John B

Lambert David L

Lamers Henny J G L M

Lamontagne Robert

Lanz Thierry

Le Contel Jean-Michel

Lebre Agnes

Leckrone David S

Leedjaerv Laurits

Lester John B

Leushin Valery V

Levato Orlando Hugo

Liebert James W

Little-Marenin Irene $\mathrm{R}$

Lopes Dalton De faria

Lubowich Donald A

Luck R Earle

Lundstrom Ingemar

Magain Pierre

Magazzu Antonio

Maillard Jean-Pierre

Maitzen Hans M

Malaroda Stella M

Marilli Ettore

Massey Philip L

McGregor Peter John

Mcnamara Delbert H

Megessier Claude

Mickaelian Areg Martin

Mikulasek Zdenek

Moffat Anthony F J

Moos Henry Warren
Morossi Carlo

Morrison Nancy Dunlap

Napiwotzki Ralf

Neckel Heinz

Nicholls Ralph W

Niedzielski Andrzej

Niemela Virpi S

Nishimura Shiro Emer

Norris John

Nugis Tiit

Okazaki Atsuo T

Orlov Mikhail

Owocki Stanley Peter

Pagel Bernard E J

Pallavicini Roberto

Parsons Sidney B

Pasinetti Laura E

Pavlenko Yakov V

Pedoussaut Andre

Peery Benjamin F

Perrin Marie-Noel

Peters Geraldine Joan

Peterson Ruth Carol

Pilachowski Catherine

Pintado Olga Ines

Plavec Mirek J

Plez Bertrand

Polcaro V F

Polidan Ronald S

Porto de Mello Gustavo F

Praderie Francoise

Prinja Raman

Querci Francois R

Querci Monique

Raassen Ion

Rao N Kameswara

Rautela B S

Rauw Gregor

Rebolo Rafael

Rego Fernandez M

Reimers Dieter

Ringuelet Adela E

Romanyuk Iosif I

Rose James Anthony

Rossi Corinne

Rossi Lucio

Rossi Silvia C F

Rutten Robert J

Ryan Sean Gerard

Sadakane Kozo 
Sahade Jorge

Sanchez Almeida Jorge Sanwal Basant Ballabh Sareyan Jean-Pierre Schild Rudolph E

Scholz Gerhard

Schroeder Klaus Peter

Seggewiss Wilhelm

Shetrone Matthew C

Shore Steven N

Simon Theodore

Singh Mahendra

Sinnerstad Ulf E

Smalley Barry

Smith Graeme H

Smith Myron A

Smith Verne V

Snow Theodore $P$

Soderblom David R

Sonneborn George

Sonti Sreedhar Rao

Spite Monique

Stalio Roberto

Stateva Ivanka K
Stawikowski Antoni

Stecher Theodore P

Steffen Matthias

Stefl Stanislav

Stencel Robert Edward

St-Louis Nicole

Suntzeff Nicholas B

Svolopoulos Sotirios

Swensson John W

Swings Jean-Pierre

Takada-Hidai Masahide

Talavera A

Tautvaisiene Grazina

Thevenin Frederic

Tomov Toma V

Torrejon Jose Miguel

Tuominen Ilkka V

Underhill Anne B

Utsumi Kazuhiko

Valenti Jeff A.

Valtier Jean-Claude

van der Hucht Karel A

van Winckel Hans

van't Veer-Menneret Claude
Vasu-Mallik Sushma

Vilhu Osmi

Viotti Roberto

Vladilo Giovanni

Vogt Nikolaus

Vogt Steven Scott

Vreux Jean Marie

Wahlgren Glenn Michael

Wallerstein George

Waterworth Michael

Wegner Gary Alan

Wehinger Peter A

Weiss Werner W

Weniger Schame

Williams Peredur M

Wing Robert $F$

Wolf Bernhard

Wolff Sidney C

Wood H J

Wyckoff Susan

Yamashita Yasumasa

Yoshioka Kazuo

Zorec Juan

Zverko Juraj 


\section{Composition of Commission 30}

\section{Radial Velocities/Vitesses radiales}

PRESIDENT: $\quad$ Tokovinin Andrej A

VICE-PRESIDENT: Nordstroem Birgitta

\section{ORGANIZING COMMITTEE:}

Dravins Dainis

Hearnshaw John B

Levato Orlando Hugo

MEMBERS:

Abt Helmut A

Al-Malki M B

Andersen Johannes

Balona Luis Antero

Barbier-Brossat Madeleine

Batten Alan $\mathrm{H}$

Beavers Willet I

Beers Timothy C

Bernstein Hans Heinrich

Beuzit Jean-Luc

Boulon Jacques $\mathbf{J}$

Breger Michel

Burki Gilbert

Burnage Robert

Carney Bruce William

Carquillat Jean-Michel

Cochran William David

Crampton David

da Costa Luiz A.N.

Davis Marc

Davis Robert $\mathbf{J}$

de Jonge J K

de Medeiros Jose Renan

Dubath Pierre

Duflot Marcelle

Edmondson Frank $\mathrm{K}$

Fairall Anthony $P$

Fehrenbach Charles

Fekel Francis C

Fletcher J Murray

Florsch Alphonse

Foltz Craig B

Forveille Thierry

Freeman Kenneth C

Garcia Beatriz Elena

Georgelin Yvon P

Gilmore Gerard Francis
Mazeh Tsevi

Morrell Nidia

Quintana Hernan

Giovanelli Riccardo

Gnedin Yurij N

Gouguenheim Lucienne

Gray David F

Griffin Roger F

Halbwachs Jean Louis

Heintze J R W

Hewett Paul

Hilditch Ronald W

Hill Graham

Hrivnak Bruce J

Huang Changchung

Hube Douglas $P$

Hubrig Swetlana

Huchra John Peter

Imbert Maurice

Irwin Alan W

Isaak George $\mathrm{R}$

Kadouri Talib Hadi

Karachentsev Igor D

Khalesseh Bahram

Kraft Robert P

Latham David W

Lewis Brian Murray

Lindgren Harri

Marschall Laurence A

Maurice Eric N

Mayor Michel

McClure Robert D

McMillan Robert S

Melnick Gary J

Mermilliod Jean-Claude

Meylan Georges

Missana Marco

Mkrtichian David E

Morbey Christopher L

Oetken L
Smith Myron A

Szabados Laszlo

Udry Stephane

Pedoussaut Andre

Pellegrini Paulo S S

Pepe Francesco A

Perrier-Bellet Christian

Perry Charles L

Peterson Ruth Carol

Philip A G Davis

Popov Victor S

Pourbaix Dimitri

Preston George W

Prevot Louis

Rastorguev Alexey S

Ratnatunga Kavan U

Romanov Yuri S

Royer Frederic

Rubenstein Eric

Rubin Vera C

Samus Nikolai N

Sanwal N B

Scarfe Colin D

Scholz Gerhard

Sivan Jean-Pierre

Solivella Gladys Rebecca

Stefanik Robert

Stickland David J

Stock Jurgen D

Suntzeff Nicholas B

Szecsenyi-Nagy Gabor

Tonry John

van Dessel Edwin Ludo

Verschueren Werner

Vinko Jozsef

Walker Gordon A H

Wegner Gary Alan

Willstrop Roderick V

Yang Stephenson L S

Yoss Kenneth $M$ 


\section{Composition of Commission 31}

Time/L'heure

\section{PRESIDENT: $\quad$ Petit Gerard}

VICE-PRESIDENT: Matsakis Demetrios N

\section{ORGANIZING COMMITTEE:}

Beutler Gerhard

Brumberg Victor A

Leschiutta S

\section{MEMBERS:}

Abele Maris K

Afanasjeva Praskovya $M$

Allan David W

Alley Carrol O

Aoki Shinko

Arias Elisa Felicitas

Backer Donald Ch

Bender Peter $\mathbf{L}$

Blinov Nikolai S

Breakiron Lee Allen

Bruyninx Carine

Carter William Eugene

Chamberlain Joseph M

Dehant Veronique

Dick Wolfgang

Dickey Jean O'Brien

Douglas R J

Fallon Frederick W

Feissel Martine

Fliegel Henry F

Fujimoto Masa-Katsu

Fukushima Toshio

Gaignebet Jean

Gambis Daniel

Granveaud Michel

Grudler Pierre

Guinot Bernard R

Hall R Glenn

Han Tianqi
Luck John M

Paquet Paul Eg

Proverbio Edoardo

Hellwig Helmut Wilhelm

Hemmleb Gerhard

Hers Jan

Hosokawa Mizuhiko

Iijima Shigetaka

Ilyasov Yuri P

Jin Wenjing

Kakuta Chuichi

Klepczynski William J

Kolaczek Barbara

Kovalevsky Jean

Kwok Sun

Liang Zhonghuan

Lieske Jay $\mathrm{H}$

Liu Jinming

Lu Ben ui

Luo Dingchang

Luo Shi-Fang

Ma Zhenguo

Mathur B S

McCarthy Dennis D

Meinig Manfred

Melbourne William G

Melchior Paul J

Miao Yongrui

Millar Thomas J

Morgan Peter

Mueller Ivan I

Naumov Vitalij A
Qi Guanrong

Thomas Claudine

Newhall X X

Noel Fernando

Pilkington John D H

Pineau des Forets Guillaume

Popelar Josef

Pushkin Sergey B

Ray James R

Robertson Douglas S

Schuler Walter

Smith Humphry M

Smylie Douglas E

Song Jin'an

Stanila George

Tsuchiya Atsushi

Veillet Christian

Vicente Raimundo $\mathrm{O}$

Webrova Ludmila

Wilkins George A

Winkler Gernot M R

Wu Guichen

Wu Shouxian

Xu Bang-Xin

Yang Ke-jun

Yatskiv Ya S

Ye Shuhua

Zhang Jintong

Zheng Ying

Zhuang Qixiang 


\section{Composition of Commission 33}

Structure \& Dynamics of the Galactic System

Structure \& dynamique du système galactique

\section{PRESIDENT: $\quad$ Spergel David N}

VICE-PRESIDENT: Whitelock Patricia Ann

\section{ORGANIZING COMMITTEE:}

\author{
Efremov Yuri I \\ Evans Wyn \\ Flynn Chris \\ Gerhard Ortwin \\ Grindlay Jonathan E \\ Palous Jan
}

\section{MEMBERS:}

Acosta-Pulido Jose A

Adamson Andrew

Afanas'ev Viktor L

Agekjan Tateos A

Aguilar Luis A Chiu

Aizu Ko

Altenhoff Wilhelm J

Ambastha A K

Andersen Johannes

Antonov Vadim A

Aoki Shinko

Ardeberg Arne L

Ardi Eliani

Asteriadis Georgios

Athanassoula Evangelia

Bahcall John N

Baier Frank

Balazs Lajos G

Balbus Steven A

Balcells Marc

Baldwin John E

Banhatti Dilip Gopal

Barbanis Basil

Barberis Bruno

Bartasiute Stanislava

Bash Frank N

Basu Baidyanath

Baud Boudewijn

Berkhuijsen Elly M

Bienayme Olivier

Binney James J

Blaauw Adriaan

Blanco Victor $M$

Blitz Leo
Bloemen Johannes B G M

Boulon Jacques $\mathbf{J}$

Brand Jan

Bronfman Leonardo

Burke Bernard F

Burton W Butler

Caldwell John A R

Cane Hilary Vivien

Carpintero Daniel Diego

Carrasco Luis

Caswell James L

Cesarsky Catherine J

Cesarsky Diego A

Chapman Jessica

Chen $\mathrm{Li}$

Chen Zhen

Christodoulou Dmitris

Churchwell Edward B

Cincotta Pablo Miguel

Ciurla Tadeusz

Clemens Dan P

Clube S V M

Cohen Richard S

Comins Neil Francis

Contopoulos George

Costa Edgardo

Courtes Georges

Crampton David

Crawford David L

Creze Michel

Cubarsi Rafael

Cudworth Kyle McCabe

Cuisinier Francois C

Cuperman Sami
Perryman Michael A C

Wyse Rosemary F

Yuan Chi

Dauphole Bertrand

Davies Rodney D

Dawson Peter

de Jong Teije

Dejonghe Herwig Bert

Dekel Avishai

Dickel Helene R

Dickel John R

Dickman Robert L

Dieter Conklin Nannielou $\mathrm{H}$

Djorgovski Stanislav

Downes Dennis

Drilling John S

Ducati Jorge Ricardo

Ducourant Christine

Dzigvashvili R M

Edmondson Frank $\mathrm{K}$

Efremov Yuri N

Egret Daniel

Einasto Jaan

Elmegreen Debra Meloy

Elsaesser Hans

Evangelidis E

Faber Sandra M

Feast Michael W

Fehrenbach Charles

Feitzinger Johannes

Fenkart Rolf P

Figueras Francesca

Freeman Kenneth C

Fridman Aleksey M

Fuchs Burkhard

Fujimoto Masa-Katsu

Fujiwara Takao 
Fukunaga Masataka

Fux Roger M

Galletto Dionigi

Garzon Francisco

Gemmo Alessandra

Genkin Igor L

Genzel Reinhard

Georgelin Yvon P

Georgelin Yvonne M

Gilmore Gerard Francis

Goldreich Peter

Gomez Ana E

Gordon Mark A

Gottesman Stephen T

Grayzeck Edwin J

Grenon Michel

Gupta Sunil K

Habe Asao

Habing Harm J

Hakkila Jon Eric

Hamajima Kiyotoshi

Hanami Hitoshi

Hartkopf William I

Hawkins Michael R S

Hayli Avram

Heiles Carl

Herbst William

Herman Jacobus

Hernandez-Pajares Manuel

Hobbs Robert W

Hori Genichiro

Hozumi Shunsuke

Hron Josef

Hughes Victor A

Hulsbosch A N M

Humphreys Roberta M

Hunter Christopher

Ikeuchi Satoru

Inagaki Shogo

Innanen Kimmo A

Isobe Syuzo

Israel Frank $P$

Iwaniszewska Cecylia

Iye Masanori

Jablonka Pascale

Jackson Peter Douglas

Jahreiss Hartmut

Jalali Mir Abbas

Jasniewicz Gerard

Jiang Dongrong
Joench-Soerensen Helge

Jog Chanda J

Johnson Hugh M

Jonas Justin Leonard

Jones Derek H P

Kalandadze N B

Kalnajs Agris J

Kang Yong Hee

Kasumov Fikret K O

Kato Shoji

Kharadze E K

King Ivan $\mathrm{R}$

Kinman Thomas D

Klare Gerhard

Knapp Gillian R

Kormendy John

Kulsrud Russell M

Kutuzov Sergei A

Lafon Jean-Pierre J

Larson Richard B

Latham David W

Lecar Myron

Lee Hyung Mok

Lee Sang Gak

Li Jing

Liebert James W

Lin Chia C

Lindblad Per Olof

Lockman Felix J

Loden Kerstin R

Loden Lars Olof

MacConnell Darrell J

Macrae Donald A

Manchester Richard N

Marochnik L S

Martinet Louis

Martos Marco A

Mathewson Donald S

Matteucci Francesca

Mavridis L N

Mayor Michel

McGregor Peter John

Meatheringham Stephen

Mennessier Marie-Odile

Mezger Peter G

Mikkola Seppo

Miller Richard $\mathrm{H}$

Mirabel Igor Felix

Miyamoto Masanori

Moffat Anthony F J
Monet David G

Monnet Guy J

Morris Mark Root

Muench Guido

Nahon Fernand

Namboodiri P M S

Neckel Th

Nelson Alistair $\mathrm{H}$

Ninkovic Slobodan

Nishida Minoru

Nishida Mitsugu

Nordstroem Birgitta

Norman Colin A

Nuritdinov Salakhutdin

Oblak Edouard

Odenkirchen Michael

Oh Kap-Soo

Oja Tarmo

Ojha Devendra K

Okuda Haruyuki

Olano Carlos Alberto

Ollongren A

Ostriker Jeremiah P

Palmer Patrick E

Pandey A K

Papayannopoulos Th

Patsis Panos

Pauls Thomas Albert

Peimbert Manuel

Perek Lubos

Perry Charles L

Pesch Peter

Philip A G Davis

Pier Jeffrey R

Polyachenko Valerij L

Polymilis Chronis

Price R Marcus

Priester Wolfgang

Rabolli Monica

Raharto Moedji

Ratnatunga Kavan U

Reid Neill

Reif Klaus

Rich Robert Michael

Riegel Kurt W

Roberts Morton S

Roberts Jr William W

Robin Annie C

Robinson Brian $\mathbf{J}$

Rohlfs Kristen 
Rong Jianxiang

Rubin Vera C

Ruelas-Mayorga R A

Ruiz Maria Teresa

Rybicki George B

Saar Enn

Sala Ferran

Sanchez-Saavedra M Luisa

Sandquist Aage

Santillan Alfredo J

Sanz I Subirana Jaume

Sargent Anneila I

Schechter Paul L

Schmidt Hans

Schmidt K H

Schmidt Maarten

Schmidt-Kaler Theodor

Seggewiss Wilhelm

Seimenis John

Sellwood Jerry A

Serabyn Eugene

Shane William W

Shimizu Tsutomu Emer

Shu Frank H

Simonson S Christian

Sobouti Yousef

Solomon Philip M

Song Guoxuan

Soubiran Caroline
Sparke Linda

Spiegel E

Stecker Floyd W

Steinlin Uli

Stephenson C Bruce

Stibbs Douglas W N

Strobel Andrzej

Sturch Conrad R

Surdin Vladimir G

Svolopoulos Sotirios

Sygnet Jean-Francois

Tammann Gustav Andreas

Terzides Charalambos

The Pik-Sin

Thielheim Klaus O

Thomas Claudine

Tinney Christopher

Tobin William

Tomisaka Kohji

Tong Yi

Toomre Alar

Toomre Juri

Torra Jordi

Tosa Makoto

Trefzger Charles F

Tsioumis Alexandros

Tsujimoto Takuji

Upgren Arthur R

Valtonen Mauri J van der Kruit Pieter C

van Woerden Hugo

Vandervoort Peter $\mathrm{O}$

Varela Perez Antonia M

Vega $E$ Irene

Venugopal V R

Vergne Maria Marcela

Verschuur Gerrit L

Vetesnik Miroslav

Voroshilov V I

Wachlin Felipe Carlos

Weaver Harold F

Weistrop Donna

Westerhout Gart

Westerlund Bengt E

White Raymond E

Whiteoak John B

Whittet Douglas C B

Wielebinski Richard

Wielen Roland

Woltjer Lodewijk

Woodward Paul R

Wouterloot Jan Gerard A

Wramdemark Stig S

Yamagata Tomohiko

Yoshii Yuzuru

Younis Saad M

Zachilas Loukas

Zhang Bin 


\section{Composition of Commission 34}

\section{Interstellar Matter/Matière interstellaire}

PRESIDENT: $\quad$ Reipurth Bo
VICE-PRESIDENT:
Dyson John E

\section{ORGANIZING COMMITTEE:}

Bachiller Rafael
Franco Jose
Henning Thomas
Hua Chon Trung

MEMBERS:

Aannestad Per Arne

Abgrall Herve

Acker Agnes

Adams Fred

Aiad A Zaki

Aitken David K

Akabane Kenji A

Alcolea Javier

Aller Lawrence Hugh

Al-Mostafa Zaki A

Altenhoff Wilhelm J

Anantharamaiah Kuduvalli R

Andersson Bengt Goeran

Andrillat Yvette

Andronov Ivan

Anglada Guillem

Arkhipova Vera P

Arny Thomas T

Arthur Jane

Avery Lorne W

Axford W Ian

Azcarate Ismael $\mathrm{N}$

Baars Jacob W M

Baart Edward E

Baldwin John E

Balser Dana S

Baluteau Jean-Paul

Bania Thomas Michael

Barlow Michael J

Barnes Aaron

Bash Frank N

Baudry Alain

Becklin Eric E

Beckman John E
Kwok Sun

Millar Thomas J

Pineau des Forets Guillaume

Raymond John Charles

Beckwith Steven V W

Bedogni Roberto

Benaydoun Jean-Jacques

Bergeron Jacqueline A

Bergman Per-Goeran

Berkhuijsen Elly M

Bernat Andrew Plous

Bertout Claude

Bhatt H C

Bianchi Luciana

Bieging John Harold

Bignell R Carl

Binette Luc

Birkle Kurt

Black John Harry

Blades John Chris

Blair Guy Norman

Blair William $P$

Bless Robert C

Blitz Leo

Bloemen Johannes B G M

Bobrowsky Matthew

Bochkarev Nikolai G

Bode Michael F

Bodenheimer Peter

Boeshaar Gregory Orth

Boggess Albert

Bohlin Ralph C

Boisse Patrick

Boland Wilfried

Bontemps Sylvain

Borgman Jan

Boulanger Francois

Boumis Panayotis
Sofue Yoshiaki

Stasinska Grazyna

Tenorio-Tagle Guillermo

Vilchez Jose M

Bouvier Jerome

Brand Jan

Brand Peter W J 1

Breitschwerdt Dieter

Brinkmann Wolfgang

Bromage Gordon E

Brouillet Nathalie

Brown Ronald D

Bruhweiler Jr Fred C

Bryce Myfanwy

Bujarrabal Valentin

Burgess Alan

Burke Bernard F

Burton Michael G

Burton W Butler

Bystrova Natalija V

Bzowski Maciej

Cabrit Sylvie

Canto Jorge

Caplan James

Cappa de Nicolau Cristina

Capriotti Eugene R

Capuzzo Dolcetta Roberto

Carruthers George $\mathrm{R}$

Caselli Paola

Castaneda Hector

Caswell James L

Cecchi-Pellini Cesare

Centurion Marin Miriam

Cernicharo Jose

Cerruti'sola Monica

Cersosimo Juan Carlos

Cesarsky Catherine J

Cesarsky Diego A 


Chandra Suresh
Chen Yang
Cheng Kwang Ping
Chevalier Roger A
Chini Rolf
Chopinet Marguerite
Chu You-Hua
Churchwell Edward B
Ciardullo Robin
Clark Frank Oliver
Clegg Robin E S
Code Arthur D
Cohen Marshall H
Colangeli Luigi
Collin-Souffrin Suzy
Colomb Fernando R
Combes Francoise
Corbelli Edvige
Corradi Wagner J B
Costa Roberto D D
Costero Rafael
Courtes Georges
Cowie Lennox Lauchlan
Cox Pierre
Coyne George V
Crane Philippe
Crawford Ian Andrew
Crovisier Jacques
Cruvellier Paul E
Cudaback David D
Cugnon Pierre
Czyzak Stanley J
Dahn Conard Curtis
Dalgarno Alexander
Danks Anthony C
Danly Laura
Davies Rodney D
Davis Jonathan Ivor
de Almeida Amaury A
de Boer Klaas Sjoerds
de Gouveia Dal Pino E M
de Jong Teije
de la Noe Jerome
De Marco Orsola
Decourchelle Anne C
Deguchi Shuji
Deharveng Lise
Deiss Bruno M
Dennefeld Michel
Dewdney Peter E F

\begin{tabular}{|c|c|}
\hline d'Hendecourt Louis & Felli Marcello \\
\hline Di Fazio Alberto & Felten James E \\
\hline Dickel Helene R & Ferlet Roger \\
\hline Dickel John R & Ferriere Katia M \\
\hline Dickey John M & Ferrini Federico \\
\hline Dinerstein Harriet L & Fesen Robert A \\
\hline Dinh Van $M$ & Fiebig Dirk \\
\hline Disney Michael J & Field George B \\
\hline Djamaluddin Thomas & Fierro Julieta \\
\hline d'Odorico Sandro & Fischer Jacqueline \\
\hline Dokuchaev Vyacheslav I & Flannery Brian Paul \\
\hline Dokuchaeva Ólga D & Fleck Robert Charles \\
\hline Dominik Carsten & Flower David R \\
\hline Donn Bertram D & Folini Doris \\
\hline Dopita Michael A & Ford Holland C Res \\
\hline Dorschner Johann & Forster James Richard \\
\hline Dottori Horacio A & Fridlund Malcolm \\
\hline Downes Dennis & Frisch Priscilla \\
\hline Draine Bruce $T$ & Fuente Asuncion \\
\hline Drapatz Siegfried W & Fukui Yasuo \\
\hline Dreher John W & Fuller Gary A \\
\hline Dubner Gloria & Furniss Ian \\
\hline Dubout Renee & Gardner Francis F \\
\hline Dufour Reginald James & Garnett Donald Roy \\
\hline Duley Walter W & Gaume Ralph A \\
\hline Dupree Andrea $\mathbf{K}$ & Gaustad John E \\
\hline Duvert Gilles & Gay Jean \\
\hline Dwarkadas Vikram V & Geballe Thomas $\mathbf{R}$ \\
\hline Dwek Eli & Gehrels Tom \\
\hline Egan Michael P & Genzel Reinhard \\
\hline Eisloeffel Jochen & Georgelin Yvon P \\
\hline Elitzur Moshe & Gerard Eric \\
\hline Elliott Kenneth $\mathrm{H}$ & Gerin Maryvonne \\
\hline Elmegreen Bruce Gordon & Gerola Humberto \\
\hline Elmegreen Debra Meloy & Gezari Daniel Ysa \\
\hline Elvius Aina $\mathrm{M}$ & Ghanbari Jamshid \\
\hline Emerson James P & Giacani Elsa Beatriz \\
\hline Encrenaz Pierre $J$ & Gilra Daya P \\
\hline Escalante Vladimir & Giovanelli Riccardo \\
\hline Esipov Valentin F & Godfrey Peter Douglas \\
\hline Esteban Lopez Cesara & Goebel John H \\
\hline Evans Aneurin & Goldes Guillermo Victor \\
\hline Evans Neal J & Goldreich Peter \\
\hline Falgarone Edith & Goldsmith Donald W \\
\hline Falk Jr Sydney W & Goldsworthy Frederick A \\
\hline Falle Samuel A & Golovatyj Volodymyr \\
\hline Faulkner Donald J & Gomez Gonzalez Jesus \\
\hline Federman Steven Robert & Gonzales-Alfonso Eduardo \\
\hline Feibelman Walter A & Goodman Alyssa Ann \\
\hline Feitzinger Johannes & Gordon Courtney $\mathrm{P}$ \\
\hline
\end{tabular}


Gordon Mark A

Gosachinskij Igor V

Goss W Miller

Graham David A

Grasdalen Gary L

Gredel Roland

Greenberg J Mayo

Gregorio-Hetem Jane

Greisen Eric

Grewing Michael

Guelin Michel

Guertler Joachin

Guesten Rolf

Gull Theodore R

Gurzadian Grigor A

Guseinov O H

Habing Harm J

Hackwell John A

Hanami Hitoshi

Hardebeck Ellen G

Harrington J Patrick

Harris Alan William

Harris-Law Stella

Harten Ronald $\mathrm{H}$

Hartl Herbert

Hartquist Thomas Wilbur

Harvey Paul Michael

Hatchell Jennifer

Hayashi Saeko S

Haynes Raymond F

Hecht James H

Heiles Carl

Hein Righini Giovanna

Helfer H Lawrence

Helou George

Henkel Christian

Henny William John

Herbstmeier Uwe

Heydari-Malayeri Mohammad

Hidayat Bambang

Higgs Lloyd A

Hildebrand Roger $\mathrm{H}$

Hippelein Hans $\mathrm{H}$

Hirano Naomi

Hiromoto Norihisa

Hjalmarson Ake G

Hobbs Lewis M

Hoeglund Bertil

Hollenbach David John

Hollis Jan Michael
Hong Seung Soo

Houziaux Leo

Hovhannessian Rafik

Huggins Patrick J

Hughes Victor A

Hulsbosch A N M

Hutchings John B

Hutsemekers Damien

Hyung Siek

Irvine William M

Isobe Syuzo

Israel Frank P

Issa Issa Aly

Itoh Hiroshi

Iyengar K V K

Jabir Niama Lafta

Jacoby George $\mathrm{H}$

Jacq Thierry

Jaffe Daniel T

Jenkins Louise $F$

Johnson Fred M

Johnson Hugh M

Johnston Kenneth J

Jones Frank Culver

Jourdain de Muizon M

Jura Michael

Just Andreas

Juvela Mika J

Kafatos Minas

Kaftan May A

Kaifu Norio

Kaler James B

Kamijo Fumio

Kazes Ilya

Keene Jocelyn Betty

Kegel Wilhelm $\mathrm{H}$

Kennicutt Robert C

Kharadze E K

Khromov Gavriil S

Kimura Hiroshi

Kimura Toshiya

King David Leonard

Kirkpatrick Ronald C

Kirshner Robert Paul

Knacke Roger F

Knapp Gillian R

Knude Jens Kirkeskov

Kohoutek Lubos

Koike Chiyoe

Kondo Yoji
Koo Bon Chul

Koornneef Jan F

Korpi Maarit J

Kostyakova Elena B

Kozasa Takashi

Krautter Joachim

Kravchuk Sergei

Kreysa Ernst

Krishna Swamy K S

Kuiper Thomas B H

Kumar C Krishna

Kundu Mukul R

Kunth Daniel

Kunze Ruediger

Kutner Marc Leslie

Kwitter Karen Beth

Kylafis Nikolaos D

Lada Charles Joseph

Lafon Jean-Pierre J

Langer William David

Latter William B

Laureijs Rene J

Laurent Claudine

Le Squeren Anne-Marie

Lee Terence J

Leger Alain

Lehtinen Kimmo K

Leisawitz David

Lepine Jacques R D

Lequeux James

Leto Giuseppe

Leung Chun Ming

Likkel Lauren Jones

Liller William

Lin Chia C

Linke Richard Alan

Lis Dariusz C

Liseau Rene

Liszt Harvey Steven

Lizano-Soberon Susana

Lo Kwok-Yung

Lockman Felix J

Lopez Jose Alberto

Loren Robert Bruce

Lortet Marie-Claire

Louise Raymond

Lovas Francis John

Low Frank J

Lozinskaya Tat-yana A

Lucas Robert 
Luo Shaoguang

Lynds Beverly T

Mac Low Mordecai-Mark

Maciel Walter J

MacLeod John M

Maihara Toshinori

Mallik D C V

Mampaso Antonio

Manchado Arturo

Manchester Richard N

Manfroid Jean

Marston Anthony Philip

Martin Peter G

Martin Robert N

Martin-Pintado Jesus

Masson Colin R

Mather John Cromwell

Mathews William G

Mathewson Donald S

Mathis John S

Matsuhara Hideo

Matsumoto Tomoaki

Matsumura Masafumi

Mattila Kalevi

Mauersberger Rainer

McCall Marshall Lester

McCray Richard

McGee Richard X

McGregor Peter John

McKee Christopher F

McNally Derek

Meaburn John

Mebold Ulrich

Mehringer David Michael

Meier Robert R

Mellema Garrelt

Melnick Gary J

Mendez Roberto H

Mennella Vito

Menon T K

Menzies John W

Meszaros Peter

Mezger Peter G

Miller Joseph S

Milne Douglas K

Minn Young Key

Minter Anthony $\mathrm{H}$

Mitchell George F

Miyama Syoken

Mizuno Shun
Mo Jinger

Monin Jean-Louis

Montmerle Thierry

Moreno Corral Marco A

Morgan David $\mathrm{H}$

Morimoto Masaki

Morris Mark Root

Morton Donald C

Mouschovias Telemachos $\mathrm{Ch}$

Muench Guido

Mufson Stuart Lee

Murthy Jayant

Myers Philip C

Nagata Tetsuya

Nakada Yoshikazu

Nakagawa Takao

Nakamoto Taishi

Nakamura Fumitaka

Nakano Makoto

Nakano Takenori

Neugebauer Gerry

Nguyen-Quang Rieu

Nishi Ryoichi

Nordh Lennart $\mathrm{H}$

Norman Colin A

Nulsen Paul

Nussbaumer Harry

Nuth Joseph A III

O'Dell Charles R

O'Dell Stephen L

Ohtani Hiroshi

Okuda Haruyuki

Olofsson Hans

Omont Alain

Onaka Takashi

Onello Joseph S

Opendak Michael

Osaki Toru

Osborne John L

Osterbrock Donald E

Ozernoy Leonid M

Pagani Laurent $P$

Pagano Isabella

Pagel Bernard E J

Palla Francesco

Palmer Patrick E

Palumbo Maria Elisabetta

Panagia Nino

Pankonin Vernon Lee

Parker Eugene N
Parthasarathy Mudumba

Pauls Thomas Albert

Pecker Jean-Claude

Peimbert Manuel

Pena Miriam

Pendleton Yvonne Jean

Penzias Arno A

Pequignot Daniel

Perault Michel

Perinotto Mario

Persi Paolo

Peters William L III

Petrosian Vahe

Petuchowski Samuel J

Phillips John Peter

Phillips Thomas Gould

Pollacco Don

Pongracic Helen

Poppel Wolfgang G 1

Porceddu Ignazio E P

Pottasch Stuart R

Pound Marc W

Pouquet Annick

Prasad Sheo S

Preite Martinez Andrea

Price R Marcus

Pronik I I

Prusti Timo

Pskovskij Juri P

Puget Jean-Loup

Qin Zhihai

Radhakrishnan V

Raimond Ernst

Ratag Mezak Arnold

Rawlings Jonathan

Rengarajan $\mathrm{T} \mathrm{N}$

Reynolds Ronald J

Reynoso Estela M

Rickard Lee J

Robbins R Robert

Roberge Wayne G

Roberts Jr William W

Robinson Brian J

Robinson Garry

Roche Patrick F

Rodriguez Luis F

Roelfsema Peter

Roeser Hans-peter

Roger Robert S

Rogers Alan E E 
Rohlfs Kristen

Rosa Michael Richard

Rosado Margarita

Rose William K

Rouan Daniel

Roxburgh Ian W

Rozhkovskij Dimitrij A

Rozyczka Michal

Rubin Robert Howard

Ryabov Michael I

Sabano Yutaka

Sabbadin Franco

Sahu Kailash C

Salama Farid

Salinari Piero

Salpeter Edwin E

Salter Christopher John

Sanchez-Saavedra M Luisa

Sancisi Renzo

Sandell Goran Hans 1

Sandqvist Aage

Sarazin Craig L

Sargent Anneila I

Sarma N V G

Sato Fumio

Sato Shuji

Savage Blair D

Savedoff Malcolm P

Scalo John Michael

Scappini Flavio

Scarrott Stanley M

Schatzman Evry

Scherb Frank

Scheuer Peter A G

Schmid-Burgk J

Schmidt-Kaler Theodor

Schultz G V

Schulz Rolf Andreas

Schwartz Philip R

Schwartz Richard D

Schwarz Ulrich J

Scott Eugene Howard

Scoville Nicholas Z

Seaton Michael J

Seki Munezo

Sellgren Kristen

Sembach Kenneth R

Shane William W

Shao Cheng-yuan

Shapiro Stuart L
Sharpless Stewart

Shaver Peter A

Shawl Stephen J

Shcheglov P V

Shematovich Valery I

Sherwood William A

Shields Gregory A

Shmeld Ivar

Shu Frank H

Shull John Michael

Shull Peter Otto

Shustov Boris M

Silberberg Rein

Silich Sergey

Silk Joseph I

Silvestro Giovanni

Simons Stuart

Sitko Michael L

Sivan Jean-Pierre

Skilling John

Skulskyj Mychajlo Y

Sloan Gregory Clayton

Smith Barham W

Smith Craig H

Smith Michael

Smith Peter L

Smith Robert G

Snell Ronald L

Snow Theodore $\mathrm{P}$

Sobolev Andrej M

Sofia Sabatino

Sofia Ulysses J

Solc Martin

Solomon Philip M

Somerville William B

Stahler Steven W

Stanga Ruggero

Stanghellini Letizia

Stapelfeldt Karl R

Stecher Theodore $\mathrm{P}$

Stecklum Bringfried

Stenholm Lars

Stenholm Bjoern

Stone James McLellan

Strom Richard G

Suh Kyung-Won

Sun Jin

Suzuki Tomoharu

Swade Daryl Allen

Sylvester Roger
Szczerba Ryszard

Tafalla Mario

Takahashi Junko

Takakubo Keiya

Takano Toshiaki

Tamura Motohide

Tamura Shin'ichi

Tanaka Masuo

Taylor Kenneth N R

Terzian Yervant

Testi Leonardo

Thaddeus Patrick

The Pik-Sin

Thompson A Richard

Thonnard Norbert

Thronson Jr Harley Andrew

Tokarev Yurij V

Torrelles Jose M

Torres-Peimbert Silvia

Tosi Monica

Toth Laszlo V

Townes Charles Hard

Trammell Susan R

Treffers Richard R

Turner Barry $\mathrm{E}$

Turner Kenneth C

Ulrich Marie-Helene D

van der Hulst Jan M

van der Laan Harry

van Dishoeck Ewine $F$

van Gorkom Jacqueline $\mathrm{H}$

van Woerden Hugo

Vanden Bout Paul A

Varshalovich Dimitrij

Velazquez Pablo F

Verschuur Gerrit L

Viala Yves

Viallefond Francois

Vidal Jean-Louis

Vidal-Madjar Alfred

Viegas Aldrovandi S M

Visvanathan Natarajan

Viti Serena

Vogel Manfred

Volk Kevin

Voshchinnikov Nicolai

Vrba Frederick J

Wakker Bastiaan Pieter

Walker Gordon A H

Walmsley C Malcolm 
Walton Nicholas A

Wang Hongchi

Wang Junjie

Wannier Peter Gregory

Watt Graeme David

Weaver Harold F

Weiler Kurt W

Weinberger Ronald

Weisheit Jon C

Wendker Heinrich J

Wesselius Paul R

Weymann Ray J

White Glenn J

White Richard L

Whitelock Patricia Ann

Whiteoak John B

Whittet Douglas C B

Whitworth Anthony Peter

Wickramasinghe $\mathrm{N} \mathrm{C}$

Wiebe Dmitri

Williams David A
Williams Robert E

Williams Robin

Willis Allan J

Willner Steven Paul

Wilson Christine

Wilson Robert W

Wilson Thomas L

Winnberg Anders

Winnewisser Gisbert

Witt Adolf $N$

Wolff Michael J

Wolfire Mark Guy

Wolstencroft Ramon D

Wolszczan Alexander

Woltjer Lodewijk

Wood Douglas O S

Woodward Paul R

Woolf Neville J

Wootten Henry Alwyn

Wouterloot Jan Gerard A

Wright Edward L
Wu Chi Chao

Wynn-Williams C G

Yabushita Shin A

Yamamoto Satoshi

Yamamura Issei

Yamashita Takuya

Yan Jun

Yang Ji

York Donald G

Yorke Harold W

Yoshida Shigeomi

Younis Saad M

Yui Yukari Y

Zavagno Annie

Zealey William J

Zeilik Michael Ii

Zeng Qin

Zhang Cheng-Yue

Zhou ZhenpPu

Zimmermann Helmut

Zuckerman Ben M 


\section{Composition of Commission 35}

\section{Stellar Constitution/Constitution des étoiles}

PRESIDENT: VandenBerg Don

VICE-PRESIDENT: Dziembowski Wojciech

\section{ORGANIZING COMMITTEE:}

Christensen-Dalsgaard J Meynet Georges

Guzik Joyce Ann

Langer Norbert

\section{MEMBERS:}

Adams Mark T

Aiad A Zaki

Aizenman Morris L

Anand S P S

Angelov Trajko

Antia $\mathrm{H} \mathrm{M}$

Appenzeller Immo

Arai Kenzo

Arimoto Nobuo

Arnett W David

Arnould Marcel L

Audouze Jean

Baglin Annie

Baker Norman $\mathrm{H}$

Baym Gordon Alan

Becker Stephen A

Belmonte Aviles J A

Benz Willy

Bertelli Gianpaolo

Berthomieu Gabrielle

Bisnovatyi-Kogan Gennadij S

Blaga Christina $\mathrm{O}$

Bludman Sidney A

Bocchia Romeo

Bodenheimer Peter

Boehm Karl-Heinz

Bondi Hermann

Bono Giuseppe

Boss Alan P

Brassard Pierre

Bravo Eduardo

Bressan Alessandro

Brownlee Robert R

Bruenn Stephen W

Buchler J Robert
Michaud Georges J

Renzini Alvio

Burbidge Geoffrey R

Busso Maurizio

Callebaut Dirk K

Caloi Vittoria

Cameron Alastair G W

Canal Ramon $\mathrm{M}$

Caputo Filippina

Carson T R

Castellani Vittorio

Castor John I

Chan Kwing Lam

Chan Roberto

Charbonnel Corinne

Chechetkin Valerij M

Chevalier Claude

Chiosi Cesare S

Chitre Shashikumar M

Chkhikvadze Iakob N

Christy Robert F

Cohen Jeffrey M

Connolly Leo Paul

Cowan John J

D'Antona Francesca

Das Mrinal Kanti

Davis Jr Cecil G

de Greve Jean-Pierre

de Jager Cornelis

de Loore Camiel

de Medeiros Jose Renan

Dearborn David Paul K

Deinzer W

Deliyannis Constantine P

Demarque Pierre

Denissenkov Pavel

Despain Keith Howard
Saio Hideyuki

Tutukov A V

Vauclair Gerard P

Deupree Robert G

Dluzhnevskaya Olga B

Dominguez Inma

Durisen Richard H

Edwards Alan Ch

Eggleton Peter P

Eminzade T A

Endal Andrew $S$

Ergma E V

Eriguchi Yoshiharu

Ezer-Eryurt Dilhan

Fadeyev Yuri A

Faulkner Donald J

Faulkner John

Flannery Brian Paul

Fontaine Gilles

Forbes J E

Forestini Manuel

Fossat Eric

Foukal Peter V

Fujimoto Masayuki

Gabriel Maurice R

Gallino Roberto

Garcia Domingo

Gautschy Alfred

Geroyannis Vassilis S

Giannone Pietro

Gimenez Alvaro

Giridhar Sunetra

Glatzmaier Gary A

Gong Shumo

Goriely Stephane

Gough Douglas O

Goupil Marie-Jose 
Graham Eric

Greggio Laura

Guenther David

Gurm Hardev S

Hachisu Izumi

Hammond Gordon L

Han Zhanwen

Hashimoto Masa-aki

Hayashi Chushiro

Henry Richard B C

Hernanz Margarita

Hollowell David Earl

Hoshi Reiun

Hoyle Fred

Huang Runqian

Huggins Patrick J

Humphreys Roberta M

Iben Jr Icko

Iliev Ilian

Imbroane Alexandru

Imshennik Vladimir S

Isaak George $\mathrm{R}$

Isern Jorge

Ishizuka Toshihisa

Itoh Naoki

James Richard A

Kaehler Helmuth

Kaminishi Keisuke

Kato Mariko

Khozov Gennadij V

Kiguchi Masayoshi

King David S

Kippenhahn Rudolf

Kiziloglu Nilguen

Knoelker Michael

Kochhar Rajesh K

Koester Detlev

Kosovichev Alexander

Kovetz Attay

Kozlowski Maciej

Kumar Shiv S

Kwok Sun

Labay Javier

Lamb Susan Ann

Lamb Jr Donald Quincy

Lapuente Pilar Ruiz

Larson Richard B

Laskarides Paul G

Lasota Jean-Pierre

Lattanzio John
Lebovitz Norman $\mathbf{R}$

Lebreton Yveline

Lee Thyphoon

Lepine Jacques R D

Li Zongwei

Liebert James W

Linnell Albert P

Littleton John E

Livio Mario

Luo Guoquan

Maeder Andre

Maheswaran Murugesapillai

Mallik D C V

Marx Gyorgy

Masani A

Massevich Alla G

Matteucci Francesca

Mazurek Thaddeus John

Mazzitelli Italo

Melik-Alaverdian Yu

Mendes Luiz T

Mestel Leon

Meyer-Hofmeister Eva

Mitalas Romas Assoc

Miyaji Shigeki

Moellenhoff Claus

Mohan Chander

Monaghan Joseph J

Monier Richard

Moore Daniel R

Morgan John Adrian

Moskalik Pawel

Moss David L

Mowlavi Nami

Mueller Ewald

Nadyozhin Dmittris K

Nakamura Takashi

Nakano Takenori

Nakazawa Kiyoshi

Narasimha Delampady

Narita Shinji

Nasiri Sadollah Gheidari

Newman Michael John

Nishida Minoru

Noels Arlette

Nomoto Ken'ichi

Odell Andrew P

Ohyama Noboru

Okamoto Isao

Osaki Yoji
Ostriker Jeremiah $\mathrm{P}$

Oswalt Terry D

Paczynski Bohdan

Pamyatnykh Alexsey A

Pande Girish Chandra

Papaloizou John C B

Pearce Gillian

Phillips Mark M

Pines David

Pinotsis Antonis D

Plavec Mirek J

Pongracic Helen

Porfir'ev Vladimir V

Poveda Arcadio

Prentice Andrew J R

Prialnik-Kovetz Dina

Proffitt Charles R

Provost Janine

Qu Qinyue

Raedler K H

Ramadurai Souriraja

Rauscher Thomas

Ray Alak

Rayet Marc

Reeves Hubert

Ritter Hans

Rood Robert T

Rouse Carl A

Roxburgh Ian W

Ruben Gerhard

Sackmann Ingrid Juliana

Sakashita Shiro

Salpeter Edwin E

Santos Filipe D

Sato Katsuhiko

Savedoff Malcolm P

Savonije Gerrit Jan

Scalo John Michael

Schatten Kenneth H

Schatzman Evry

Schild Hansruedi

Schoenberner Detlef

Schutz Bernard F

Scuflaire Richard

Sears Richard Langley

Seidov Zakir F

Sengbusch Kurt V

Shaviv Giora

Shibahashi Hiromoto

Shibata Yukio 
Shustov Boris M

Sienkiewicz Ryszard

Signore Monique

Silvestro Giovanni

Sion Edward Michael

Smeyers Paul

Smith Robert Connon

Sobouti Yousef

Sofia Sabatino

Souffrin Pierre B

Sparks Warren M

Spiegel E

Sreenivasan S Ranga

Starrfield Sumner

Stellingwerf Robert F

Stibbs Douglas W N

Strittmatter Peter A

Suda Kazuo

Sugimoto Daiichiro

Sweet Peter A

Sweigart Allen V

Taam Ronald Everett

Takahara Mariko
Tassoul Monique

Thielemann Friedrich-Karl

Thomas Hans Christoph

Tjin-a-Djie Herman R E

Tohline Joel Edward

Toomre Juri

Tornambe Amedeo

Trimble Virginia $\mathrm{L}$

Truran Jr James W

Tscharnuter Werner M

Tuominen Ilkka V

Turck-Chieze Sylvaine

Uchida Juichi

Ulrich Roger K

Unno Wasaburo

Uus Undo

van den Heuvel Edward P J

van der Borght Rene

van der Raay Herman B

van Horn Hugh $M$

van Riper Kenneth A

Vardya M S

Vila Samuel C
Vilhu Osmi

Vilkoviskij Emmanuil Y

Ward Richard A

Weaver Thomas A

Webbink Ronald F

Weiss Achim

Weiss Nigel $\mathrm{O}$

Wheeler J Craig

Willson Lee Anne

Wilson Robert $\mathrm{E}$

Winkler Karl-Heinz A

Wood Matthew Alan

Wood Peter R

Woosley Stanley E

Xiong Darun

Yamaoka Hitoshi

Yorke Harold W

Yoshida Shin'ichirou

Yungelson Lev R

Zahn Jean-Paul

Zhevakin S A

Ziolkowski Janusz 


\section{Composition of Commission 36}

\section{Theory of Stellar Atmospheres/Théorie des atmosphères stellaires}

PRESIDENT: Dravins Dainis

VICE-PRESIDENT: Spite Monique

\section{ORGANIZING COMMITTEE:}

Ayres Thomas R

Barbuy Beatriz

Cram Lawrence Edward

Kiselman Dan

Landstreet John D

Pallavicini Roberto

\section{MEMBERS:}

Abbott David C

Abhyankar Krishna D

Aller Lawrence Hugh

Altrock Richard C

Arpigny Claude

Asplund Martin

Atanackovic-Vukmanovic

Olga Athay R Grant

Auer Lawrence $\mathrm{H}$

Auman Jason R

Avrett Eugene $\mathrm{H}$

Baade Dietrich

Baird Scott R

Balachandran Suchitra C

Baliunas Sallie L

Balona Luis Antero

Baschek Bodo

Basri Gibor B

Becker Sylvia

Bell Roger A

Berdyugina Svetlana V

Bernat Andrew Plous

Bertout Claude

Blanco Carlo

Bless Robert C

Blomme Ronny

Bodo Gianluigi

Boehm Karl-Heinz

Boehm-Vitense Erika

Boesgaard Ann M

Bopp Bernard W

Bowen George $\mathrm{H}$

Brown Alexander

Brown Douglas Nason

Bues Irmela D

Cameron Andrew Collier
Carbon Duane F
Carlsson Mats
Carson T R
Cassinelli Joseph P
Castelli Fiorella
Castor John I
Catala Claude
Catalano Francesco A
Catalano Santo
Cayrel Roger
Cayrel de Strobel Giusa
Chan Kwing Lam
Chen Peisheng
Chugai Nikolai N
Cidale Lydia Sonia
Conti Peter S
Cowley Charles R
Crivellari Lucio
Cruzado Alicia
Cugier Henryk
Cuntz Manfred
Cuny Yvette J
Davis Jr Cecil G
de Koter Alex
Deliyannis Constantine P
Dimitrijevic Milan
Doazan Vera
Domke Helmut
Donati Jean-Francois
Doyle John Gerard
Drake Stephen A
Duari Debiprosad
Dufton Philip L
Dupree Andrea K

Saio Hideyuki

Sneden Christopher A

Werner Klaus

Edvardsson Bengt

Elste Gunther H

Eriksson Kjell

Evangelidis E

Faraggiana Rosanna

Faurobert-Scholl Marianne

Feigelson Eric D

Fitzpatrick Edward L

Fontaine Gilles

Fontenla Juan Manuel

Foy Renaud

Freire Ferrero Rubens G

Friend David B

Frisch Helene

Frisch Uriel

Froeschle Christiane D

Gail Hans-Peter

Gallino Roberto

Garcia Lopez Ramon J

Gebbie Katharine B

Gesicki Krzysztof

Giampapa Mark S

Gigas Detlef

Glebocki Robert

Gokdogan Nuzhet

Gonzalez Jean-Francois

Gordon Charlotte

Gough Douglas O

Grant Ian P

Gratton Raffaela $G$

Gray David F

Greenstein Jesse L

Grevesse Nicolas

Grinin Vladimir P

Guedel Manuel 
Gussmann Ernst August

Gustafsson Bengt

Hack Margherita

Haisch Bernhard Michael

Hall Douglas S

Hamann Wolf-Rainer

Harper Graham M

Hartmann Lee William

Harutyunian Haik A

Hauschildt Peter H

Hearn Anthony G

Heasley James Norton

Heber Ulrich

Hekela Jan

Hoare Melvin

Hoeffner Susanne

Hoeflich Peter

Holweger Hartmut

Holzer Thomas Edward

Hotinli Metin

House Lewis L

Huang $\mathrm{He}$

Hubeny Ivan

Hunger Kurt

Hutchings John B

Ito Yutaka

Ivanov Vsevolod V

Jahn Krzysztof

Jatenco-Pereira Vera

Jevremovic Darko

Johnson Hollis R

Jordan Stefan

Judge Philip

Kadouri Talib Hadi

Kalkofen Wolfgang

Kamp Lucas Willem

Kandel Robert S

Karp Alan Hersh

Khokhlova Vers L

Klein Richard I

Kodaira Keiichi

Koester Detlev

Kolesov Alexander K

Kondo Yoji

Kontizas Evangelos

Krikorian Ralph

Krishna Swamy K S

Kubat Jiri

Kudritzki Rolf-Peter

Kuhi Leonard V
Kumar Shiv S

Kurucz Robert L

Lambert David L

Lamers Henny J G L M

Leibacher John

Liebert James W

Linnell Albert P

Linsky Jeffrey L

Liu Caipin

Loskutov Victor

Luck R Earle

Luttermoser Donald

Lyubimkov Leonid S

Madej Jerzy

Magazzu Antonio

Magnan Christian

Marlborough J M

Mashonkina Lyudmila

Massaglia Silvano

Mathys Gautier

Matsumoto Masamichi

Mauas Pablo

Michaud Georges J

Mihalas Dimitri

Miyamoto Sigenori

Mnatsakanian Mamikon A

Molaro Paolo

Muench Guido

Mukai Sonoyo

Musielak Zdzislaw E

Mutschlecner J Paul

Nagendra K N

Nagirner Dmitrij I

Narasimha Delampady

Nariai Kyoji

Neff John S

Nikoghossian Arthur G

Nordlund Aake

O'Mara Bernard J

Owocki Stanley Peter

Oxenius Joachim

Pacharin-Tanakun $P$

Pagel Bernard E J

Panek Robert J

Pasinetti Laura E

Pavlenko Yakov V

Pecker Jean-Claude

Peraiah Annamaneni

Perinotto Mario

Peters Geraldine Joan
Phillips John G

Pinsonneault Marc Howard

Pinto Philip Alfred

Piskunov Nikolai E

Pogodin Mikhail A

Pottasch Stuart R

Praderie Francoise

Puls Joahim

Querci Francois R

Querci Monique

Rachkovsky D N

Ramsey Lawrence W

Randich Sofia

Rangarajan K E

Rao D Mohan

Rauch Thomas

Reimers Dieter

Rodono Marcello

Ross John E R

Rostas Francois

Rovira Marta Graciela

Rucinski Slavek M

Rutten Robert J

Ryabchikova Tanya

Rybicki George B

Saito Kuniji

Sakhibullin Nail A

Sapar Arved

Sasselov Dimitar D

Sauty Christophe

Savanov Igor S

Schaerer Daniel

Scharmer Goeran Bjarne

Schmalberger Donald C

Schmid-Burgk J

Schmutz Werner

Schoenberner Detlef

Scholz M

Schrijver C J

Seaton Michael J

Sedlmayer Erwin

Shine Richard A

Shipman Harry L

Sigut Aaron T A

Simon Klaus Peter

Simon Theodore

Simonneau Eduardo

Skumanich Andre

Snezhko Leonid I

Snijders Mattheus A J 
Soderblom David R

Souffrin Pierre B

Spiegel E

Spite Francois $M$

Spruit Henk C

Stalio Roberto

Stauffer John Richard

Stee Philippe

Steffen Matthias

Stein Robert F

Stepien Kazimierz

Stern Robert Allan

Stibbs Douglas W N

Strom Stephen E

Szecsenyi-Nagy Gabor

Takeda Yoichi

Tautvaisiene Grazina

Thejll Peter Andreas

Toomre Juri
Traving Gerhard

Tsuji Takashi

Tuominen Ilkka V

Ueno Sueo

Uesugi Akira

Ulmschneider Peter

Underhill Anne B

Unno Wasaburo

Vakili Farrokh

van Regemorter Henri

van't Veer-Menneret Claude

van't-Veer Frans

Vardavas Ilias Mihail

Vardya M S

Vasu-Mallik Sushma

Vaughan Arthur $\mathrm{H}$

Velusamy $T$

Viik Tonu

Vilhu Osmi
Walter Frederick M

Watanabe Tetsuya

Waters Laurens B F M

Weber Stephen Vance

Wehrse Rainer

Weidemann Volker

Wellmann Peter

White Richard L

Wickramasinghe $\mathrm{N} \mathrm{C}$

Willson Lee Anne

Wilson Peter R

Wilson S J

Woehl Hubertus

Wolff Sidney C

Wyller Arne A

Yanovitskij Edgard G

Yengibarian Norair

Yorke Harold W

Zahn Jean-Paul 


\title{
Composition of Commission 37
}

\section{Star Clusters \& Associations/Amas stellaires \& associations}

\author{
PRESIDENT: $\quad$ Meylan Georges
}

VICE-PRESIDENT: $\quad$ Sarajedini Ata

\section{ORGANIZING COMMITTEE:}

Cannon Russell D

Castellani Vittorio

Cudworth Kyle McCabe

\section{MEMBERS:}

Aarseth Sverre J

Abou'el-ella Mohamed S

Aiad A Zaki

Alcaino Gonzalo

Alfaro Emilio Javier

Alksnis Andrejs

Allen Christine

Aparicio Antonio

Armandroff Taft E

Auriere Michel

Balazs Bela A

Bell Roger A

Bijaoui Albert

Bosch Guillermo L

Buonanno Roberto

Burkhead Martin S

Butler Dennis

Butler Raymond F

Buzzoni Alberto

Byrd Gene G

Callebaut Dirk K

Caloi Vittoria

Caputo Filippina

Capuzzo Dolcetta Roberto

Carney Bruce William

Carraro Giovanni

Chavarria'k Carlos

Cheng Kwang Ping

Chiosi Cesare S

Christian Carol Ann

Chryssovergis Michael

Chun Mun-suk

Claria Juan

Colin Jacques

Covino Elvira
Da Costa Gary Stewart

Hatzidimitriou Despina Lada

Lada Charles Joseph

Danford Stephen C

D'Antona Francesca

Dapergolas A

Daube-Kurzemniece I A

DeGioia-Eastwood Kathleen

Dehghani Mohammad

Hossein

Demarque Pierre

Demers Serge

Di Fazio Alberto

Dickens Robert $\mathbf{J}$

Dluzhnevskaya Olga B

Drissen Laurent

El Basuny Ahmed Alawy

Elmegreen Bruce Gordon

Fall S Michael

Feinstein Alejandro

Forbes Douglas

Forte Juan Carlos

Friel Eileen D

Fukushige Toshiyuki

Fusi Pecci Flavio

Gascoigne S C B

Geffert Michael

Geisler Douglas P

Giersz Miroslaw

Glushkova Elena V

Golay Marcel

Gratton Raffaela G

Green Elizabeth M

Griffiths William K

Grubissich C

Grundahl Frank

Guetter Harry Hendrik

Hanes David A
Spurzem Rainer

Harris Gretchen L H

Harris Hugh C

Harris William E

Harvel Christopher Alvin

Hassan S M

Hawarden Timothy G

Hazen Martha L

Heggie Douglas C

Henon Michel C

Herbst William

Hesser James E

Heudier Jean-Louis

Hills Jack $G$

Hodapp Klaus-Werner

Hut Piet

Iben Jr Icko

Illingworth Garth D

Ishida Keiichi

Janes Kenneth A

Joshi Umesh C

Kadla Zdenka I

Kamp Lucas Willem

Kandrup Henry Emil

Kilambi G C

Kontizas Evangelos

Kontizas Mary

Kraft Robert P

Kroupa Pavel

Kun Maria

Landolt Arlo U

Lapasset Emilio

Larsson-Leander Gunnar

Laval Annie

Leisawitz David

Leonard Peter James T 


Lloyd Evans Thomas Harry
Lu Phillip K
Lynden-Bell Donald
Maeder Andre
Makino Junichiro
Mardling Rosemary
Markkanen Tapio
Marraco Hugo G
Marshall Kevin P
Martins Donald Henry
Mendez Mariano
Menon T K
Menzies John W
Mermilliod Jean-Claude
Milone Eugene F
Mohan Vijay
Mould Jeremy R
Muminov Muydinjon
Murray C Andrew
Muzzio Juan C
Nemec James
Nesci Roberto
Newell Edward B
Ninkov Zoran
Nissen Poul E
Ogura Katsuo
Ortolani Sergio
Osman Anas Mohamed
Pandey A K
Parsamyan Elma S
Pedreros Mario
Penny Alan John
Peterson Charles John

Petrovskaya Margarita S

Phelps Randy L

Piatti Andres Eduardo

Pilachowski Catherine

Piskunov Anatoly E

Platais Imants $\mathrm{K}$

Poveda Arcadio

Pritchet Christopher J

Renzini Alvio

Richer Harvey B

Richtler Tom

Rountree Janet

Ruprecht Jaroslav

Russeva Tatjana

Sagar Ram

Salukvadze G N

Samus Nikolai N

Sanders Walt L

Santos Jr Joao F

Schild Hansruedi

Schweizer Francois

Semkov Evgeni

Shawl Stephen J

Sher David

Shobbrook Robert R

Shu Chenggang

Simoda Mahiro

Smith Graeme H

Stauffer John Richard

Stetson Peter B

Sugimoto Daiichiro

Suntzeff Nicholas B

Szecsenyi-Nagy Gabor
Takahashi Koji

Terranegra Luciano

Terzan Agop

Thoul Anne A

Tornambe Amedeo

Tripicco Michael J

Trullols I Farreny Enric

Tsuetkov Milcho K

Tsvetkova Katya

Turner David G

Twarog Bruce A

Upgren Arthur R van Altena William $F$

van den Bergh Sidney

VandenBerg Don

Vazquez Ruben Angel

Verschueren Werner

von Hippel Theodore A

Walker Gordon A H

Walker Merle F

Wan Lai

Warren Jr Wayne $\mathrm{H}$

Weaver Harold $\mathbf{F}$

Wehlau Amelia

White Raymond E

Wielen Roland

Wramdemark Stig S

Wu Hsin-Heng

Xiradaki Evangelia

Zakharova Polina E

Zhao Jun Liang

Zinn Robert J 


\section{Composition of Commission 40}

Radio Astronomy/Radioastronomie

PRESIDENT:

VICE-PRESIDENT:
Padrielli Lucia

Rodriguez Luis F

\section{ORGANIZING COMMITTEE:}

Anantharamaiah Kuduvalli R Dewdney Peter E F

Baath Lars B

Berkhuijsen Elly M

Bronfman Leonardo

Davis Richard J

\section{MEMBERS:}

Abdulla Shaker

Abdul Aziz

Ables John G

Ade Peter A R

Aizu Ko

Akabane Kenji A

Akujor Chidi E

Alexander Joseph K

Alexander Paul

Allen Ronald J

Aller Hugh D

Aller Margo $F$

Altenhoff Wilhelm J

Altunin Valery I

Ambrosini Roberto

Andernach Heinz

Aparici Juan

Arnal Marcelo Edmundo

Aschwanden Markus

Assousa George Elias

Aubier Monique G

Aurass Henry

Avery Lorne W

Avignon Yvette

Axon David

Baan Willem A

Baars Jacob W M

Baart Edward E

Bachiller Rafael

Backer Donald Ch

Bagri Durgadas S

Bajaja Esteban

Baker Joanne
Dickel Helene R

Johnston Kenneth J

Litvinenko Leonid N

Marcaide Juan-Maria

Balasubramanian V

Baldwin John E

Balklavs A E

Ball Lewis

Bally John

Balonek Thomas $\mathbf{J}$

Banhatti Dilip Gopal

Barrow Colin H

Bartel Norbert Harald

Barthel Peter

Barvainis Richard

Bash Frank N

Basu Dipak

Batty Michael

Baudry Alain

Baum Stef Alison

Beck Rainer

Benaglia Paula

Benn Chris R

Bennett Charles L

Benson Priscilla J

Benz Arnold O

Berge Glenn L

Bhandari Rajendra

Bhonsle Rajaram V

Bieging John Harold

Biermann Peter L

Biggs James

Bignell R Carl

Biraud Francois

Biretta John Anthony

Birkinshaw Mark

Blair David Gerald
Moran James M

Nan Rendong

Nicolson George D

Schilizzi Richard T

Whiteoak John B

Blandford Roger David

Bloemhof Eric E

Boboltz David A

Bockelee-Morvan Dominique

Booth Roy S

Bos Albert

Bottinelli Lucette

Bowers Phillip F

Bracewell Ronald N

Braude Semion Ya Ag

Breahna Iulian

Bregman Jacob D Ir

Bridle Alan $\mathrm{H}$

Brinks Elias

Britzen Silke H

Broderick John

Broten Norman W

Brouw W N

Browne Ian W A

Bujarrabal Valentin

Burbidge Geoffrey R

Burke Bernard F

Campbell Robert $\mathrm{M}$

Campbell-Wilson Duncan

Carilli Christopher L

Caroubalos C A

Carr Thomas D

Casoli Fabienne

Castets Alain

Caswell James L

Cawthorne Timothy $\mathrm{V}$

Cernicharo Jose

Chan Kwing Lam 
Chandler Claire

Charlot Patrick

Chen Yongjun

Chengalur Jayaram N

Chikada Yoshihiro

Chini Rolf

Cho Se-Hyung

Christiansen Wayne A

Christiansen Wilbur

Chu Hanshu

Chyzy Krzysztof Tadeusz

Clark Barry G

Clark David H

Clark Frank Oliver

Clemens Dan P

Cohen Marshall $\mathrm{H}$

Cohen Raymond $\mathbf{J}$

Cohen Richard S

Cole Trevor William

Coleman Paul Henry

Colomb Fernando R

Colomer Francisco

Combes Francoise

Combi Jorge Ariel

Condon James J

Conklin Edward K

Conway John

Conway Robin G

Cordes James M

Costa Marco E

Cotton Jr William D

Coutrez Raymond A J

Covington Arthur E

Crane Patrick C

Croom David L

Crovisier Jacques

Crutcher Richard M

Cudaback David D

Dagkesamansky Rustam D

Daintree Edward J

Daishido Tsuneaki

Davies Rodney D

Davis Michael M

Davis Robert J

de Bergh Catherine

de Groot T

de Jager Cornelis

de la Noe Jerome

de Ruiter Hans Rudolf

de Vicente Pablo de Young David S

Degaonkar S S

Delannoy Jean

Denisse Jean-Francois

Dent William A

Deshpande Avinash

Despois Didier

Dhawan Vivek

Dickel John $\mathrm{R}$

Dickey John M

Dickman Robert L

Dieter Conklin Nannielou H

Dixon Robert S

Dobashi Kazuhito

Doubinskij Boris A

Dougherty Sean M

Douglas James N

Downes Dennis

Downs George S

Drake Frank D

Drake Stephen A

Dravskikh Alexander F

Dreher John W

Duffett-Smith Peter James

Dulk George A

Dwarakanath K S

Dyson Freeman J

Edelson Rick

Ekers Ronald D

Ellingsen Simon $P$

Ellis G R A

Elsmore Bruce

Emerson Darrel Trevor

Enome Shinzo

Epstein Eugene E

Erickson William C

Eriksen Gunnar

Eshleman Von R

Evans Kenton Dower

Ewing Martin S

Facondi Silvia Rosa

Falcke Heino D

Fanti Roberto

Feigelson Eric D

Feldman Paul A

Felli Marcello

Felten James E

Feretti Luigina

Ferrari Attilio

Fey Alan Lee
Field George B

Finkelstein Andrej M

Fleischer Robert

Florkowski David R

Foley Anthony

Fomalont Edward B

Fort David Norman

Forveille Thierry

Fouque Pascal

Frail Dale Andrew

Franco Mantovani

Frater Robert $\mathrm{H}$

Friberg Per

Frisk Urban

Fuerst Ernst

Fukui Yasuo

Gabuzda Denise C

Gallego Juan Daniel

Galt John A

Garay Guido

Gardner Francis F

Garrett Michael

Garrington Simon

Gaylard Michael John

Geldzahler Bernard J

Gelfreikh Georgij B

Genzel Reinhard

Gerard Eric

Gergely Tomas Esteban

Ghigo Francis D

Gil Janusz A

Gimenez Alvaro

Ginzburg Vitaly L

Gioia Isabella $\mathrm{M}$

Giovannini Gabriele

Gold Thomas

Goldwire Jr Henry C

Gomez Gonzalez Jesus

Gonze Roger F J

Gopalswamy N

Gordon Mark A

Gorgolewski Stanislaw Pr

Gorschkov Alexander G

Gosachinskij Igor V

Goss W Miller

Gottesman Stephen T

Gower J F R

Graham David A

Gratchev Valery

Green Anne 
Green David

Gregorini Loretta

Gregorio-Hetem Jane

Gregory Philip C.

Grewing Michael

Gubchenko Vladimir M

Guelin Michel

Guesten Rolf

Guidice Donald A

Gulkis Samuel

Gull Stephen F

Gupta Yashwant

Gurvits Leonid

Gwinn Carl R

Haddock Fred T

Hagen John P

Hall Peter J

Hamilton P A

Han Jinlin

Han Wenjun

Hanasz Jan

Hanbury Brown Robert

Handa Toshihiro

Hanisch Robert J

Hankins Timothy Hamilton

Hardee Philip

Harnett Julieine

Harris Daniel E

Harten Ronald $\mathrm{H}$

Haschick Aubrey

Hasegawa Tetsuo

Haslam C Glyn T

Hayashi Masahiko

Haynes Martha P

Haynes Raymond F

Hazard Cyril

Heeschen David S

Heiles Carl

Helou George

Henkel Christian

Heske Astrid

Hewish Antony

Hey James Stanley

Hibbard John E

Higgs Lloyd A

Hills Richard E

Hirabayashi Hisashi

Hjalmarson Ake G

Ho Paul T P

Hoang Binh Dy
Hobbs Robert W

Hoegbom Jan A

Hoeglund Bertil

Hofner Peter

Hogg David E

Hollis Jan Michael

Hong Xiaoyu

Howard William E III

Huang Fuquan

Huchtmeier Walter K

Hughes Philip

Hughes Victor A

Hulsbosch A N M

Hunstead Richard W

Ikhsanov Robert N

Ikhsanova Vera $\mathrm{N}$

Inatani Junji

Ipatov Alexander $\mathrm{V}$

Irvine William M

Ishiguro Masato

Iwata Takahiro

Jackson Carole A

Jackson Neal

Jacq Thierry

Jaffe Walter Joseph

Janssen Michael Allen

Jauncey David L

Jenkins Charles R

Jennison Roger $C$

Jewell Philip R

Ji Shuchen

Jin Shenzeng

Johansson Lars Erik B

Johnson Donald R

Joly Francois

Jonas Justin Leonard

Jones Dayton L

Jones Paul

Joshi Mohan N

Jung Jae Hoon

Kaftan May A

Kahlmann Hans Cornelis

Kaidanovski Michael

Kaifu Norio

Kakinuma Takakiyo T

Kalberla Peter

Kameya Osamu

Kandalian Rafik A

Kang Gon Ik

Kardashev Nicolay S
Kasuga Takashi

Kaufmann Pierre

Kawabata Kinaki

Kawabe Ryohei

Kawaguchi Kentarou

Kawamura Akiko

Kazes Ilya

Kellermann Kenneth I

Kenderdine Sidney

Kesteven Michael J 1

Khaikin Vladimir B

Kijak Jaroslaw

Killeen Neil

Kim Hyun-Goo

Kim Kwang-tae

Kim Tu-Whan

Kislyakov Albert G

Klein Karl Ludwig

Klein Ulrich

Ko Hsien C

Kobayashi Hideyuki

Kocharovsky Vladimir V

Kohno Kotaro

Kojima Masayoshi

Konovalenko Olexandr

Korzhavin Anatoly

Kotelnikov Vladimir A

Kramer Michael

Kraus John D

Kreysa Ernst

Krichbaum Thomas $\mathrm{P}$

Krishna Gopal

Krishnamohan S

Krishnan Thiruvenkata

Kronberg Philipp

Kruegel Endrik

Krueger Albrecht

Krygier Bernard

Kuijpers H Jan M E

Kuiper Thomas B H

Kulkarni Prabhakar V

Kulkarni Shrinivas R

Kulkarni Vasant K

Kumkova Irina I

Kundt Wolfgang

Kundu Mukul R

Kuril'chik Vladimir N

Kus Andrzej Jan

Kutner Marc Leslie

Kuzmin Arkadii D 
Kwok Sun

Lada Charles Joseph

Laing Robert

Landecker Thomas L

Lang Kenneth $\mathrm{R}$

Langer William David

Langston Glen I

Lantos Pierre

Large Michael I

LaRosa Theodore $\mathrm{N}$

Lasenby Anthony

Lawrence Charles $\mathrm{R}$

Le Squeren Anne-Marie

Leahy J Patrick

Lee Youngung

Legg Thomas $\mathrm{H}$

Lepine Jacques R D

Lequeux James

Lesch Harald

Lestrade Jean-Francois

Leung Chun Ming

Levreault Russell M

Li Chun-Sheng

Li Gyong Won

Li Hong-Wei

Liang Shiguang

Likkel Lauren Jones

Lilley Edward A

Linke Richard Alan

Lis Dariusz C

Liseau Rene

Little Leslie T

Liu Xiang

Liu Yuying

Lo Kwok-Yung

Locke Jack L

Lockman Felix J

Loiseau Nora

Longair Malcolm S

Loren Robert Bruce

Lovell Sir Bernard

Lozinskaya Tat-yana A

Lubowich Donald A

Luks Thomas

Luo Xianhan

Lyne Andrew $\mathrm{G}$

Macchetto Ferdinando

MacDonald Geoffrey $\mathrm{H}$

MacDonald James

Machalski Jerzy
MacLeod John M

Macrae Donald A

Maehara Hideo

Makoto Inoue

Malofeev Valerij M

Malumian Vigen

Manchester Richard N

Mandolesi Nazzareno

Mao Rui-Qing

Maran Stephen P

Marques Dos Santos $P$

Marscher Alan Patrick

Martin Robert N

Martin-Pintado Jesus

Marvel Kevin B

Masheder Michael

Maslowski Jozef

Masson Colin R

Matheson David Nicholas

Matsakis Demetrios N

Matsuo Hiroshi

Matthews Henry E

Mattila Kalevi

Matveenko Leonid I

Mauersberger Rainer

Maxwell Alan

May J

Mayer Cornell $\mathrm{H}$

McAdam W Bruce

McConnell David

McCulloch Peter M

McKenna Lawlor Susan

McLean Donald J

Mebold Ulrich

Meeks M Littleton

Meier David L

Menon T K

Menten Karl M

Mezger Peter G

Michalec Adam

Miley George $\mathrm{K}$

Mills Bernard Y

Milne Douglas K

Milogradov-Turin Jelena

Mirabel Igor Felix

Mitchell Kenneth J

Miyawaki Ryosuke

Miyoshi Makoto

Mizuno Akira

Moiseev I $G$
Molchanov Andrea $\mathbf{P}$

Montmerle Thierry

Morganti Raffaella

Morimoto Masaki

Morison Ian

Morita Kazuhiko

Morita Koh-ichiro

Moriyama Fumio

Morras Ricardo

Morris David

Morris Mark Root

Moscadelli Luca

Mundy Lee G

Murdoch Hugh S

Mutel Robert Lucien

Muxlow Thomas

Myers Philip C

Nadeau Daniel

Nagnibeda Valery G

Nakajima Junichi

Nakano Takenori

Nasiri Sadollah Gheidari

Neeser Mark J

Neff John S

Nguyen-Quang Rieu

Nicastro Luciano

Nicholls Jennifer

Nishio Masanori

Norris Raymond Paul

O'Dea Christopher P

Oezel Mehmet Emin

Ogawa Hideo

Ohishi Masatoshi

Okoye Samuel E

Okumura Sachiko

Olberg Michael

Onishi Toshikazu

Onuora Lesley Irene

Osterbrock Donald E

O'Sullivan John David

Otmianowska-Mazur

Katarzyna

Owen Frazer Nelson

Pacholczyk Andrzej G

Padman Rachael

Palmer Patrick E

Pankonin Vernon Lee

Papagiannis Michael D

Paredes Jose Maria

Parijskij Yuri N 
Parker Edward A

Parma Paola

Parrish Allan

Pasachoff Jay M

Pauliny Toth Ivan K K

Pauls Thomas Albert

Payne David G

Pearson Timothy J

Pedersen Holger

Pedlar Alan

Peng Bo

Penzias Arno A

Perez Fournon Ismael

Perley Richard Alan

Peters William L III

Pettengill Gordon $\mathrm{H}$

Phillips Thomas Gould

Pick Monique

Planesas Pere

Pogrebenko Sergei

Polatidis Antonios

Ponsonby John E B

Pooley Guy

Porcas Richard

Preston Robert Arthur

Preuss Eugen

Price R Marcus

Priester Wolfgang

Puschell Jeffery John
Qiu Yuhai

Radford Simon John E

Radhakrishnan V

Raimond Ernst

Rao A Pramesh

Raoult Antoinette

Ray Thomas $P$

Razin Vladimir A

Readhead Anthony C S

Reber Grote

Reich Wolfgang

Reid Mark Jonathan

Reif Klaus

Reyes Francisco

Reynolds John

Rhee Myung-Hyun

Ri Jean-Claude

Richer John

Rickard Lee J

Rickett Barnaby James

Riihimaa Jorma J

Riley Julia M

Rioja Maria J

Rizzo Jose Ricardo

Roberts David Hall

Roberts Morton S

Robertson Douglas S

Robertson James Gordon

Robinson Brian J
Robinson Jr Richard D

Roelfsema Peter

Roennaeng Bernt $\mathrm{O}$

Roeser Hans-peter

Roger Robert $S$

Rogers Alan E E

Rogstad David H

Rohlfs Kristen

Romero Gustavo Esteban

Romney Jonathan D

Rowson Barrie

Rubin Robert Howard

Rubio Monica

Rudnick Lawrence

Rudnitskij Georgij M

Russell Jane L

Rydbeck Gustaf H B

Rys Stanislaw

Saikia Dhruba Jyoti

Sakamoto Seiichi

Salpeter Edwin E

Salter Christopher John

Sanamian V A

Sandell Goran Hans 1

Sanders David B

Sargent Anneila I

Sarma N V G 


\section{Composition of Commission 41}

\section{History of Astronomy/Histoire de l'astronomie}

PRESIDENT:

VICE-PRESIDENT:

SECRETARY:
Stephenson F Richard

Gurshtein Alexander A

Orchiston Wayne

\section{ORGANIZING COMMITTEE:}

Dick Wolfgang

Dick Steven J

Kochhar Rajesh K

\section{MEMBERS:}

Ansari S M Razaullah

Aoki Shinko

Badolati Ennio

Bandyopadhyay A

Batten Alan H

Benson Priscilla J

Berendzen Richard

Bessell Michael S

Bishop Roy L

Bo Shuren

Bonoli Fabrizio

Botez Elvira

Brooks Randall C

Brosche Peter

Bruck Mary T

Brunet Jean-Pierre

Carlson John B

Chen Kwan-yu

Chen Meidong

Chinnici Ileana

Corbin Brenda G

Cornejo Alejandro A

Cui Shizhu

Cui Zhenhua

Dadic Zarko

Danezis Emmanuel

de Jong Teije

Debarbat Suzanne V

Deeming Terence J

Dekker E

Devorkin David H

Dewhirst David W

Dobrzycki Jerzy
Nakamura Tsuko

Nha Il-Seong

Sullivan Woodruff T

Warner Brian

Duerbeck Hilmar W

Dumont Simone

Eddy John A

Edmondson Frank K

Ehgamberdiev Shurat

Esteban Lopez Cesara

Fernie J Donald

Firneis Maria G

Florides Petros S

Fodera Seriio Giorgia

Freeman Kenneth C

Freitas Mourao R r

Gingerich Owen

Glass Ian Stewart

Green Daniel William E

Han Wonyong

Hawkins Gerald S

Hayli Avram

Haynes Raymond F

Haynes Roslynn

Hearnshaw John B

Heck Andre

Hemenway Mary Kay M

Herrmann Dieter

Hidayat Bambang

Hirai Masanori

Hockey Thomas Arnold

Huan Nguyen Dinh

Hurukawa Kiitiro

Hysom Edmund J

Hyung Siek

Idlis Grigorij M

Jauncey David L
Jeong Jang Hae

Jiang Xiaoyuan

Jovanovic Bozidar

Khromov Gavriil S

Kiang Tao

Kim Chun Hwey

Kim Yonggi

King David S

King Henry C

Kokott Wolfgang

Krisciunas Kevin

Krupp Edwin C

Kunitzsch Paul

Lang Kenneth R

Launay Francoise

Lee Eun-Hee

Lee Woo-baik

Lee Yong-Sam

Levy Eugene $\mathrm{H}$

Li Zhisen

Liu Ciyuan

Locher Kurt H

Lopes-Gautier Rosaly

Lopez Carlos

Ma Chun-yu

Mathewson Donald S

McAdam W Bruce

McKenna Lawlor Susan

Mickelson Michael E

Mikhail Joseph Sidky

Moesgaard Kristian P

Nadal Robert

Nakayama Shigeru 
Nguyen Mau Tung

Nicolaidis Efthymios

North John David

Oh Kyu Dong

Ohashi Nagayoshi

Ohashi Yukio

Oproiu Tiberiu

Osterbrock Donald E

Papathanasoglou D

Perdrix John

Peterson Charles John

Petri Winfried

Pettersen Bjoern Ragnvald

Pigatto Luisa

Pingree David

Polozhentsev Dimitrij D

Porter Neil A

Poulle Emmanuel

Prokakis Theodore J

Proverbio Edoardo

Pustylnik Izold B

Quan Hejun
Rafferty Theodore J

Reaves Gibson

Ruggles Clive L N

Sbirkova-Natcheva $\mathrm{T}$

Schmadel Lutz D

Shukla K

Signore Monique

Sima Zdislav

Sobouti Yousef

Solc Martin

Soonthornthum Boonrucksar

Stathopoulou Maria

Stavinschi Magdalena

Steinle Helmut

Sterken Christiaan Leo

Stoev Alexei

Sundman Anita

Svolopoulos Sotirios

Swerdlow Noel

Taton Rene

Theodossiou Efstratios

Tobin William
Trimble Virginia L

Vass Gheorghe

Verdet Jean-Pierre

Verdun Andreas $\mathrm{K}$

Volyanskaya Margarita Yu

Wang dechang

Whitaker Ewen A

White Graeme Lindsay

Whiteoak John B

Whitrow Gerald James

Wilkins George A

Wilson Curtis A

$\mathrm{Xi}$ Zezong

Yabuuti Kiyoshi

Yau Kevin K C

Yeomans Donald $\mathrm{K}$

Zhang Peiyu

Zhang Shouzhong

Zhou Yonghong

Zsoldos Endre 


\section{Composition of Commission 42}

\section{Close Binary Stars/Etoiles binaires serrées}

PRESIDENT: Szkody Paula

VICE-PRESIDENT: Gimenez Alvaro

\section{ORGANIZING COMMITTEE:}

Bianchi Luciana

Drechsel Horst

Gies Douglas R

Guinan Edward Francis

Kang Young Woon

\section{MEMBERS:}

Abhyankar Krishna D

Al-Naimiy Hamid M K

Andersen Johannes

Antipova Lyudmila I

Antonopoulou $\mathrm{E}$

Anupama G C

Aquilano Roberto Oscar

Awadalla Nabil Shoukry

Baptista Raymundo

Barone Fabrizio

Bartolini Corrado

Bateson Frank M OBE

Bath Geoffrey T

Batten Alan H

Bell Steven

Blair William $P$

Boffin Henri M J

Bolton C Thomas

Bonazzola Silvano

Bookmyer Beverly B

Bopp Bernard W

Boyle Stephen

Bozic Hrvoje

Bradstreet David H

Brandi Elisande Estela

Breinhorst Robert A

Broglia Pietro

Brownlee Robert R

Bruch Albert

Bruhweiler Jr Fred C

Budding Edwin

Bunner Alan N

Busso Maurizio
Linsky Jeffrey L

Livio Mario

Morrell Nidia

Niemela Virpi S

Callanan Paul

Canalle Joao B G

Catalano Santo

Cester Bruno

Chambliss Carlson R

Chapman Robert D

Chaty Sylvain

Chaubey Uma Shankar

Chen Kwan-yu

Cherepashchuk Analily M

Chochol Drahomir

Choi Kyu-Hong

Cillie G G

Claria Juan

Clausen Jens Viggo

Collins George W ii

Cowley Anne P

Cutispoto Giuseppe

Dadaev Aleksandr N

D'Antona Francesca

de Greve Jean-Pierre

de Groot Mart

de Loore Camiel

Delgado Antonio Jesus

Demircan Osman

Diaz Marcos P

Dorfi Ernst Anton

Dougherty Sean

Doughty Noel A

Duemmler Rudolf

Duerbeck Hilmar W

Dupree Andrea K

Durisen Richard H

\author{
Osaki Toru \\ Rucinski Slavek M \\ Sterken Christiaan Leo \\ Wood Janet $\mathrm{H}$
}

\section{Duschl Wolfgang J}

Eaton Joel A

Edalati Sharbaf Mohammad

Eggleton Peter P

Elias Nicholas

Etzel Paul B

Fabrika Sergei

Faulkner John

Fekel Francis C

Ferluga Steno

Ferrario Lilia

Ferrer Osvaldo Eduardo

Firmani Claudio A

Flannery Brian Paul

Frank Juhan

Fredrick Laurence W

Friedjung Michael

Gallagher III John S.

Garmany Catherine D

Geldzahler Bernard J

Geyer Edward H

Giannone Pietro

Giovannelli Franco

Giuricin Giuliano

Goldman Itzhak

Gosset Eric

Grygar Jiri

Gulliver Austin Fraser

Gunn Alastair G

Gursky Herbert

Guseinov O H

Hadrava Petr

Hakala Pasi J 


\begin{tabular}{|c|c|c|}
\hline Hall Douglas S & Koubsky Pavel & Meliani Mara T \\
\hline Hammerschlag-Hensberge $\mathrm{G}$ & Kraft Robert P & Meyer-Hofmeister Eva \\
\hline Hanawa Tomoyuki & Kraicheva Zdravska & Mezzetti Marino \\
\hline Hantzios Panayiotis & Krautter Joachim & Mikolajewska Joanna \\
\hline Harmanec Petr & Kreiner Jerzy Marek & Mikulasek Zdenek \\
\hline Hassall Barbara J M & Kriz Svatopluk & Milano Leopoldo \\
\hline Haswell Carole A & Kruchinenko Vitaliy G & Milone Eugene F \\
\hline Hazlehurst John & Kruszewski Andrzej & Mineshige Shin \\
\hline Hegedues Tibor & Krzeminski Wojciech & Miyaji Shigeki \\
\hline Heintz Wulff D & Kudashkina Larisa S & Mochnacki Stephan W \\
\hline Hellier Coel & Kumsiashvily Mzia I & Morgan Thomas $\mathrm{H}$ \\
\hline Helt Bodil E & Kurpinska-Winiarska M & Mouchet Martine \\
\hline Hensler Gerhard & Kwee K K & Muerset Urs \\
\hline Herczeg Tibor J & Lacy Claud H & Mumford George S \\
\hline Hilditch Ronald W & Lamb Jr Donald Quincy & Munari Ulisse \\
\hline Hill Graham & Landolt Arlo U & Mutel Robert Lucien \\
\hline Hills Jack G & Lanning Howard Hugh & Nakamura Yasuhisa \\
\hline Holmgren David E & Lapasset Emilio & Nariai Kyoji \\
\hline Holt Stephen S & Larsson Stefan & Nather R Edward \\
\hline Honeycutt R Kent & Larsson-Leander Gunnar & Neff James Edward \\
\hline Horiuchi Ritoku & Lavrov Mikhail I & Nelson Burt \\
\hline Hric Ladislav & Lee Woo-baik & Newsom Gerald $\mathrm{H}$ \\
\hline Hrivnak Bruce J & Lee Yong-Sam & Nha I1-Seong \\
\hline Huang Runqian & Leedjaerv Laurits & Niarchos Panayiotis \\
\hline Hube Douglas P & Leung Kam Ching & Nordstroem Birgitta \\
\hline Hutchings John B & Li Zhongyuan & Norton Andrew $\mathrm{J}$ \\
\hline Ibanoglu Cafir & Linnell Albert P & Oezkan Mustafa Tuerker \\
\hline Imamura James & Liu Qingyao & Oh Kyu Dong \\
\hline Imbert Maurice & Liu Qingzhong & Okazaki Akira \\
\hline Jabbar Sabeh Rhaman & Lloyd Huw & Olah Katalin \\
\hline Jasniewicz Gerard & Lucy Leon B & Oliver John Parker \\
\hline Jeong Jang Hae & Lyuty Victor M & Olson Edward C \\
\hline Joss Paul Christopher & MacDonald James & Osaki Yoji \\
\hline Kadouri Talib Hadi & Maceroni Carla & Paczynski Bohdan \\
\hline Kaitchuck Ronald H & Malasan Hakim Luthfi & Padalia T D \\
\hline Kaluzny Janusz & Mammano Augusto & Pandey Uma Shankar \\
\hline Karetnikov Valentin G R & Mardirossian Fabio & Park Hong Suh \\
\hline Kato Taichi & Maria de Garcia J M & Parthasarathy Mudumba \\
\hline Kawabata Shusaku & Marilli Ettore & Patkos Laszlo \\
\hline Kenny Harold & Marino Brian F & Pavlenko Elena \\
\hline Kenyon Scott J & Markworth Norman Lee & Pavlovski Kresimir \\
\hline Khalesseh Bahram & Marsh Thomas & Peters Geraldine Joan \\
\hline Kim Chun Hwey & Mathieu Robert D & Piccioni Adalberto \\
\hline Kim Ho Il & Mattei Janet Akyuz & Piirola Vilppu E \\
\hline King Andrew $\mathrm{R}$ & Mauder Horst & Plavec Mirek J \\
\hline Kitamura M & Mayer Pavel & Pojmanski Grzegorz \\
\hline Kjurkchieva Diana & Mazeh Tsevi & Polidan Ronald S \\
\hline Koch Robert H & McCluskey Jr George E & Postnov Konstantin A \\
\hline Kolb Ulrich & Meintjes Petrus $\mathrm{J}$ & Potter Stephen B \\
\hline Kondo Yoji & Melia Fulvio & Pringle James E \\
\hline
\end{tabular}


Prokhorov Mikhail E

Pustylnik Izold B

Qiao Guojun

Rafert James Bruce

Rahunen Timo

Rakos Karl D

Ramsey Lawrence W

Rao P Vivekananda

Rasio Frederic A

Refsdal Sjur

Reglero-Velasco Victor

Richards Mercedes T

Ringwald Frederick Arthur

Ritter Hans

Robb Russell M

Robertson John Alistair

Robinson Edward Lewis

Rodono Marcello

Rovithis Peter

Rovithis-Livaniou Helen

Roxburgh Ian W

Ruffert Maximilian

Russo Guido

Sadik Aziz R

Sahade Jorge

Saijo Keiichi

Samec Ronald G

Sanwal N B

Sanyal Ashit

Savonije Gerrit Jan

Scaltriti Franco

Scarfe Colin D

Schiller Stephen

Schmid Hans Martin

Schmidt Hans

Schmidtke Paul C

Schober Hans J

Schoeffel Eberhard F

Seggewiss Wilhelm

Semeniuk Irena
Shafter Allen W

Shakura Nicholaj I

Shaviv Giora

Shen Liangzhao

Shu Frank H

Sima Zdislav

Simmons John Francis 1

Sinvhal Shambhu Dayal

Sion Edward Michael

Sistero Roberto F

Skopal Augustin

Slovak Mark Haines

Smak Joseph I

Smith Robert Connon

Sobieski Stanley

Soderhjelm Staffan

Solheim Jan Erik

Sonti Sreedhar Rao

Sparks Warren M

Srivastava J B

Srivastava Ram Kumar

Stagg Christopher

Starrfield Sumner

Steiman-Cameron Thomas

Steiner Joao E

Stencel Robert Edward

Strohmeier Wolfgang

Sugimoto Daiichiro

Sundman Anita

Svechnikova Maria A

Szafraniec Rozalia

Taam Ronald Everett

Tan Huisong

Tauris Thomas $\mathrm{M}$

Teays Terry $\mathrm{J}$

Todoran Ioan

Tout Christopher

Tremko Jozef

Trimble Virginia L

Turolla Roberto
Tutukov A V

Ureche Vasile

van den Heuvel Edward P J

van Hamme Walter

van Kerkwijk Marten $\mathrm{H}$

van't-Veer Frans

Vaz Luiz Paulo Ribeiro

Vetesnik Miroslav

Vilhu Osmi

Vrielmann Sonja

Wade Richard Alan

Walder Rolf

Walker Richard L

Walker William S G

Ward Martin John

Warner Brian

Webbink Ronald $\mathrm{F}$

Weiler Edward J

Wellmann Peter

Wheeler J Craig

White II James Clyde

Williamon Richard M

Williams Glen A

Williams Robert E

Wilson Robert E

Yamaoka Hitoshi

Yamasaki Atsuma

Yoon Tae

Zakirov Mamnum

Zeilik Michael Ii

Zhang Er-Ho

Zhang Jintong

Zhou Daoqi

Zhou Hongnan

Ziolkowski Janusz

Zola Stanislaw

Zuiderwijk Edwardus J

Zwitter Tomaz 


\section{Composition of Commission 44}

Space \& High Energy Astrophysics/Astrophysique spatiale \& des hautes énergies

PRESIDENT: $\quad$ Srinivasan G

VICE-PRESIDENT: Okuda Haruyuki

\section{ORGANIZING COMMITTEE:}

Baliunas Sallie L

Brosch Noah

Cesarsky Catherine J

Courvoisier Thierry J-L

da Costa Jose Marques

Domingo Vicente

Fabian Andrew C

\section{MEMBERS:}

Abramowicz Marek

Acharya Bannanje S

Acton Loren W

Adams David J

Agrawal P C

Aguiar Odylio Denys

Ahluwalia Harjit Singh

Ahmad Imad Aldean

Aizu Ko

Alexander Joseph K

Almleaky Yasseen

Apparao K M V

Arafune Jiro

Arnaud Monique

Arnould Marcel L

Arons Jonathan

Aschenbach Bernd

Asseo Estelle

Audouze Jean

Awaki Hisamitsu

Axford W Ian

Ayres Thomas R

Baan Willem A

Badiali Massimo

Barstow Martin Adrian

Basu Dipak

Baym Gordon Alan

Becker Robert Howard

Begelman Mitchell Craig

Belloni Tomaso

Benedict George F
Fazio Giovanni G

Fransson Claes

Inoue Hajime

Li Zhongyuan

O'Brien Paul Thomas

Oertel Goetz K

Quintana Hernan
Rengarajan $\mathrm{T} \mathrm{N}$

Schilizzi Richard T

Shustov Boris M

Thronson Jr Harley Andrew

Vilhu Osmi

Wamsteker Willem

Wang Zhenru

Bowyer C Stuart

Boyarchuk Alexander A

Boyd Robert L F

Bradley Arthur J

Braga Joao

Brandt John C

Brandt Soeren K

Brecher Kenneth

Breslin Ann

Brinkman Bert C

Brown Alexander

Bruhweiler Jr Fred C

Bruner Marilyn E

Bumba Vaclav

Bunner Alan N

Burbidge Geoffrey R

Burger Marijke

Burke Bernard $\mathrm{F}$

Burrows Adam Seth

Burrows David Nelson

Burton William M

Butler C John

Butterworth Paul

Camenzind Max

Cameron Alastair G W

Campbell Murray F

Cardini Daniela

Carpenter Kenneth $G$

Carroll P Kevin

Carver John H

Cash Jr Webster C 
Casse Michel

Catura Richard C

Cavaliere Alfonso $\mathrm{G}$

Chakrabarti Sandip K

Chakraborty Deo K

Chapman Robert D

Charles Philip Allan

Chechetkin Valerij M

Cheng Kwong-sang

Chengmo Zhang

Cheung Cynthia Y

Chian Abraham Chian-Long

Chikawa Michiyuki

Chitre Shashikumar M

Chochol Drahomir

Chubb Talbot A

Chupp Edward L

Churazov Eugene $M$

Clark George W

Clark Thomas Alan

Clay Roger

Code Arthur D

Cohen Jeffrey $M$

Collin-Souffrin Suzy

Comastri Andrea

Condon James J

Corbet Robin Henry D

Corcoran Michael Francis

Cordova France A D

Courtes Georges

Cowie Lennox Lauchlan

Cowsik Ramanath

Crannell Carol Jo

Cruise Adrian Michael

Culhane Leonard

Curir Anna

Cusumano Giancarlo

da Costa Antonio A

Dadhich Naresh

Dai Zigao

D'Amico Flavio

Damle S V

Dautcourt G

Davidsen Arthur Falnes

Davidson William

Davis Michael M

Davis Robert J

Davis Jr Leverett

Dawson Bruce

de Felice Fernando de Jager Cornelis

de Martino Domitilla

de Young David S

Debrunner Hermann

Della Ceca Roberto

Dempsey Robert C

Dennis Brian Roy

Dermer Charles Dennison

Dewitt Bryce S

Di Cocco Guido

Digel Seth William

Disney Michael J

Dokuchaev Vyacheslav I

Dolan Joseph F

Dotani Tadayasu

Downes Turlough

Drake Frank D

Drury Luke O'Connor

Dunkelman Lawrence

Duorah Hira Lal

Dupree Andrea K

Durouchoux Philippe

Duthie Joseph G

Edelson Rick

Eichler David

Eilek Jean

El Raey Mohamed E

Elvis Martin S

Emanuele Alessandro

Evans W Doyle

Fabricant Daniel G

Fang Li-zhi

Faraggiana Rosanna

Feldman Paul Donald

Felten James E

Fender Robert $P$

Fenton K B

Ferrari Attilio

Fichtel Carl E

Field George B

Fisher Philip C

Fishman Gerald J

Fitton Brian

Foing Bernard $\mathrm{H}$

Fomin Valery

Forman William Richard

Franceschini Alberto

Frandsen Soeren

Frank Juhan

Fredga Kerstin
Frisk Urban

Fu Chengqi

Furniss Ian

Gabriel Alan H

Gaisser Thomas K

Galeotti Piero

Garcia Howard A

Garmire Gordon P

Gehrels Neil

Georgantopoulos Ioannis

Geppert Ulrich R M E

Gezari Daniel Ysa

Ghisellini Gabriele

Giacconi Riccardo

Gilra Daya P

Ginzburg Vitaly L

Gioia Isabella $M$

Glaser Harold

Gold Thomas

Goldsmith Donald W

Goldwurm Andrea

Gondhalekar Prabhakar

Gonzales'a Walter D

Grebenev Sergei A

Greenhill John

Greisen Kenneth I

Grenier Isabelle

Grewing Michael

Greyber Howard D

Griffiths Richard E

Grindlay Jonathan E

Gull Theodore R

Gunn James E

Gursky Herbert

Guseinov O H

Hack Margherita

Haddock Fred T

Hakkila Jon Eric

Hall Andrew Norman

Hallam Kenneth L

Hameury Jean-Marie

Han Zhengzhong

Hang Hengrong

Hannikainen Diana C

Hardcastle Martin J

Harms Richard James

Harris Daniel E

Harvey Christopher C

Harvey Paul Michael

Harwit Martin 
Hasinger Guenther

Hatsukade Isamu

Haubold Hans Joachim

Hauser Michael G

Hawkes Robert Lewis

Hawking Stephen W

Hawkins Isabel

Haymes Robert C

Hearn Anthony G

Heckathorn Harry M

Hein Righini Giovanna

Heise John

Helfand David John

Helmken Henry F

Helou George

Henoux Jean-Claude

Henriksen Richard N

Henry Richard C

Hensberge Herman

Heske Astrid

Hoffman Jeffrey Alan

Holberg Jay B

Holloway Nigel J

Holt Stephen S

Houziaux Leo

Howarth Ian Donald

Hoyle Fred

Hoyng Peter

Hu Wenrui

Huang Keliang

Huang Yong-Feng

Huber Martin C E

Hunt Leslie

Hurley Kevin C

Hutchings John B

Ichimaru Setsuo

Imamura James

Imhoff Catherine L

Ipser James $\mathrm{R}$

Ishida Manabu

Israel Werner

Ito Kensai A

Itoh Masayuki

Iyengar K V K

Jackson John Charles

Jaffe Walter Joseph

Jamar Claude A J

Janka Hans Thomas

Jaranowski Piotr

Jenkins Edward B
Jokipii J R

Jones Frank Culver

Jones Thomas Walter

Jordan Carole

Jordan Stuart D

Joss Paul Christopher

Juliusson Einar

Kafatos Minas

Kahabka Peter

Kaneda Hidehiro

Kaper Lex

Kapoor Ramesh Chander

Kasturirangan $\mathrm{K}$

Katz Jonathan I

Kawai Nobuyuki

Kellermann Kenneth I

Kellogg Edwin M

Kembhavi Ajit K

Kessler Martin F

Kii Tsuneo

Killeen Neil

Kim Yonggi

Kimble Randy A

Kirk John

Klinkhamer Frans

Klose Sylvio

Kobayashi Shiho

Kocharov Grant E

Kocharovsky Vitaly V

Koch-Miramond Lydie

Koide Shinji

Kojima Yasufumi

Kolb Edward W

Kondo Masaaki

Kondo Yoji

Koshiba Masa-Toshi

Koupelis Theodoros

Koyama Katsuji

Kozlowski Maciej

Kozma Cecilia A

Kraushaar William L

Kreisel E

Kristiansson Krister

Kryvdyk Volodymyr G

Kuiper Lucien

Kulsrud Russell M

Kumagai Shiomi

Kundt Wolfgang

Kurt Vitaliy G

Kusunose Masaaki
Lamb Frederick K

Lamb Susan Ann

Lamb Jr Donald Quincy

Lamers Henny J G L M

Lampton Michael

Lasher Gordon Jewett

Lattimer James $M$

Lea Susan Maureen

Leckrone David S

Leighly Karen Marie

Lemaire Philippe

Lewin Walter H G

Li Tipei

Li Yuanjie

Li Zongwei

Liang Edison $P$

Lindblad Bertil A

Linsky Jeffrey L

Linsley John

Lochner James Charles

Long Knox S

Longair Malcolm S

Lovelace Richard V E

Lovell Sir Bernard

Lu Jufu

Lu Tan

Luest Reimar

Luminet Jean-Pierre

Lynden-Bell Donald

Ma YuQian

Maccacaro Tommaso

Macchetto Ferdinando

Maggio Antonio

Makarov Valeri

Makoto Inoue

Malaise Daniel J

Malitson Harriet $\mathrm{H}$

Malkan Matthew Arnold

Manara Alessandro A

Mandolesi Nazzareno

Maran Stephen P

Marar T M k

Marov Mikhail Ya

Martin Inacio Malmonge

Masai Kuniaki

Mason Glenn M

Mason Keith Owen

Mather John Cromwell

Matsumoto Ryoji

Matsuoka Masaru 


Matz Steven Micheal
Mazurek Thaddeus John
McBreen Brian Philip
McCluskey Jr George E
McCray Richard
McWhirter R W Peter
Mead Jaylee Montague
Medina Jose
Medina Tanco Gustavo A
Meier David L
Meiksin Avery Abraham
Melia Fulvio
Melnick Gary J
Melrose Donald B
Mestel Leon
Meszaros Peter
Mewe R
Meyer Friedrich
Meyer Jean-Paul
Micela Giuseppina
Michel F Curtis
Miller Guy Scott
Miller John C
Miller Michael C
Mineo Teresa
Miyaji Shigeki
Miyamoto Sigenori
Mizumoto Yoshihiko
Mizutani Kohei
Modisette Jerry L
Monet David G
Monfils Andre G
Montmerle Thierry
Moon Shin Haeng
Moos Henry Warren
Morgan Thomas H
Morrison Philip
Morton Donald C
Motch Christian
Murakami Hiroshi
Murakami Toshio
Murdock Thomas Lee
Murtagh Fionn
Murthy Jayant
Naidenov Victor O
Nakayama Kunji
Neeman Yuval
Neff Susan Gale
Ness Norman F
Neupert Werner M

Matz Steven Micheal

Mazurek Thaddeus John

McBreen Brian Philip

McCluskey Jr George

McCray Richard

McWhirter R W Peter

Mead Jaylee Montague

Medina Jose

Medina Tanco Gustavo A

Meier David L

Meiksin Avery Abraham

Melia Fulvio

Melnick Gary J

Mestel Leon

Meszaros Peter

Mewe $\mathrm{R}$

Meyer Friedrich

Meyer Jean-Paul

Micela Giuseppina

Michel F Curtis

Miller Guy Scott

Mineo Teresa

Miyaji Shigeki

Miyamoto Sigenori

Mizumoto Yoshihiko

Mizutani Kohei

Modisette Jerry L

Monet David G

onfils Andre $\mathrm{G}$

Moos Henry Warren

Morgan Thomas $\mathrm{H}$

Morrison Philip

Morton Donald C

Motch Christian

Murakami Hiroshi

Murakami Toshio

Murdock Thomas Lee

Murtagh Fionn

Murthy Jayant

Naidenov Victor O

Nakayama Kunji

Neeman Yuval

Neff Susan Gale

Neupert Werner M
Nichols-Bohlin Joy

Nicollier Claude

Nityananda Ram

Nomoto Ken'ichi

Norci Laura

Nordh Lennart H

Norman Colin A

Novick Robert

Noyes Robert W

Nulsen Paul

O'Connell Robert F

Oda Minoru

Oda Naoki

Oezel Mehmet Emin

Ogawara Yoshiaki

Ogelman Hakki B

Okeke Pius N

Okoye Samuel E

Okuda Toru

Olthof Hindericus

O'Mongain Eon

Oohara Ken-ichi

Orio Marina

Osborne Julian P

Ostriker Jeremiah $\mathrm{P}$

Ostrowski Michal

O'Sullivan Denis F

Owen Tobias C

Ozernoy Leonid $\mathrm{M}$

Pacholczyk Andrzej G

Paciesas William S

Pacini Franco

Page Clive G

Paltani Stephane

Palumbo Giorgio G C

Pandey Uma Shankar

Papagiannis Michael D

Park Myeong-gu

Parker Eugene N

Parkinson John $\mathrm{H}$

Parkinson William $\mathrm{H}$

Pauliny Toth Ivan K K

Peacock Anthony

Peng Qiuhe

Perola Giuseppe C

Perry Peter M

Peters Geraldine Joan

Peterson Bruce A

Peterson Laurence E

Pethick Christopher J
Petkaki Panagiota

Petro Larry David

Petrosian Vahe

Phillips Kenneth J H

Piddington Jack H Res Fel

Pinkau K

Pinto Philip Alfred

Pipher Judith L

Piro Luigi

Polidan Ronald S

Porter Neil A

Pounds Kenneth A

Poutanen Juri

Prasanna A R

Preuss Eugen

Price Stephan Donald

Proszynski Mieczyslaw

Protheroe Raymond J

Prusti Timo

Qiu Yulei

Qu Qinyue

Radhakrishnan V

Ramadurai Souriraja

Rao Arikkala Raghurama

Rao Ramachandra V

Raubenheimer Barend C

Razdan Hiralal

Rees Martin J

Reeves Edmond M

Reeves Hubert

Reichert Gail Anne

Reig Pablo

Rense William A

Rhoads James

Riegler Guenter R

Roman Nancy Grace

Rosendhal Jeffrey D

Rosner Robert

Rovero Adrian Carlos

Ruben Gerhard

Ruder Hanns

Ruffini Remo

Sabau-Graziati Lola

Sagdeev Roald Z

Sahade Jorge

Salpeter Edwin E

Salvati Marco

Samimi Jalal

Sanchez Norma G

Sanders Wilton Turner III 
Santos Nilton Oscar

Sartori Leo

Saslaw William C

Sato Katsuhiko

Savage Blair D

Savedoff Malcolm P

Scargle Jeffrey D

Schaefer Gerhard

Schatten Kenneth $\mathrm{H}$

Schatzman Evry

Scheuer Peter A G

Schmidt K H

Schmitt Juergen H M M

Schnopper Herbert W

Schoeneich W

Schreier Ethan J

Schultz G V

Schwartz Daniel A

Schwartz Steven Jay

Schwehm Gerhard

Sciortino Salvatore

Scott John S

Seielstad George A

Selvelli Pierluigi

Sequeiros Juan

Setti Giancarlo

Seward Frederick D

Shaham Jacob

Shakhov Boris A

Shakura Nicholaj I

Shapiro Maurice M

Shaver Peter A

Shaviv Giora

Sheffield Charles

Shibai Hiroshi

Shibazaki Noriaki

Shields Gregory A

Shigeyama Toshikazu

Shivanandan Kandiah

Shukre C S

Signore Monique

Sikora Marek

Silberberg Rein

Silvestro Giovanni

Simon Paul C

Simon Vojtech

Singh Kulinder Pal

Singh Markandey

Skilling John

Smale Alan Peter
Smith Barham W

Smith Bradford A

Smith Linda J

Smith Peter L

Snow Theodore $\mathbf{P}$

Sofia Sabatino

Sokolov Vladimir V

Somasundaram Seetha

Sonneborn George

Sood Ravi

Spada Gianfranco

Speer R J

Sreekumar Parameswaran

Srivastava Dhruwa

Stachnik Robert V

Staubert Ruediger Prof

Stecher Theodore P

Stecker Floyd W

Steigman Gary

Steiner Joao E

Stencel Robert Edward

Stepanian A A

Stephens S A

Stern Robert Allan

Stevens Ian

Stier Mark T

Stockman Jr Hervey S

Stone R G

Straumann Norbert

Stringfellow Guy Scott

Strohmayer Tod E

Strong Ian B

Sturrock Peter A

Sun Wei-Hsin

Sunyaev Rashid A

Suzuki Hideyuki

Svensson Roland

Swank Jean Hebb

Tagliaferri Gianpiero

Takahara Fumio

Takahash Tadayuki

Takahashi Masaaki

Takakura Tatsuo Emer

Tanaka Yasuo

Tanaka Yasuo

Tashiro Makoto

Terrell Jr Nelson James

Thomas Roger $\mathbf{J}$

Thorne Kip S

Tomimatsu Akira
Totsuka Yoji

Tovmassian Hrant $M$

Traub Wesley Arthur

Trimble Virginia L

Truemper Joachim

Truran Jr James W

Trussoni Edoardo

Tsunemi Hiroshi

Tsuru Takeshi

Tsuruta Sachiko

Tsygan Anatolii I

Tylka Allan J

Underhill Anne B

Underwood James $\mathrm{H}$

Upson Walter L II

Vahia Mayank N

Valnicek Boris

Valtonen Mauri J

van Beek Frank

van den Heuvel Edward P J

van der Hucht Karel A

van der Walt D J

van Duinen $R \mathbf{J}$

van Putten Maurice

van Riper Kenneth A

van Speybroeck Leon $P$

Vial Jean-Claude

Vidal Nissim V

Vidal-Madjar Alfred

Viollier Raoul D

Viotti Roberto

Voelk Heinrich J

Vrtilek Saeqa Dil

Walker Helen J

Walsh Dennis

Wanas Mamdouh Ishaac

Wang Shouguan

Wang Shui

Wang Yi-ming

Warner John W

Weaver Kimberly A

Weaver Thomas A

Webster Adrian S

Wehrle Ann Elizabeth

Wei Daming

Weiler Edward $\mathbf{J}$

Weiler Kurt W

Weinberg J L

Weisheit Jon C

Weisskopf Martin Ch 
Wells Donald C

Wentzel Donat G

Wesselius Paul R

Westfold Kevin C

Westphal James A

Wheeler J Craig

Wheeler John A

White Nicholas Ernest

Will Clifford M

Willis Allan J

Willner Steven Paul

Wilson Andrew S

Wilson James $R$

Wilson Robert

Winkler Christoph

Wolfendale Arnold W
Wolstencroft Ramon D

Wolter Anna

Woltjer Lodewijk

Worrall Diana Mary

Wray James D

Wu Chi Chao

Wu Xuejun

Wunner Guenter

Yadav Jagdish Singh

Yamada Shoichi

Yamamoto Yoshiaki

Yamasaki Tatsuya

Yamashita Kojun

Yamauchi Makoto

Yamauchi Shigeo

Yang Lantian
Yang Pibo

Yi Insu

Yock Philip

Yoshida Atsumasa

You Junhan

Zamorani Giovanni

Zarnecki John Charles

Zdziarski Andrzej

Zhang Heqi

Zhang Jialu

Zhang William W

Zhang Zhen-Jiu

Zheng Wei

Zombeck Martin V

Zou Huicheng 
Composition of Commission 45

Stellar Classification/Classification stellaire

PRESIDENT: $\quad$ Lloyd Evans Thomas Harry

VICE-PRESIDENT: Corbally Christopher

\section{ORGANIZING COMMITTEE:}

Drilling John $\mathrm{S}$

Gerbaldi Michele

Giridhar Sunetra

Humphreys Roberta M

\section{MEMBERS:}

Albers Henry

Ardeberg Arne L

Arellano Ferro Armando

Babu G S D

Baglin Annie

Barbier-Brossat Madeleine

Bartaya R A

Bartkevicius Antanas

Bell Roger A

Bidelman William $P$

Blanco Victor $M$

Buscombe William

Buser Roland

Celis Leopoldo

Cester Bruno

Cherepashchuk Analily M

Christy James Walter

Claria Juan

Coluzzi Regina

Cowley Anne P

Crawford David L

Divan Lucienne

Duflot Marcelle

Egret Daniel

Faraggiana Rosanna

Feast Michael W

Fehrenbach Charles

Feltzing Sofia

Fitzpatrick Edward L

Fukuda Ichiro

Garmany Catherine D

Garrison Robert $F$

Geyer Edward $\mathrm{H}$

Glagolevskij Juri V
Luri Xavier

Pasinetti Laura E

Straizys V

Golay Marcel

Gray Richard O

Grenon Michel

Guetter Harry Hendrik

Gupta Ranjan

Gurzadian Grigor A

Hack Margherita

Hallam Kenneth L

Hauck Bernard

Hayes Donald S

Houk Nancy

Huang Lin

Kato Ken-ichi

Kharadze E K

Kurtanidze Omar

Kurtz Donald Wayne

Kurtz Michael Julian

Labhardt Lukas

Lasala Gerald J

Lattanzio John

Lee Sang Gak

Levato Orlando Hugo

Loden Kerstin R

Low Frank J

Lu Phillip K

Lutz Julie $\mathrm{H}$

MacConnell Darrell J

Maehara Hideo

Malagnini Maria Lucia

Malaroda Stella M

McClure Robert D

Mcnamara Delbert H

Mead Jaylee Montague

Mendoza V Eugenio E von Hippel Theodore A

Weiss Werner W

Zdanavicius Kazimeras

Morossi Carlo

Morrell Nidia

Nicolet Bernard

North Pierre

Notni P

Oja Tarmo

Olsen Erik H

Osborn Wayne

Oswalt Terry D

Parsons Sidney B

Perry Charles L

Philip A G Davis

Pizzichini Graziella

Preston George W

Rautela B S

Roman Nancy Grace

Rountree Janet

Rudkjobing Mogens

Sanwal N B

Schild Rudolph E

Schmidt-Kaler Theodor

Seitter Waltraut C

Sharpless Stewart

Shore Steven N

Shvelidze Teimuraz D

Sinnerstad Ulf E

Sion Edward Michael

Sonti Sreedhar Rao

Steinlin Uli

Stephenson C Bruce

Stock Jurgen D

Strobel Andrzej

Upgren Arthur R

Walborn Nolan R 
Walker Gordon A H

Warren Jr Wayne $\mathrm{H}$

Weaver William Bruce

Wesselius Paul R
Westerlund Bengt E

Williams John A

Wing Robert $\mathrm{F}$

Wu Hsin-Heng
Wyckoff Susan

Yamashita Yasumasa

Yoss Kenneth M 


\section{Composition of Commission 46}

Astronomy Education \& Development/Education \& Développement en astronomie

PRESIDENT: $\quad$ Isobe Syuzo

VICE-PRESIDENT: $\quad$ Pasachoff Jay M

ORGANIZING COMMITTEE:

Batten Alan $\mathrm{H}$

Fierro Julieta

Gerbaldi Michele

MEMBERS:

Acker Agnes

Aguilar Maria Luisa

Aiad A Zaki

Alexandrov Yuri V

Al-Naimiy Hamid M K

Al-sabti Abdul Adim

Alvarez-Pomares Oscar A

Anandaram Mandayam N

Andersen Johannes

Andrews Frank

Ansari S M Razaullah

Arellano Ferro Armando

Aubier Monique $\mathrm{G}$

Bajaja Esteban

Barthel Peter

Benson Priscilla J

Bernabeu Guillermo

Bjornsson Claes-Ingvar

Black Adam Robert S

Bobrowsky Matthew

Bochonko D Richard

Borchkhadze Tengiz M

Botez Elvira

Bottinelli Lucette

Boyarchuk Alexander A

Braes L L E

Brieva Eduardo

Brosch Noah

Bruck Mary T

Budding Edwin

Buscombe William

Caccin Bruno

Calvet Nuria

Cannon Wayne $\mathrm{H}$

Capaccioli Massimo
Jones Barrie W

Percy John R

Tolbert Charles R

Carter Brian

Catala Poch M A

Celebre Cynthia P

Chamberlain Joseph M

Chamcham Khalil

Chitre Dattakumar M

Clarke David

Codina Landaberry Sayd J

Corbally Christopher

Cottrell Peter Ledsam

Couper Heather Miss

Craig Nahide

Crawford David L

Cui Zhenhua

Daniel Jean-Yves

DeGioia-Eastwood Kathleen

Demircan Osman

Ducati Jorge Ricardo

Dupuy David L

Duval Marie-France

Dworetsky Michael M

El Eid Mounib

Fairall Anthony $\mathbf{P}$

Fernandez Julio A

Fernandez-Figueroa M J

Fienberg Richard $\mathrm{T}$

Fleck Robert Charles

Florsch Alphonse

Forbes Douglas

Gallino Roberto

Ghobros Roshdy Azer

Gill Peter B J

Gimenez Alvaro

Gingerich Owen

Gouguenheim Lucienne
Wentzel Donat G

West Richard M

Gray Richard O

Gregorio-Hetem Jane

Guinan Edward Francis

Gurm Hardev S

Hafizi Mimoza

Haubold Hans Joachim

Haupt Hermann F

Havlen Robert J

Hawkins Isabel

Haywood J

Hearnshaw John B

Hemenway Mary Kay M

Heudier Jean-Louis

Hidayat Bambang

Hockey Thomas Arnold

Hoff Darrel Barton

Houziaux Leo

Huan Nguyen Dinh

Huang Tianyi

Ilyas Mohammad

Impey Christopher D

Inglis Michael

Iwaniszewska Cecylia

Jarrett Alan H

Jorgensen Henning E

Karetnikov Valentin G R

Keller Hans Ulrich

Kiasatpour Ahmad

Kitchin Christopher R

Kolka Indrek

Kononovich Edward V

Kourganoff Vladimir

Kozai Yoshihide

Kreiner Jerzy Marek

Krishna Gopal 
Krupp Edwin C

Lago Maria Teresa V T

Lai Sebastiana

Lanciano Nicoletta

Leung Chun Ming

Leung Kam Ching

Li Zongwei

Little-Marenin Irene $\mathbf{R}$

Lomb Nicholas Ralph

Luck John M

$\mathrm{Ma} \mathrm{Er}$

Ma Xingyuan

Maciel Walter J

Macrae Donald A

Maddison Ronald $\mathrm{Ch}$

Malasan Hakim Luthfi

Mamadazimov Mamadmuso

Marsh Julian C D

Martinet Louis

Martinez Peter

Mavridis L N

Maza Jose

McNally Derek

Meidav Meir

Milogradov-Turin Jelena

Mizuno Takao

Moreels Guy

Morimoto Masaki

Muzzio Juan C

Najid Nour-Eddine

Narlikar Jayant V

Nguyen-Quang Rieu

Nha Il-Seong

Nicolov Nikolai S

Nicolson Iain

Ninkovic Slobodan

Noels Arlette

Norton Andrew J

Oja Heikki

Okeke Pius N

Okoye Samuel E
Olsen Fogh H J

Onuora Lesley Irene

Osborn Wayne

Osorio Jose J S P

Oswalt Terry D

Othman Mazlan

Owaki Naoaki

Pandey Uma Shankar

Parisot Jean-Paul

Penston Margaret

Pineda de Carias Maria

Cristina

Pokorny Zdenek

Ponce G A

Prabhakaran Nayar S R

Proverbio Edoardo

Quamar Jawaid

Querci Francois R

Raboud Didier

Raghavan Nirupama

Ramadurai Souriraja

Rijsdijk Case

Robbins R Robert

Roberts Morton S

Robinson Leif J

Ros Rosa M

Rosenzweig-Levy Patrica

Roslund Curt

Routly Paul M

Roy Archie E

Sadat Rachida

Safko John L

Sahade Jorge

Sanahuja Blas

Sandqvist Aage

Sattarov Isroil

Saxena P P

Sbirkova-Natcheva $T$

Schleicher David G

Schlosser Wolfhard

Schmitter Edward F
Schroeder Daniel J

Seeds Michael August

Shen Chun-Shan

Shipman Harry L

Smith Francis Graham

Solheim Jan Erik

Soriano Jr. Bernado M

Stefl Vladimir

Stenholm Bjoern

Stoev Alexei

Straizys V

Sukartadiredja Darsa

Sutantyo Winardi

Svestka Jiri

Swarup Govind

Szecsenyi-Nagy Gabor

Szostak Roland

Taborda Jose Rosa

Torres-Peimbert Silvia

Troche-Boggino A E

Tsubota Yukimasa

van den Heuvel Edward P J

van Santvoort Jacques

Vauclair Sylvie D

Vujnovic Vladis

Wang Shouguan

Wang Shunde

Ward Richard A

White II James Clyde

Whitelock Patricia Ann

Williamon Richard M

Willmore A Peter

Woo Jong Ok

Ye Shuhua

Zakirov Mamnum

Zealey William $J$

Zeilik Michael Ii

Zhao Jun Liang

Zimmermann Helmut 


\section{Composition of Commission 47}

\section{Cosmology/Cosmologie}

PRESIDENT:

VICE-PRESIDENT:
Peacock John Andrew

Lilly Simon J

\section{ORGANIZING COMMITTEE:}

Campusano Luis E
da Costa Luiz A N
Ellis George F R

MEMBERS:

Adams Jenni

Aizu Ko

Alimi Jean-Michel A

Allan Peter M

Amendola Luca

Andreani Paola Michela

Audouze Jean

Auluck Faqir Chand

Azuma Takahiro

Bahcall Neta A

Bajtlik Stanislaw

Baldwin John E

Banday Anthony $\mathrm{J}$

Banerji Sriranjan

Banhatti Dilip Gopal

Barberis Bruno

Barbuy Beatriz

Bardeen James M

Bardelli Sandro

Barrow John David

Bartelmann Matthias

Barthel Peter

Basu Dipak

Bechtold Jill

Beckman John E

Beesham Aroonkumar

Belinsky Vladimir A

Bennett Charles L

Bergeron Jacqueline A

Bergstroem Lars

Berman Marcelo S

Bertola Francesco

Bertschinger Edmund

Betancort-Rijo Juan
Koo David C-Y

Le Fevre Olivier

Suto Yasushi

Bhavsar Suketu $P$

Bicknell Geoffrey V

Bignami Giovanni F

Birkinshaw Mark

Biviano Andrea

Blanchard Alain

Bleyer Ulrich

Bludman Sidney A

Boksenberg Alec

Bond John Richard

Bondi Hermann

Bonnor W B

Borgani Stefano

Bouchet Francois R

Boyle Brian

Brecher Kenneth

Burbidge Geoffrey R

Burns Jr Jack O'Neal

Calvani Massimo

Cappi Alberto

Carr Bernard John

Castagnino Mario

Cavaliere Alfonso $\mathrm{G}$

Cesarsky Diego A

Chang Kyongae

Charlot Stephane

Chen Da-Ming

Cheng Fuhua

Cheng Fuzhen

Chen Jiansheng

Chincarini Guido L

Chitre Dattakumar M

Chodorowski Michal

Chu Yaoquan

Claria Juan
Szalay Alex

Webster Rachel

Clowes Roger $\mathrm{G}$

Cocke William John

Cohen Jeffrey M

Cohen Ross D

Coles Peter

Colless Matthew

Colombi Stephane

Condon James J

Cora Sofia Alejandra

Courteau Stephane

Crane Patrick C

Crane Philippe

Cristiani Stefano

Da Costa Gary Stewart

Dadhich Naresh

Danese Luigi

Das P K

Davidson William

Davies Paul Charles W

Davies Roger L

Davis Marc

Davis Michael M

de Lapparent-Gurriet Valerie

de Ruiter Hans Rudolf

de Zotti Gianfranco

Dekel Avishai

Dell'Antonio P Ian

Demianski Marek

Dhurandhar Sanjeev

Dionysiou Demetrios

Djorgovski Stanislav

Doroshkevich Andrei G

Dressler Alan

Drinkwater Michael J

Dultzin-Hacyan D 
Dunlop James

Dunsby Peter

Dyer Charles Chester

Efstathiou George

Ehlers Jurgen

Einasto Jaan

Ellis Richard S

Elvis Martin S

Enginol Turan B

Faber Sandra M

Fairall Anthony $\mathbf{P}$

Falk Jr Sydney W

Fall S Michael

Fang Li-zhi

Felten James E

Feng Long Long

Field George B

Filippenko Alexei V

Firmani Claudio A

Florides Petros S

Focardi Paola

Fomin Piotr Ivanovich

Fong Richard

Ford Holland C Res

Forman William Richard

Fouque Pascal

Franceschini Alberto

Frenk Carlos S

Fujimoto Mitsuaki

Fukugita Masataka

Fukui Takao

Galletto Dionigi

Garilli Bianca

Garrison Robert F

Geller Margaret Joan

Giallongo Emanuele

Gioia Isabella M

Giuricin Giuliano

Gold Thomas

Goldsmith Donald W

Gong Shumo

Gonzalez Alejandro

Goobar Ariel M

Goret Philippe

Gosset Eric

Gottloeber Stefan

Gouda Naoteru

Govinder Keshlan S

Goyal Ashok Kumar

Gray Richard O
Gregory Stephen Albert

Greyber Howard D

Grishchuk Leonid P

Gudmundsson Einar $\mathrm{H}$

Gunn James E

Guzzo Luigi

Hagen Hans-Juergen

Hamilton Andrew J S

Hannestad Steen

Hardy Eduardo

Harms Richard James

Harrison Edward R

Hawking Stephen W

Hayashi Chushiro

He Xiang-Tao

Heavens Alan

Hellaby Charles William

Heller Michael

Henriksen Mark Jeffrey

Hewett Paul

Hewitt Adelaide

Hnatyk Bohdan

Hoyle Fred

Hu Esther M

Huchra John Peter

Hudson Michael J

Hwang Jai-chan

Icke Vincent

Ikeuchi Satoru

Impey Christopher D

Iovino Angela

Ishihara Hideki

Iyer B R

Jannuzi Buell Tomasson

Jaroszynski Michal

Jauncey David L

Jedamzik Karsten

Jetzer Philippe F

Jiang Shuding

Jones Bernard J T

Jones Laurence $\mathrm{R}$

Joshi Mohan N

Junkkarinen Vesa T

Juszkiewicz Roman

Kajino Toshitaka

Kandrup Henry Emil

Kang Hyesung

Kapoor Ramesh Chander

Karachentsev Igor D

Kasper U
Kato Shoji

Kaul Chaman

Kawabata Kinaki

Kawabata Kiyoshi

Kawasaki Masahiro

Kellermann Kenneth I

Kembhavi Ajit K

Khare Pushpa

Khmil Sergiy V

Kim Jik Su

King Lindsay $\mathbf{J}$

Kirilova Daniela

Kneib Jean-Paul

Kodama Hideo

Kokkotas Konstantinos

Kolb Edward W

Kompaneets Dmitriy A

Kormendy John

Kovetz Attay

Kozai Yoshihide

Kozlovsky B Z

Krasinski Andrzej

Kriss Gerard A

Kudrya Yury M

Kunth Daniel

Lacey Cedric

Lachieze-Rey Marc

Lahav Ofer

Lake Kayll William

Larionov Mikhael G

Lasota Jean-Pierre

Lausberg Andre

Layzer David

Lequeux James

Li Xiaoqing

Liddle Andrew

Liebscher Dierck-E

Lilje Per Vidar Barth

Lima Jose A S

Liu Yongzhen

Longair Malcolm S

Lonsdale Carol J

Lopez-Corredoira Martin

Loveday Johnathan $\mathrm{N}$

Lu Tan

Lucchin Francesco

Lukash Vladimir $\mathrm{N}$

Luminet Jean-Pierre

Lynden-Bell Donald

Maccagni Dario 


\begin{tabular}{|c|c|c|}
\hline MacCallum Malcolm A H & Novikov Igor D & Robinson I \\
\hline Maddox Stephen & Novosyadlyj Bohdan & Rocca-Volmerange Brigitte \\
\hline Maeda Kei-ichi & Novotny Jan & Roettgering Huub \\
\hline Maharaj Sunil Dutt & O'Connell Robert West & Romer Anita K \\
\hline Maia Marcio R G S & Oemler Jr Augustus & Rosquist Kjell \\
\hline Mamon Gary A & Okoye Samuel E & Rowan-Robinson Michael \\
\hline Mandolesi Nazzareno & Oliver Sebastian J & Roxburgh Ian W \\
\hline Mansouri Reza & Olowin Ronald Paul & Rubin Vera C \\
\hline Mao Shude & Omnes Roland & Rudnick Lawrence \\
\hline Marano Bruno & Onuora Lesley Irene & Rudnicki Konrad \\
\hline Mardirossian Fabio & Oscoz Alejandro & Ruffini Remo \\
\hline Marek John & Ott Heinz-Albert & Saar Enn \\
\hline Martinez-Gonzalez E & Oukbir Jamila & Sadat Rachida \\
\hline Mather John Cromwell & Ozernoy Leonid M & Sahni Varun \\
\hline Mathez Guy & Ozsvath I & Salvador-Sole Eduardo \\
\hline Matsumoto Toshio & Pachner Jaroslav & Salzer John Joseph \\
\hline Matzner Richard A & Padmanabhan $\mathrm{T}$ & Sanz Jose L \\
\hline Mavrides Stamatia & Padrielli Lucia & Sapar Arved \\
\hline Mellier Yannick & Page Don Nelson & Sargent Wallace L W \\
\hline Melott Adrian L & Pan Rong-Shi & Sasaki Misao \\
\hline Merighi Roberto & Parnovsky Sergei & Sasaki Shin \\
\hline Meszaros Attila & Partridge Robert B & Sato Humitaka \\
\hline Meszaros Peter & Pecker Jean-Claude & Sato Katsuhiko \\
\hline Meyer David M & Peebles P James E & Sato Shinji \\
\hline Meylan Georges & Pello Roser Descayre & Savage Ann \\
\hline Mezzetti Marino & Penzias Arno A & Sazhin Michail \\
\hline Misner Charles W & Perryman Michael A C & Schatzman Evry \\
\hline Miyoshi Shigeru & Persides Sotirios C & Schechter Paul L \\
\hline Mo Houjun & Peterson Bruce A & Scheuer Peter A G \\
\hline Mohr Joseph J & Petitjean Patrick & Schindler Sabine \\
\hline Moreau Olivier & Petrosian Vahe & Schmalzing Jens \\
\hline Morrison Philip & Plionis Manolis & Schmidt Brian P \\
\hline Moscardini Lauro & Popescu Nedelia A & Schmidt Maarten \\
\hline Mosconi Mirta B & Premadi Premana W & Schneider Donald P \\
\hline Muecket Jan P & Press William $\mathrm{H}$ & Schneider Jean \\
\hline Muller Richard A & Puget Jean-Loup & Schneider Peter \\
\hline Murakami Izumi & Qin Bo & Schramm Thomas \\
\hline Nambu Yasusada & Qu Qinyue & Schuch Nelson Jorge \\
\hline Narasimha Delampady & Ramella Massimo & Schucking Engelbert L \\
\hline Narlikar Jayant V & Rawlings Steven & Schuecker Peter \\
\hline Neeman Yuval & Raychaudhuri Amalkumar & Schultz G V \\
\hline Neves de Araujo Jose Carlos & Rebolo Rafael & Schumacher Gerard \\
\hline Nicoll Jeffrey Fancher & Rees Martin J & Scott Douglas \\
\hline Nishida Minoru & Reeves Hubert & Seiden Philip E \\
\hline Noerdlinger Peter D & Refsdal Sjur & Seielstad George A \\
\hline Noh Hyerim & Riazi Nematollah & Setti Giancarlo \\
\hline Noonan Thomas W & Ribeiro Marcelo B & Shandarin Sergei F \\
\hline Norman Colin A & Rindler Wolfgang & Shanks Thomas \\
\hline Nottale Laurent & Rivolo Arthur Rex & Shao Zhengyi \\
\hline Novello Mario & Roberts David Hall & Shaver Peter A \\
\hline
\end{tabular}




Shaviv Giora
Shaya Edward J
Shibata Masaru
Shivanandan Kandiah
Signore Monique
Silk Joseph I
Simon Rene L E
Sironi Giorgio
Sistero Roberto F
Smette Alain
Smith Rodney M
Smith Jr Harding E
Smoot III George F
Sokolowski Lech
Song Doo Jong
Souriau Jean-Marie
Spyrou Nicolaos
Srianand Roghunathan
Stecker Floyd W
Steigman Gary
Stewart John Malcolm
Stoeger William R
Straumann Norbert
Struble Mitchell F
Strukov Igor A
Subrahmanya C R
Suginohara Tatsushi
Sugiyama Naoshi
Sunyaev Rashid A
Surdej Jean M G
Sutherland William
Tagoshi Hideyuki
Takahara Fumio
Tammann Gustav Andreas
Tanabe Kenji
Tarter Jill C

$\begin{array}{ll}\text { Taruya Atsushi } & \text { Webster Adrian S } \\ \text { Thuan Trinh Xuan } & \text { Weinberg Steven } \\ \text { Tifft William G } & \text { Wesson Paul S } \\ \text { Tipler Frank Jennings } & \text { West Michael } \\ \text { Tomimatsu Akira } & \text { Wheeler John A } \\ \text { Tomita Kenji } & \text { White Simon David Manion } \\ \text { Tonry John } & \text { Whitrow Gerald James } \\ \text { Totani Tomonori } & \text { Wilkinson David T } \\ \text { Treder H J } & \text { Will Clifford M } \\ \text { Tremaine Scott Duncan } & \text { Wilson Albert G } \\ \text { Trevese Dario } & \text { Wilson Andrew S } \\ \text { Trimble Virginia L } & \text { Windhorst Rogier A } \\ \text { Tsamparlis Michael } & \text { Wolfe Arthur M } \\ \text { Tully Richard Brent } & \text { Woltjer Lodewijk } \\ \text { Turner Edwin L } & \text { Woszczyna Andrzej } \\ \text { Turner Michael S } & \text { Wright Edward L } \\ \text { Turnshek David A } & \text { Wu Xiangping } \\ \text { Tyson John Anthony } & \text { Xiang Shouping } \\ \text { Tytler David } & \text { Xu Chongming } \\ \text { Umemura Masayuki } & \text { Yang Lantian } \\ \text { Uson Juan M } & \text { Yokoyama Jun-ichi } \\ \text { Vagnetti Fausto } & \text { Yoshii Yuzuru } \\ \text { Vaidya P C } & \text { Yoshimura Motohiko } \\ \text { Valls-Gabaud David } & \text { Yushchenko Alexander V } \\ \text { van der Laan Harry } & \text { Zamorani Giovanni } \\ \text { van Haarlem Michiel } & \text { Zhang Jialu } \\ \text { Vedel Henrik } & \text { Zhang Tong-jie } \\ \text { Vettolani Giampaolo } & \text { Zhang Zhen-Jiu } \\ \text { Vishniac Ethan T } & \text { Zhou Youyuan } \\ \text { Vishveshwara C V } & \text { Zhu Shichang } \\ \text { Voglis Nikos } & \text { Zhu Xingfeng } \\ \text { von Borzeszkowski H H } & \text { Zhu Zong-Hong } \\ \text { Wagoner Robert V } & \text { Zieba Stanislaw } \\ \text { Wainwright John } & \text { Zou Zhenlong } \\ \text { Wambsganss Joachim } & \text { Zucca Elena } \\ \text { Wanas Mamdouh Ishaac } & \text { Zuiderwijk Edwardus J } \\ & \end{array}$




\section{Composition of Commission 49}

\section{Interplanetary Plasma \& Heliosphere/Plasma interplanétaire \& héliosphère}

$\begin{array}{ll}\text { PRESIDENT: } & \text { Vandas Marek } \\ \text { VICE-PRESIDENT: } & \text { Webb David F }\end{array}$

ORGANIZING COMMITTEE:

Bougeret Jean-Louis

Buti Bimla

Cramer Neil F

MEMBERS:

Ahluwalia Harjit Singh

Ananthakrishnan S

Anderson Kinsey A

Barnes Aaron

Barrow Colin $\mathrm{H}$

Barth Charles A

Benz Arnold O

Bertaux Jean-Loup

Blackwell Donald E

Blandford Roger David

Blum Peter

Bochsler Peter

Bonnet Roger $\mathrm{M}$

Brandt John C

Buechner Joerg

Burlaga Leonard $F$

Chamberlain Joseph $\mathrm{W}$

Chashei Igor V

Chassefiere Eric

Chen Biao

Chitre Dattakumar M

Chitre Shashikumar M

Chou Chih-Kang

Couturier Pierre

Cuperman Sami

Daglis Ioannis A

de Jager Cornelis

De Keyser Johan

Dinulescu Simona

Dolginov Arkady Z

Duldig Marcus Leslie

Durney Bernard

Dyson John E

Ergma E V

Eshleman Von R
Dryer Murray

Hollweg Joseph V

Kojima Masayoshi

Eviatar Aharon

Fahr Hans Joerg

Feynman Joan

Fichtner Horst

Field George B

Galvin Antoinette B

Goldman Martin V

Gosling John T

Grzedzielski Stanislaw Pr

Habbal Shadia Rifai

Harvey Christopher C

Heras Ana M

Heynderickx Daniel

Heyvaerts Jean

Holzer Thomas Edward

Huber Martin C E

Humble John Edmund

Inagaki Shogo

Ivanov Evgeny I

Jokipii J R

Joselyn Jo Ann c

Kakinuma Takakiyo T

Keller Horst Uwe

Lafon Jean-Pierre $\mathbf{J}$

Lai Sebastiana

Levy Eugene $\mathrm{H}$

Lotova Natalia A

Luest Reimar

Lundstedt Henrik

MacQueen Robert M

Mangeney Andre

Manoharan P K

Mason Glenn $\mathrm{M}$

Matsuura Oscar T

Mavromichalaki Helen
Sanahuja Blas

Verheest Frank

Meister Claudia Veronika

Melrose Donald B

Mendis Devamitta Asoka

Mestel Leon

Michel F Curtis

Moussas Xenophon

Nakagawa Yoshinari

Paresce Francesco

Parhi Shyamsundar

Parker Eugene N

Perkins Francis W

Pflug Klaus

Pneuman Gerald W

Polygiannakis John

Quemerais Eric

Raadu Michael A

Readhead Anthony C S

Reay Newrick K

Rickett Barnaby James

Riddle Anthony C

Ripken Hartmut W

Rosner Robert

Roth Michel A

Roxburgh Ian W

Rucinski Daniel

Russell Christopher T

Sagdeev Roald Z

Sarris Emmanuel T

Sastri Hanumath J

Sawyer Constance B

Schatzman Evry

Scherb Frank

Schindler Karl

Schmidt H U

Schreiber Roman 
Schwartz Steven Jay

Setti Giancarlo

Shea Margaret A

Smith Dean F

Sonett Charles $P$

Stone R G

Sturrock Peter A
Suess Steven T

Tritakis Basil P

Vainstein L A

van Allen James A

Vinod S Krishan

von Steiger Rudolf

Vucetich Hector
Wang Shunde

Wang Yi-ming

Watanabe Takashi

Weller Charles S

Wild John Paul

Wu Shi Tsan

Yeh Tyan 


\section{Composition of Commission 50}

Protection of Existing \& Potential Observatory Sites Protection des sites d'observatoires existants \& potentiels

PRESIDENT: Cohen Raymond J

VICE-PRESIDENT: Smith Malcolm G

ORGANIZING COMMITTEE:

Crawford David L Jiang Shi-Yang

Hidayat Bambang Malin David F

McNally Derek

Isobe Syuzo

Spoelstra T A Th

\section{MEMBERS:}

Ardeberg Arne L

Arsenijevic Jelisaveta

Barreto Luiz Muniz

Benkhaldoun Zouhair

Bensammar Slimane

Bhattacharyya J C

Blanco Carlo

Blanco Victor $M$

Brown Robert Hamilton

Burstein David

Cayrel Roger

Cinzano Pierantonio

Costero Rafael

Coyne George V

Davis Donald $\mathrm{R}$

Davis John

Dawe John Alan

de Greiff J Arias

Dommanget $\mathrm{J}$

Gibson David Michael

Pankonin Vernon Lee

Goebel Ernst

Heck Andre

Helmer Leif

Ilyasov Sabit

Kadiri Samir

Pound Marc W

Sanchez Francisco

Schilizzi Richard T

Shcheglov P V

Kahlmann Hans Cornelis

Shetrone Matthew C

Kontizas Evangelos

Smith Francis Graham

Kontizas Mary

Sullivan Woodruff $T$

Kovalevsky Jean

Suntzeff Nicholas B

Torres Carlos

Kozai Yoshihide

Leibowitz Elia M

Mahra H S

Markkanen Tapio

Mattig W

Menzies John W

Mitton Jacqueline

Murdin Paul G

Nelson Burt

Dunkelman Lawrence

Galan Maximino J

Oezel Mehmet Emin

Osorio Jose J S P

Gergely Tomas Esteban

Owen Frazer Nelson

Torres Carlos Alberto

Tremko Jozef

Upgren Arthur R

van den Bergh Sidney

Vetesnik Miroslav

Walker Merle F

Whiteoak John B

Woolf Neville J

Woszczyk Andrzej

Wu Mingchan

Yano Hajime

Ziznovsky Jozef 


\title{
Composition of Commission 51
}

Bioastronomy: Search for Extraterrestrial Life

Bioastronomie: recherche de la vie extraterrestre

\author{
PRESIDENT: Bowyer C Stuart
}

VICE-PRESIDENT: Meech Karen

ORGANIZING COMMITTEE:

$\begin{array}{ll}\text { Almar Ivan } & \text { Mayor Michel } \\ \text { Colomb Fernando R } & \text { Owen Tobias C } \\ \text { Drake Frank D } & \text { Sullivan Woodruff T } \\ \text { Leger Alain } & \text { Tarter Jill C }\end{array}$

Wellington Kelvin

Wilson Thomas L

MEMBERS:

Al-Naimiy Hamid M K

Couper Heather Miss

Gehrels Tom

Al-sabti Abdul Adim

Ando Hiroyasu

Balazs Bela A

Ball John A

Bania Thomas Michael

Currie Douglas G

Daigne Gerard

Davis Michael M

Dawe John Alan

de Graaff W

Barbieri Cesare

de Jager Cornelis

Basu Baidyanath

Basu Dipak

Baum William A

Beaudet Gilles

Beckman John E

Beckwith Steven V W

Beebe Reta Faye

Benest Daniel

Berendzen Richard

Bernacca Pietio L

Billingham John

Biraud Francois

Bless Robert C

Boyce Peter B

Bracewell Ronald N

Broderick John

Brown Ronald D

Burke Bernard $\mathrm{F}$

Calvin William $\mathrm{H}$

Campusano Luis E

de Jonge $\mathbf{J} \mathrm{K}$

de Loore Camiel

de Vincenzi Donald

Delsemme Armand $\mathrm{H}$

Dick Steven J

Dixon Robert S

Dorschner Johann

Doubinskij Boris A

Downs George S

Dyson Freeman J

Eccles Michael J

Ellis George F R

Epstein Eugene E

Evans Neal J

Fazio Giovanni G

Fejes Istvan

Feldman Paul A

Field George B

Firneis Friedrich J

Firneis Maria G

Fisher Philip C

Fredrick Laurence W

Ghigo Francis D

Ginzburg Vitaly L

Giovannelli Franco

Godoli Giovanni

Golden Aaron

Goldsmith Donald W

Gott J Richard

Goudis Christos D

Greenberg J Mayo

Greenstein Jesse L

Gregory Philip C

Gulkis Samuel

Gunn James E

Gurm Hardev S

Haddock Fred T

Haisch Bernhard Michael

Hajduk Anton

Hale Alan

Harrison Edward R

Hart Michael $\mathrm{H}$

Heck Andre

Heeschen David S

Herczeg Tibor J

Hershey John L

Heudier Jean-Louis

Hinners Noel W

Hirabayashi Hisashi

Carr Thomas D

Chaisson Eric J

Chou Kyong Chol

Freire Ferrero Rubens G

Hoang Binh Dy

Fujimoto Masa-Katsu

Hoegbom Jan A

Hollis Jan Michael

Fujimoto Mitsuaki

Horowitz Paul

Clark Thomas A

Gatewood George

Hunten Donald M 
Hunter James H

Hysom Edmund J

Idlis Grigorij M

Irvine William $M$

Israel Frank $P$

Jastrow Robert

Jeffers Stanley

Jennison Roger C

Jones Eric M

Jugaku Jun

Kafatos Minas

Kapisinsky Igor

Kardashev Nicolay S

Kaufmann Pierre

Keay Colin S 1

Keller Hans Ulrich

Kellermann Kenneth I

Klein Michael J

Knowles Stephen $\mathrm{H}$

Kocer Durcun

Koch Robert $\mathrm{H}$

Koeberl Christian

Kraus John D

Ksanfomaliti Leonid V

Kuiper Thomas B H

Kuzmin Arkadii D

Lafon Jean-Pierre J

Laques Pierre

Lee Sang Gak

Levasseur-Regourd A.-C.

Lilley Edward A

Lippincott Sarah Lee

Loden Lars Olof

Lovell Sir Bernard

Maffei Paolo

Margrave Jr Thomas Ewing

Marov Mikhail Ya

Martin Anthony R

Martin Maria Cristina

Marx Gyorgy

Matsakis Demetrios N

Matsuda Takuya

Matthews Clifford

Mavridis L N

McAlister Harold A

McDonough Thomas $\mathrm{R}$

Mendoza V Eugenio E

Milet Bernard L

Minn Young Key

Mirabel Igor Felix
Morimoto Masaki

Moroz Vasilis I

Morris Mark Root

Morrison David

Morrison Philip

Muller Richard A

Nakagawa Yoshinari

Nelson Robert M

Niarchos Panayiotis

Norris Raymond Paul

Oda Minoru

Ollongren A

Ostriker Jeremiah $\mathbf{P}$

Pacini Franco

Papagiannis Michael D

Parijskij Yuri N

Pasinetti Laura E

Perek Lubos

Ponsonby John E B

Prochazka Franz V

Qiu Puzhang

Qiu Yaohui

Quintana Hernan

Quintana Jose M

Quirrenbach Andreas

Raghavan Nirupama

Rajamohan R

Reay Newrick K

Rees Martin J

Riihimaa Jorma J

Robinson Leif J

Rodriguez Luis F

Rood Robert T

Rowan-Robinson Michael

Rubin Robert Howard

Russell Jane L

Sakurai Kunitomo

Sancisi Renzo

Scargle Jeffrey D

Schatzman Evry

Schild Rudolph E

Schneider Jean

Schober Hans J

Schuch Nelson Jorge

Seeger Charles Louis III

Seielstad George A

Seiradakis John Hugh

Shapiro Maurice M

Shen Chun-Shan

Shimizu Mikio
Shostak G Seth

Singh $\mathrm{H} \mathrm{P}$

Sivaram C

Slysh Viacheslav I

Snyder Lewis E

Sofue Yoshiaki

Stalio Roberto

Stein John William

Straizys V

Sturrock Peter A

Takaba Hiroshi

Takada-Hidai Masahide

Tavakol Reza

Tedesco Edward F

Tejfel Viktor G

Terzian Yervant

Thaddeus Patrick

Tolbert Charles R

Toro Tibor

Tovmassian Hrant M

Townes Charles Hard

Trimble Virginia L

Turner Edwin L

Turner Kenneth C

Valbousquet Armand

Vallee Jacques $P$

Valtaoja Leena

Van Flandern Tom

Varshalovich Dimitrij

Vauclair Gerard P

Vazquez Manuel

Venugopal V R

Verschuur Gerrit L

Vogt Nikolaus

von Hoerner Sebastian

Wallis Max K

Watson Frederick Garnett

Welch William J

Wesson Paul S

Wetherill George W

Wielebinski Richard

Williams Iwan $\mathbf{P}$

Willson Robert Frederick

Wolstencroft Ramon D

Wright Alan E

Wright Ian $P$

Ye Shuhua

Zuckerman Ben M 